UNIVERSIDADE DE SÃO PAULO

INSTITUTO DE GEOCIÊNCIAS

\title{
AVALIAÇÃO DO POTENCIAL DE GERAÇÃO DE METANO E DIÓXIDO DE CARBONO BIOGÊNICOS EM FOLHELHOS DO SUDESTE BRASILEIRO
}

Dailson José Bertassoli Junior

Orientador: André Oliveira Sawakuchi

DISSERTAÇÃO DE MESTRADO

Programa de Pós-Graduação em Geoquímica e Geotectônica

São Paulo

2016 
Autorizo a reprodução e divulgação total ou parcial deste trabalho, por qualquer meio convencional ou eletrônico, para fins de estudo e pesquisa, desde que citada a fonte.

Ficha catalográfica preparada pelo Serviço de Biblioteca e Documentação do Instituto de Geociências da Universidade de São Paulo

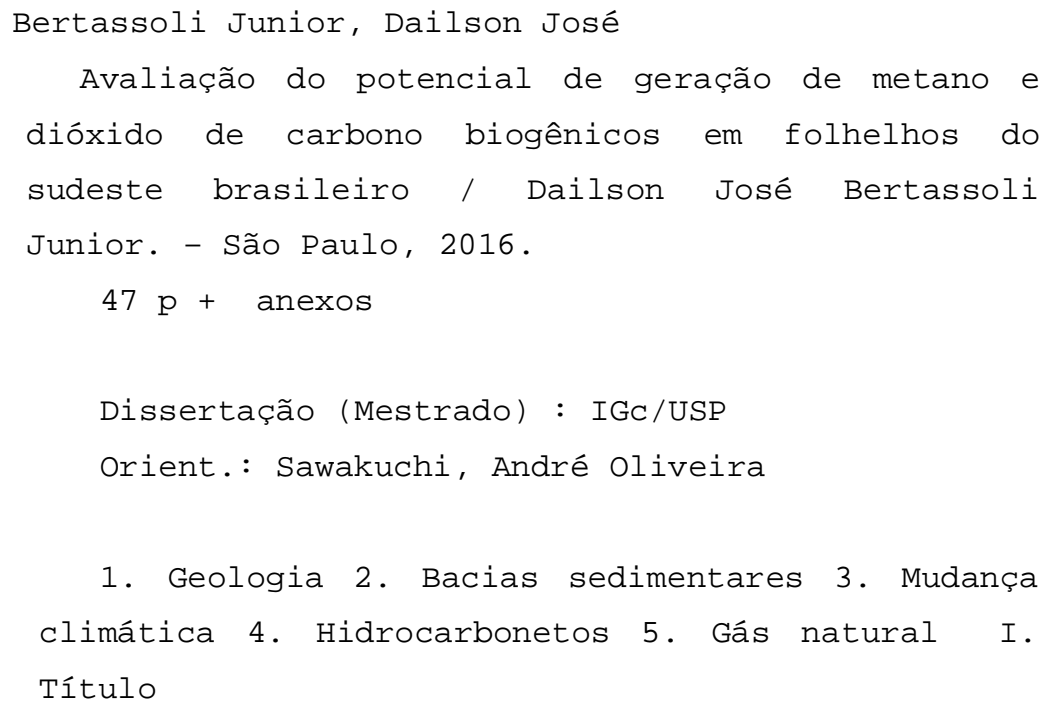


In Memoriam

Dailson José Bertassoli (1960-2015) 


\section{AGRADECIMENTOS}

Agradeço primeiramente ao Prof. Dr. André Oliveira Sawakuchi pelas ideias, oportunidades, amizade e orientação ao longo dos últimos anos, além de sua conduta exemplar como cientista.

Ao Dr. Henrique Oliveira Sawakuchi pela paciência e dedicação durante todo o projeto. Sua colaboração foi fundamental para o sucesso deste trabalho.

Aos responsáveis e técnicos dos Laboratórios de Análise Ambiental e Geoprocessamento (CENA-USP) e do Laboratório de Espectrometria Gama e Luminescência (IGC-USP), Prof. Dr. Alex Krusche, Alexandra, Thays e Luciana, por todo o apoio prestado.

Ao Prof. Dr. Sergio Brochsztain, à Bruna Castanheira e à UFABC pelo enorme auxílio na realização das análises de adsorção de nitrogênio.

Aos colegas Marcelo, Nazaré, Victor, Maria e Laura pela amizade, paciência e pelo apoio material e imaterial ao longo de todo o trabalho.

Ao meu irmão Marcelo e à minha namorada Amanda, à minha mãe Mirian e ao meu padrasto Eduardo, ao meu pai Dailson e à minha madrasta Daniela, meus sinceros agradecimentos por alegrarem todos os dias da minha vida.

E aos amigos de Mogi e da Turma 51 pela parceria ao longo de tantos anos. 
"O aspecto mais triste da vida atualmente é que a ciência adquire conhecimento mais rápido do que a sociedade adquire sabedoria."

Isaac Asimov (1920-1992) 


\section{SUMÁRIO}

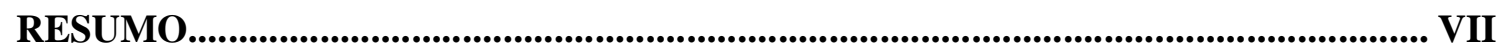

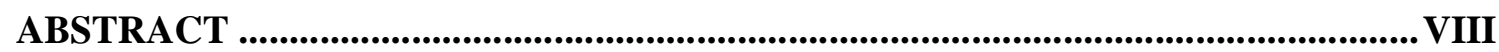

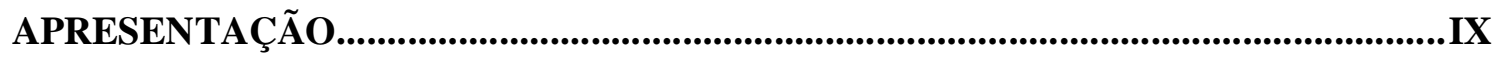

1 INTRODUÇÃ $O$......................................................................................................... 10

2 ORIGEM DO METANO BIOGÊNICO ..............................................................12

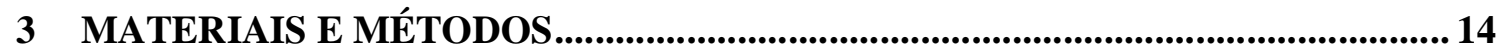

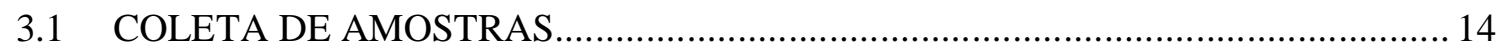

3.2 ANÁLISE DO TEOR DE CARBONO ORGÂNICO TOTAL ...................................... 16

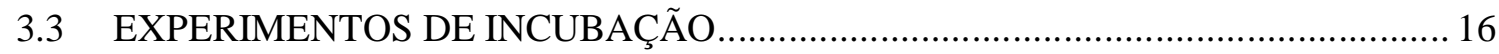

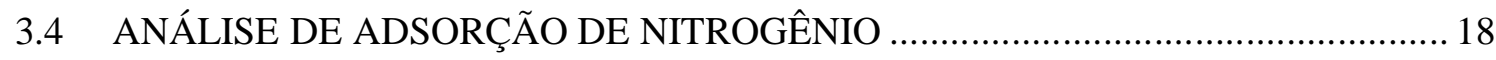

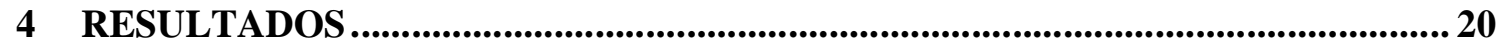

4.1 QUANTIFICAÇÃO DE COT E PRODUÇÃO POTENCIAL DE CH $\mathrm{E} \mathrm{CO}_{2} \ldots \ldots \ldots \ldots . . .20$

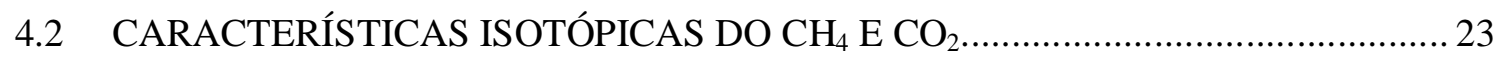

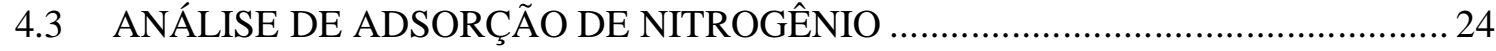

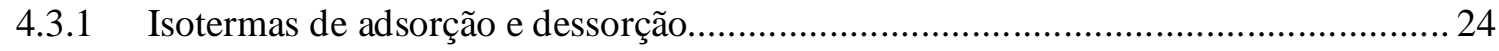

4.3.2 Superfície específica e volume de poro.................................................................. 26

4.3.3 Distribuição do tamanho de poro: Métodos BJH e DFT.............................................. 29

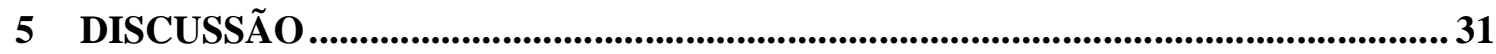

5.1 FATORES CONTROLADORES DA GERAÇÃO DE $\mathrm{CH}_{4} \mathrm{E} \mathrm{CO}_{2} \ldots \ldots \ldots \ldots \ldots \ldots \ldots \ldots \ldots \ldots . . . . . . . . . . . . . .11$

5.2 POTENCIAL PARA PRODUÇÃO DE GÁS NATURAL E CONTRIBUIÇÃO

PARA EMISSÃO DE GASES DO EFEITO ESTUFA …………………….............. 34

5.3 INFLUÊNCIA DA ESTRUTURA DE POROS PARA A GERAÇÃO DE $\mathrm{CH}_{4} \ldots \ldots \ldots . . . .36$

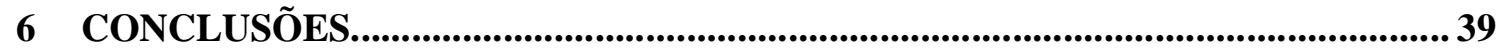

7 REFERÊNCIAS BIBLIOGRÁFICAS................................................................40

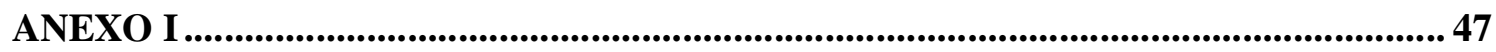




\section{RESUMO}

Sistemas não-convencionais de metano biogênico em folhelhos podem representar importante recurso energético e contribuir significativamente para emissões de gases do efeito estufa. Com o intuito de avançar na compreensão dos controles na geração de metano $\left(\mathrm{CH}_{4}\right)$ e dióxido de carbono $\left(\mathrm{CO}_{2}\right)$ em folhelhos ricos em matéria orgânica, o presente estudo avaliou o potencial de geração e a estrutura de poros de folhelhos das bacias sedimentares do Paraná e Taubaté, localizadas na região sudeste do Brasil. As formações Ponta Grossa (Devoniano, Bacia do Paraná), Irati (Permiano, Bacia do Paraná) e Tremembé (Paleógeno, Bacia de Taubaté) foram analisadas de modo a obter-se taxas de produção biogênica de $\mathrm{CH}_{4}$ e $\mathrm{CO}_{2}$ sob diferentes condições. Para tanto, foram efetuadas incubações de 24 amostras de folhelho em laboratório, sob meios seco, aquoso e com adição de ácido ácetico, durante períodos de até 1 ano. Também foram realizadas análises para a determinação do teor de carbono orgânico e testes de adsorção para caracterização de poros e superfície específica das amostras de folhelho com o intuito de compreender o papel destas variantes na geração de gases biogênicos. As taxas de produção de gás biogênico em amostras incubadas a seco atingiram

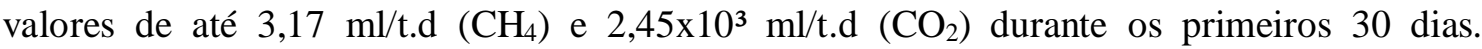
Amostras incubadas com adição de água demonstraram aumento de $54 \%$ na produção de $\mathrm{CH}_{4}$ e $151 \%$ na produção de $\mathrm{CO}_{2}$. A adição de ácido acético no sistema foi responsável pelo reínicio ou aumento da produção de $\mathrm{CH}_{4}$ e $\mathrm{CO}_{2}$ na maioria dos casos avaliados. A Formação Irati apresentou o maior potencial para produção de metano biogênico entre as unidades estratigráficas estudadas, fator que pode estar ligado à biodegradação de petróleo pesado presente nos poros. O volume total de poros e a superfície específica de amostras aparenta não afetar a produção biogênica. Entretanto, a umidade e disponibilidade de substrato exercem controle predominante no potencial de geração de $\mathrm{CH}_{4}$ e $\mathrm{CO}_{2}$ biogênicos em folhelhos ricos em matéria orgânica.

Palavras-chave: Gás de folhelho; Bacia do Paraná; Bacia de Taubaté; Metanogênese; Emissões geológicas de metano e dióxido de carbono; Mudanças climáticas. 


\begin{abstract}
Unconventional biogenic shale gas systems may represent an important energy resource and significantly contribute to geological greenhouse gases emissions. In order to better understand the controls on biogenic methane $\left(\mathrm{CH}_{4}\right)$ and carbon dioxide $\left(\mathrm{CO}_{2}\right)$ generation in organic-rich shales, the present study evaluated the generation potential and the pore structure of shales from Taubaté and Paraná basins, located in southeastern Brazil. The Ponta Grossa (Devonian, Paraná basin), Irati (Permian, Paraná basin) and Tremembé Formations were analyzed in order to quantify production rates of biogenic $\mathrm{CH}_{4}$ and $\mathrm{CO}_{2}$ under distinct experimental conditions. Twenty four shale samples were used for batch incubations under dry, wet and acetic acid solution conditions during time periods reaching up to 1 year. The organic carbon content and nitrogen adsorption analysis for determining specific surface area and porosity were also performed to evaluate their role on biogenic gas generation.The biogenic gas production rates in samples under dry conditions reached up to $3.17 \mathrm{ml} / \mathrm{t} . \mathrm{d}\left(\mathrm{CH}_{4}\right)$ and $2.45 \times 10^{3} \mathrm{ml} / \mathrm{t} . \mathrm{d}\left(\mathrm{CO}_{2}\right)$ during the first 30 days of incubation. Samples under wet conditions demonstrated production rates $54 \%$ higher for $\mathrm{CH}_{4}$ and $151 \%$ higher for $\mathrm{CO}_{2}$ in comparison with dry tests. Acetic acid addition restarted or increased $\mathrm{CH}_{4}$ and $\mathrm{CO}_{2}$ production in most cases. The Irati Formation showed the highest potential for biogenic methane production, which could be linked to the biodegradation of heavy liquid hydrocarbons occurring in this unit. Total pore volume and specific surface does not appear to significantly affect the biogenic production of $\mathrm{CH}_{4}$ and $\mathrm{CO}_{2}$. However, water content and substrate availability would exert predominant control over the biogenic gas generation within organic rich shales.
\end{abstract}

Keywords: Shale gas; Paraná basin; Taubaté basin; Methanogenesis; Geologic emissions of greenhouse gases; Climate change. 


\begin{abstract}
APRESENTAÇÃO
Os resultados desta dissertação de mestrado são apresentados na forma de um manuscrito científico a ser submetido para publicação no periódico Organic Geochemistry. O manuscrito Biogenic methane and carbon dioxide generation in organic-rich shales from southeastern Brazil (Bertassoli et al.) resulta da dissertação de mestrado em questão. Tal artigo foi redigido em língua inglesa, consta no presente volume como anexo e sua formatação segue as diretrizes do periódico Organic Geochemistry.

Além do manuscrito anexo, esta dissertação de mestrado inclui capítulo introdutório, descrição dos materiais e métodos empregados, síntese dos resultados, discussões e conclusões do artigo em língua portuguesa.
\end{abstract}




\section{INTRODUÇÃO}

A busca pela soberania energética tem sido objeto de recorrentes debates internacionais desde 1973, quando o embargo aos EUA imposto pela Organização dos Países Exportadores de Petróleo (OPEP) teve significativo impacto econômico em escala global (ROEDER, 2005; WANG et al., 2014). A produção de gás natural nos EUA, que demonstrava novos sinais de declínio no início do século XXI, passou por uma fase de renovação tecnológica que favoreceu a reversão de tal panorama, uma vez que a combinação de técnicas de perfuração horizontal e fraturamento hidráulico permitiram o aproveitamento de reservas de gás natural em rochas de baixa permeabilidade, tais como folhelhos (KUUSKRAA et al., 2011).

Além de impactos nos cenários político e econômico, a substituição do carvão mineral por gás natural em termelétricas estadunidenses contribuiu para a queda de $7,7 \%$ na emissão de dióxido de carbono derivado de combustíveis fósseis entre 2006 e 2011 (WANG et al., 2014).

Embora os efeitos climáticos relacionados aos combustíveis fósseis realcem a necessidade da adoção de fontes energéticas com baixa emissão de carbono, diversos autores destacam que a grande lacuna ainda existente entre tais fontes de energia inviabiliza uma transição imediata, defendendo o gás natural como uma das principais opções para a redução das emissões de gases de efeito estufa em curto prazo (HOFFERT, 2010; MELIKOGLU, 2014; PACALA; SOCOLOW, 2004).

O melhor aproveitamento do gás natural como fonte energética no Brasil também reduziria diversas vulnerabilidades climáticas, sociais e ambientais associadas à implantação de usinas hidrelétricas, principal fonte de energia elétrica da matriz energética brasileira (ANSAR et al., 2014; DE FARIA et al., 2015; FEARNSIDE, 2014; SABAJ PÉREZ, 2015).

Ainda que a disposição geográfica irregular das reservas convencionais de gás natural dificulte o aproveitamento de tal recurso em maior escala, o investimento na exploração de reservas não-convencionais pode contribuir para a mitigação desta limitação (MELIKOGLU, 2014). Neste contexto, destaca-se o gás natural de origem biogênica, cujas reservas estimadas por Rice (1993) representariam mais de $20 \%$ dos recursos de gás natural globais.

Sistemas não-convencionais de gás biogênico são comumente negligenciados em campanhas exploratórias, fato associado tanto à falta de compreensão acerca de tais sistemas quanto às baixas taxas de retorno dos recursos em questão (SHURR; RIDGLEY, 2002). 
Entretanto, tais reservas constituem nova fronteira exploratória que pode suprir a demanda de gás natural em diversos países e contribuir favoravelmente com a balança comercial de países em desenvolvimento que importam combustíveis fósseis de grandes produtores (MELIKOGLU, 2014). Adicionalmente, por ocuparem porções mais rasas em bacias sedimentares, possuem custos reduzidos de perfuração de poços e maior facilidade no monitoramento de aquíferos adjacentes ao reservatório, os quais podem sofrer possíveis danos ambientais.

Apesar da ampla distribuição de folhelhos orgânicos nas bacias intracratônicas brasileiras e do grande número de trabalhos científicos visando à caracterização de possíveis reservatórios não-convencionais de metano em todo o mundo (OSBORN; MCINTOSH, 2010; SCHLEGEL et al., 2011; ZHANG et al., 2013), nota-se que poucos trabalhos sobre gás natural nas unidades brasileiras de folhelho foram publicados até o momento (EIA/ARI, 2013).

Destaca-se ainda a necessidade de trabalhos de caracterização do potencial de produção de gás biogênico em folhelhos brasileiros para avaliar a contribuição como fonte de gases de efeito estufa para a atmosfera, a partir de emissões geológicas de metano $\left(\mathrm{CH}_{4}\right)$ e dióxido de carbono $\left(\mathrm{CO}_{2}\right)$ (ETIOPE; KLUSMAN, 2002; ETIOPE, 2004).

Este trabalho avalia fatores condicionantes da geração biogênica de $\mathrm{CH}_{4}$ e $\mathrm{CO}_{2}$ em folhelhos das bacias do Paraná (formações Ponta Grossa e Irati) e Taubaté (Formação Tremembé), além de caracterizar a porosidade, superfície específica e teor de carbono destas unidades. O trabalho avalia o potencial de geração de metano e dióxido de carbono biogênicos a partir de incubações de amostras de folhelhos e da caracterização isotópica dos gases produzidos.

Portanto, esta dissertação de mestrado tem como objetivo a caracterização do potencial de produção e armazenamento natural de metano e dióxido de carbono biogênicos em folhelhos ricos em matéria orgânica das bacias do Paraná e Taubaté. Este trabalho também visa avançar no entendimento de fatores controladores do metabolismo de microorganismos metanogênicos, uma vez que compara o desenvolvimento de comunidades anaeróbicas atuantes na produção de metano e dióxido de carbono em folhelhos com diferentes propriedades texturais e composicionais, avaliadas sob condições laboratoriais similares. 


\section{ORIGEM DO METANO BIOGÊNICO}

Acumulações de metano em bacias sedimentares possuem duas possíveis origens: "biogênica", associada à produção de metano a partir do metabolismo de comunidades de micróbios metanogênicos, e "termogênica", gerada por reações térmicas de quebra de matéria orgânica fóssil (querogênio) (SCHOELL, 1980). Entretanto, reservas de gás natural podem possuir a mistura de ambos os tipos de metano (WHITICAR, 1999).

A produção de metano biogênico em subsuperfície está associada à Archeas metanogênicas e depende de condições ambientais específicas para sua ocorrência (KOTELNIKOVA, 2002). Condições anaeróbicas e ambientes com baixo teor de sulfato são os principais controles da metanogênese biológica, uma vez que Archeas atuam em ambiente anaeróbico e bactérias redutoras de sulfato se sobrepõem às metanogênicas na utilização de hidrogênio e acetato (OREMLAND; POLCIN, 1982).

A geração de metano biogênico também é controlada pela temperatura e pela disponibilidade de espaço e substrato (KOTELNIKOVA, 2002; ZINDER, 1993). Kotelnikova (2002) apresenta como fatores mais favoráveis à geração de metano temperatura entre $9{ }^{\circ} \mathrm{C} \mathrm{e}$ $11{ }^{\circ} \mathrm{C}$ e poros ou fraturas com pelo menos $1 \mu \mathrm{m}$ de diâmetro. Zeikus e Winfrey (1976), por sua vez, estabelecem temperatura entre $35{ }^{\circ} \mathrm{C}$ e $42{ }^{\circ} \mathrm{C}$ como condições ideais para o desenvolvimento de comunidades metanogênicas. O substrato deve ser preferencialmente rico em matéria orgânica, $\mathrm{CO}_{2}$ e hidrogênio $\left(\mathrm{H}_{2}\right)$, uma vez que tais compostos desempenham importantes papéis no metabolismo metanogênico (WHITICAR, 1999).

Conforme destacado por Rice (1993), o metano biogênico em subsuperfície é gerado principalmente pela redução de $\mathrm{CO}_{2}\left(\mathrm{CO}_{2}+4 \mathrm{H}_{2} \rightarrow \mathrm{CH}_{4}+2 \mathrm{H}_{2} \mathrm{O}\right)$ e pela fermentação de acetato $\left(\mathrm{CH}_{3} \mathrm{COOH} \rightarrow \mathrm{CH}_{4}+\mathrm{CO}_{2}\right)$. Entretanto, ácido metanoico, álcool, amidas metiladas e sulfetos também são utilizados como substrato por comunidades metanogênicas (ZINDER, 1993).

A assinatura isotópica do carbono $\left(\delta^{13} \mathrm{C}\right)$ é uma importante ferramenta para o reconhecimento da origem do metano (RICE, 1993; WHITICAR, 1999; WOLTERMATE; WHITICAR; SCHOELL, 1984). A presença de metano com $\delta^{13} \mathrm{C}_{\mathrm{CH} 4}$ mais leve que -50\%o e o predomínio do metano sobre outros alcanos ( $>98 \%$ de metano) são fatores comumente utilizados como indicadores de origem biogênica do gás natural (RICE; CLAYPOOL, 1981; RICE, 1993; WHITICAR, 1999). Entretanto, extrapolações isotópicas como esta devem ser consideradas com precaução, uma vez que fatores como temperatura e a composição do substrato podem alterar a assinatura isotópica obtida e sua interpretação (KOTELNIKOVA, 
2002; WHITICAR, 1999). Martini et al. (2003) destacam que a alta variabilidade de $\delta^{13} \mathrm{C}_{\mathrm{CH} 4}$ (-100 a -31\%o) observada em reservas de metano biogênico está associada às características do substrato precursor e do caminho metabólico utilizado.

O fracionamento isotópico é também influenciado por questões como maturidade térmica, disponibilidade de nutrientes e processos pós-geração, como migração e oxidação do $\mathrm{CH}_{4}$ (WHITICAR, 1999). Efeitos isotópicos do metabolismo microbial podem ser melhor entendidos a partir da avaliação dos fatores de fracionamento envolvidos em cada caso (KOTELNIKOVA, 2002).

Quanto à acumulação do metano gerado, ressalta-se que o potencial de folhelhos ricos em matéria orgânica como reservatório de gás depende não só de sua porosidade, que controla sua capacidade para estocar gás livre, mas também de seu potencial de adsorção (CURTIS et al., 2012). A adsorção de metano em folhelhos é controlada principalmente pelo conteúdo de matéria orgânica (MO). Compostos inorgânicos, por sua vez, apenas afetam o potencial de adsorção do folhelho quando baixas concentrações de MO ou quando grandes teores de esmectita estão presentes (GASPARIK et al., 2014). 


\section{MATERIAIS E MÉTODOS}

\subsection{COLETA DE AMOSTRAS}

As amostras de folhelho utilizadas neste trabalho foram coletadas em afloramentos das formações Irati e Ponta Grossa, na margem leste da Bacia do Paraná nos estados de São Paulo e Paraná, e em afloramentos da Formação Tremembé, na região central da Bacia de Taubaté no Estado de São Paulo (Figura 1). As 24 amostras selecionadas para estudo foram obtidas a partir de cortes de estrada, ferrovias e frentes de lavra em dois trabalhos de campo realizados nos meses de Maio e Agosto no ano de 2014 (Tabela 1 e Figura 2).

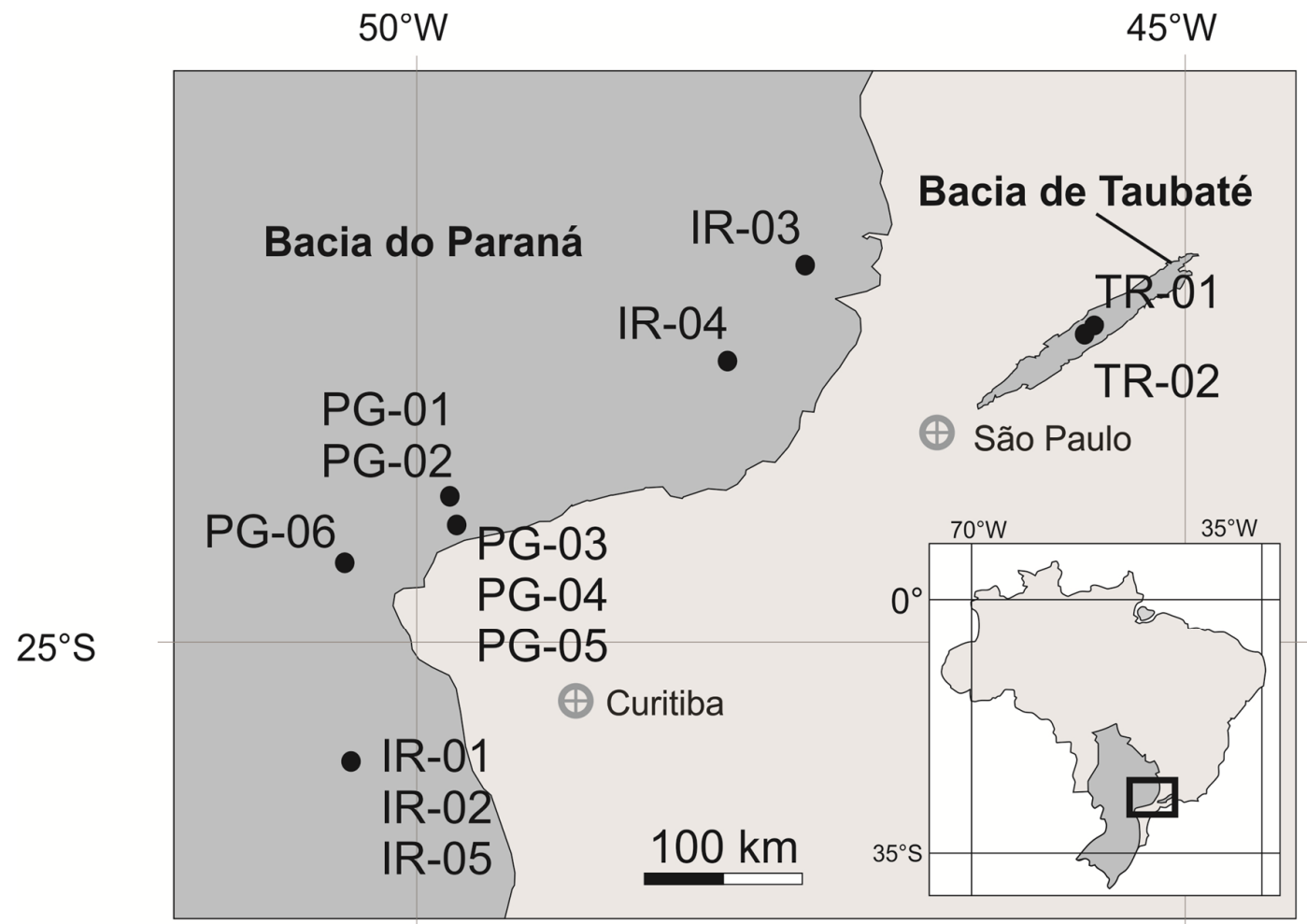

Figura 1 - Localização dos pontos de coleta de amostras das formações Irati (IR-01 a IR-05), Ponta Grossa (PG-01 a PG-06) e Tremenbé (TR-01 e TR-02). 

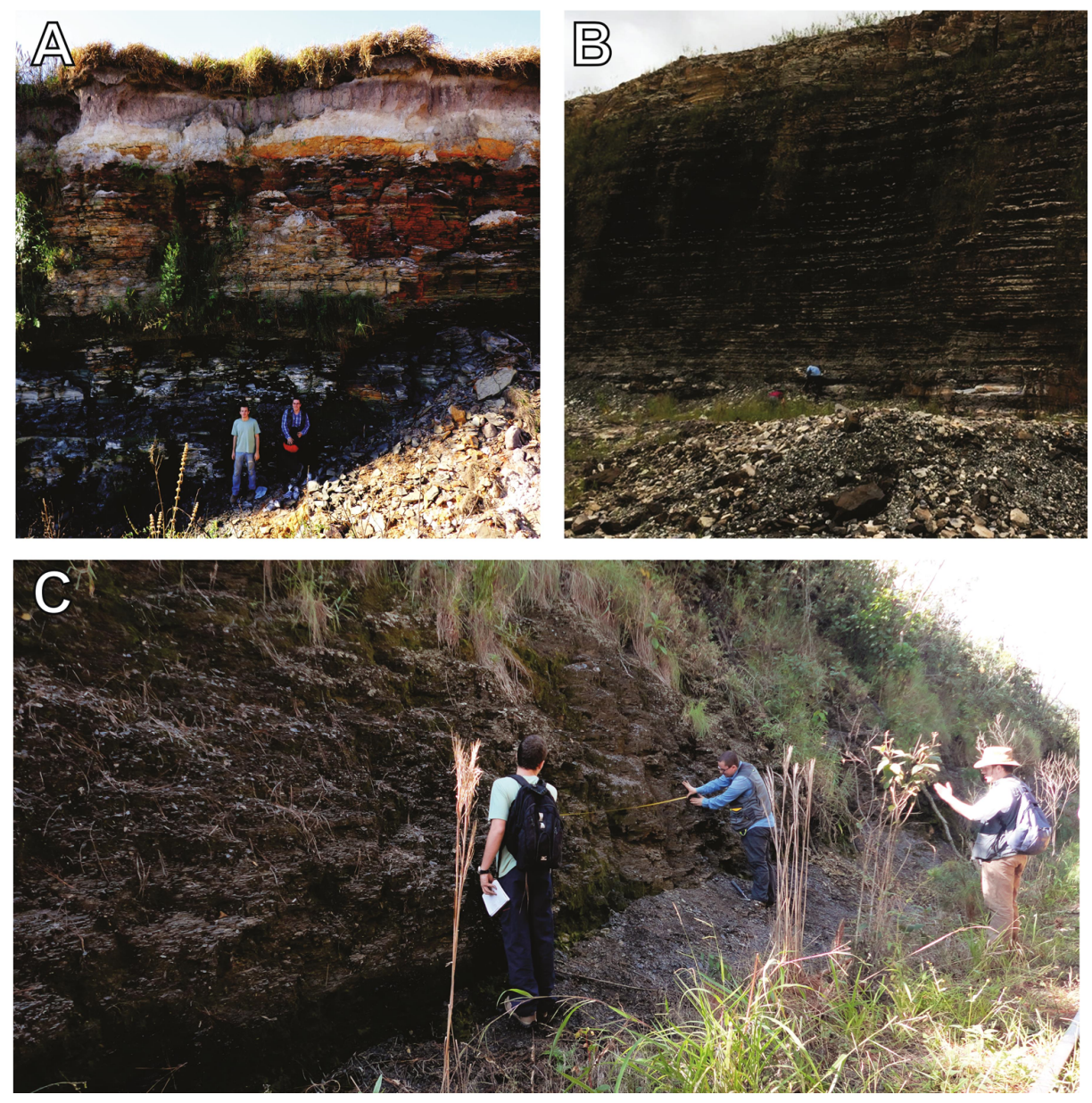

Figura 2 - Afloramentos das formações Tremembé (A), Irati (B) e Ponta Grossa (C) nas áreas estudadas.

Tabela 1 - Localização das amostras coletadas em afloramentos das formações Ponta Grossa (12 amostras), Irati (6 amostras) e Tremembé (6 amostras).

\begin{tabular}{|l|l|c|l|l|}
\hline Ponto & Formação & Número de amostras & Latitude & Longitude \\
\hline PG-01 & Ponta Grossa & 1 & $24^{\circ} 04^{\prime} 47^{\prime \prime S}$ & $49^{\circ} 46^{\prime} 30^{\prime \prime} \mathrm{W}$ \\
\hline PG-02 & Ponta Grossa & 1 & $24^{\circ} 04^{\prime} 48^{\prime \prime} \mathrm{S}$ & $49^{\circ} 46^{\prime} 24^{\prime \prime} \mathrm{W}$ \\
\hline PG-03 & Ponta Grossa & 4 & $24^{\circ} 14^{\prime} 30^{\prime \prime} \mathrm{S}$ & $49^{\circ} 43^{\prime} 55^{\prime \prime} \mathrm{W}$ \\
\hline PG-04 & Ponta Grossa & 1 & $24^{\circ} 14^{\prime} 24^{\prime \prime} \mathrm{S}$ & $49^{\circ} 43^{\prime} 57^{\prime \prime} \mathrm{W}$ \\
\hline PG-05 & Ponta Grossa & 1 & $24^{\circ} 14^{\prime} 24^{\prime \prime} \mathrm{S}$ & $49^{\circ} 43^{\prime} 57^{\prime \prime} \mathrm{W}$ \\
\hline PG-06 & Ponta Grossa & 4 & $24^{\circ} 30^{\prime} 03^{\prime \prime} \mathrm{S}$ & $50^{\circ} 26^{\prime} 45^{\prime \prime} \mathrm{W}$ \\
\hline IR-01 & Irati & 1 & $25^{\circ} 53^{\prime} 35^{\prime \prime} \mathrm{S}$ & $50^{\circ} 22^{\prime} 42^{\prime \prime} \mathrm{W}$ \\
\hline IR-02 & Irati & 1 & $25^{\circ} 49^{\prime} 34^{\prime \prime} \mathrm{S}$ & $50^{\circ} 24^{\prime} 52^{\prime \prime} \mathrm{W}$ \\
\hline IR-03 & Irati & 2 & $22^{\circ} 32^{\prime} 41^{\prime \prime} \mathrm{S}$ & $47^{\circ} 26^{\prime} 26^{\prime \prime} \mathrm{W}$ \\
\hline IR-04 & Irati & 1 & $23^{\circ} 10^{\prime} 16^{\prime \prime} \mathrm{S}$ & $47^{\circ} 57^{\prime} 06^{\prime \prime} \mathrm{W}$ \\
\hline IR-05 & Irati & $25^{\circ} 47^{\prime} 12^{\prime \prime} \mathrm{S}$ & $50^{\circ} 25^{\prime} 58^{\prime \prime} \mathrm{W}$ \\
\hline TR-01 & Tremembé & 2 & $23^{\circ} 00^{\prime} 15^{\prime \prime} \mathrm{S}$ & $45^{\circ} 35^{\prime} 05^{\prime \prime} \mathrm{W}$ \\
\hline TR-02 & Tremembé & 4 & $22^{\circ} 57^{\prime} 01^{\prime \prime S}$ & $45^{\circ} 32^{\prime} 26^{\prime \prime} \mathrm{W}$ \\
\hline
\end{tabular}




\subsection{ANÁLISE DO TEOR DE CARBONO ORGÂNICO TOTAL}

Aproximadamente $300 \mathrm{~g}$ de cada amostra foram fragmentadas, secas por $48 \mathrm{~h}$ sob temperatura abaixo de $40{ }^{\circ} \mathrm{C}$ e moídas em moinho de discos. As amostras foram então peneiradas e a fração passante em $0,25 \mathrm{~mm}$ foi utilizada no estudo, de modo a garantir maior homogeneidade granulométrica. Alíquotas de amostra pulverizada com cerca de $10 \mathrm{~g}$ foram separadas para análise do teor de Carbono Orgânico Total (COT). As amostras pulverizadas passaram por ataque com $100 \mathrm{ml}$ de ácido clorídrico a $10 \%$ por $24 \mathrm{~h}$, de modo a eliminar o carbono inorgânico presente. Posteriormente, as amostras foram filtradas com água destilada e secas em estufa a $60{ }^{\circ} \mathrm{C}$ por $48 \mathrm{~h}$. Foram então separados $1,5 \mathrm{~g}$ do material resultante para envio à Central Analítica do Instituto de Química da USP. Utilizou-se equipamento modelo CHN 2400 Perkin-Elmer para a determinação do teor de COT.

\subsection{EXPERIMENTOS DE INCUBAÇÃO}

Para os experimentos de incubação, foram utilizadas alíquotas das amostras de folhelho na fração abaixo de $0,25 \mathrm{~mm}$, obtidas conforme descrito no item 3.2. Os experimentos de incubação foram preparados para se obter curvas de produção de $\mathrm{CH}_{4}$ e $\mathrm{CO}_{2}$ a partir do metabolismo de comunidades microbianas em microcosmos. A geração de ambos os gases foi avaliada ao longo do tempo em experimentos sob diferentes condições. As condições testadas envolveram amostras incubadas a seco, imersas em água, com adição de ácido acético e com renovação do headspace (Figura 3).

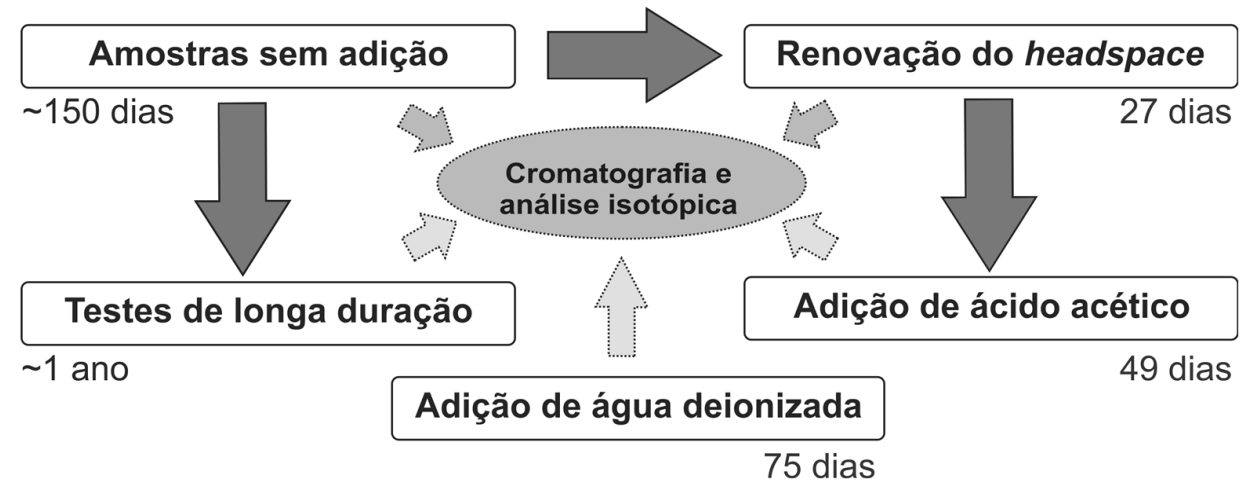

Figura 3 - Etapas dos experimentos de incubação de amostras de folhelhos para produção de $\mathrm{CH}_{4}$ e $\mathrm{CO}_{2}$. 
Triplicatas foram montadas com $15 \mathrm{~g}$ de amostra dispostas em frascos de vidro de $100 \mathrm{ml}$ e seladas com tampas de borracha butílica e selos de alumínio. Os frascos foram então submetidos a séries de evacuação e injeção de $\mathrm{N}_{2}$ de forma a garantir a degaseificação de possíveis contaminantes e simular atmosfera anóxica (Figuras 4 e 5). As amostras foram então armazenadas em ambiente protegido da luz a $25{ }^{\circ} \mathrm{C}$ e sem agitação, para evitar o rompimento de comunidades metanogênicas já formadas (DANNENBERG; WUDLER; CONRAD, 1997).

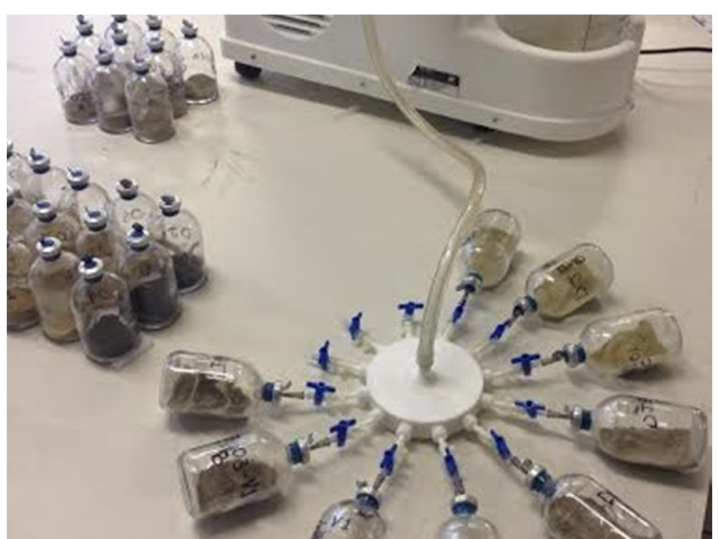

Figura 4 - Etapa do processo de montagem das incubações de folhelho.

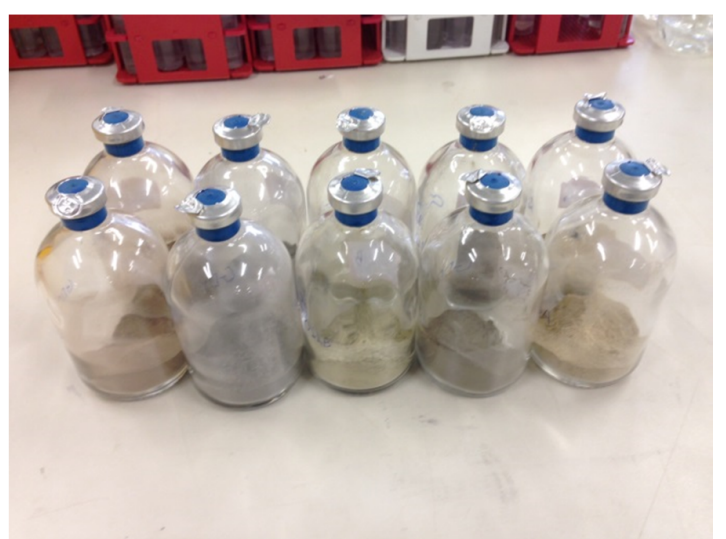

Figura 5 - Amostras acondicionadas em frascos de vidro para testes de incubação.

A amostragem de gases da incubação foi realizada através da injeção de $5 \mathrm{ml}$ de $\mathrm{N}_{2}$ nos frascos e retirada do mesmo volume de gás para análise em cromatógrafo a gás equipado com detector por ionização de chama e com metanizador acoplado (SHIMADZU GC-17A).

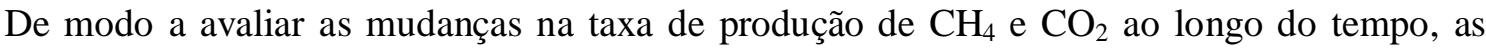
análises foram realizadas entre 1 a 5 vezes por mês, entre Janeiro de 2014 e Julho de 2015. Ao final do experimento, uma das triplicatas foi mantida para testes de longa duração e duas triplicatas foram submetidas à análise em equipamento PICARRO G2201-i Analyzer para avaliar a assinatura isotópica do carbono do metano $\left(\delta^{13} \mathrm{C}_{\mathrm{CH} 4}\right)$ e do dióxido de carbono $\left(\delta^{13} \mathrm{C}_{\mathrm{CO} 2}\right)$ gerados.

As triplicatas submetidas à análise isotópica passaram por nova fase de desgaseificação/reinjeção de $\mathrm{N}_{2}$ para avaliar os efeitos da renovação do headspace nos microcosmos durante período de 27 dias. Após tal etapa, $1 \mathrm{mM}$ de ácido acético dissolvido em água deionizada foi adicionado aos frascos para avaliar possíveis alterações na produção de $\mathrm{CH}_{4}$ e $\mathrm{CO}_{2}$ devido à adição de nutrientes favoráveis à metanogênese. 
Paralelamente, novas incubações com amostras frescas foram montadas em triplicata com a adição de $20 \mathrm{ml}$ de água deionizada com o intuito de verificar a influência da umidade nas comunidades metanogênicas. Novas análises foram realizadas durante período de 75 dias, de acordo com os métodos descritos para os demais experimentos de incubação.

Os dados de produção de $\mathrm{CH}_{4}$ e $\mathrm{CO}_{2}$ foram representados em gráficos de concentração ao longo do tempo e resumidos por estatísticas descritivas. Isto permitiu comparar a produção de $\mathrm{CH}_{4}$ e $\mathrm{CO}_{2}$ sob diferentes condições experimentais. A análise de variância (ANOVA) foi utilizada para comparação do potencial de produção entre as unidades estratigráficas estudadas.

\subsection{ANÁLISE DE ADSORÇÃO DE NITROGÊNIO}

A adsorção de nitrogênio sob baixa pressão é de grande utilidade para avaliação da superfície específica $\left(S_{\text {area }}\right.$ ) e da distribuição de tamanho de poros (DTP) em folhelhos ricos em matéria orgânica (CHEN; XIAO, 2014; LABANI et al., 2013). Tal técnica tem sido intensamente utilizada nos últimos anos, dada a grande importância da estrutura porosa de folhelhos para a caracterização de seu potencial como reservatório não-convencional de hidrocarbonetos (CAO et al., 2015; CHEN; XIAO, 2014; KUILA; PRASAD, 2013; LABANI et al., 2013).

Embora dióxido de carbono e argônio também sejam opções viáveis para a condução de experimentos de adsorção, a utilização de nitrogênio a $77 \mathrm{~K}$, abaixo de seu ponto crítico, é preferível para a avaliação de distribuição da porosidade de materiais em pressões subatmosféricas (GROEN; PEFFER; PÉREZ-RAMÍREZ, 2003). A adoção de métodos de análise em pressões sub-atmosféricas em detrimento de condições supercríticas justifica-se no fato do presente trabalho visar a caracterização da estrutura de poro do meio estudado e não apenas a avaliação de seu potencial de adsorção.

Os resultados obtidos por tal método são eficazes para a determinação da distribuição de tamanho de mesoporos $(2-50 \mathrm{~nm})$. Entretanto, interpretações a respeito da microporosidade $(<2 \mathrm{~nm})$ podem também ser cautelosamente utilizadas (ROUQUEROLT et al., 1994). Avaliações a respeito da macroporosidade (>50 nm), no entanto, são melhores descritas por técnicas complementares como porosimetria por injeção de mercúrio.

A quantificação dos volumes de gás adsorvido e dessorvido em diferentes pressões relativas $\left(\mathrm{P} / \mathrm{P}_{0}\right)$ possibilita a obtenção de isotermas (LABANI et al., 2013). A análise das 
isotermas e da curva de histerese possibilita a interpretação da interação entre meios sólidos e gasosos e pode ser usada para a prognose de algumas características do adsorvente estudado (KUILA, 2013). De acordo com a IUPAC (SING et al., 1985), isotermas de adsorção ou dessorção podem ser categorizadas em seis tipos (I a VI), enquanto os padrões de histerese pode ser classificado em quatro padrões (H1 a H4). A descrição detalhada de tais isotermas de fisiossorção e padrões de histerese revela características importantes do adsorvente e de seus poros (SING et al., 1985).

Com o intuito de se obter informações a respeito da estrutura porosa e $S_{\text {area }}$ das amostras estudadas, frações já pulverizadas e peneiradas $(<0,25 \mathrm{~mm})$ foram submetidas à desgaseificação em equipamento Quantachrome NOVA 2200E por um período de 24 h a 55 ${ }^{\circ} \mathrm{C}$. Após esta etapa, foram realizados experimentos de adsorção e dessorção utilizando nitrogênio a 77,3 K no mesmo equipamento. Todas as recomendações propostas pela IUPAC (SING et al., 1985) foram seguidas durante os procedimentos laboratoriais.

A $S_{\text {area }}$ foi calculada a partir da região linear de Brunauer, Emmet e Teller (BET) para $\mathrm{P} / \mathrm{P}_{0}<0,3$. $\mathrm{O}$ volume total de poro $\left(\mathrm{V}_{\text {poro }}\right)$ foi calculado a partir do ponto mais alto de pressão relativa na isoterma $\left(\mathrm{P} / \mathrm{P}_{0}=0,97\right.$ como padrão). A determinação do volume específico de microporos (VEM), por sua vez, foi estimada pela técnica de $t$-plot utilizando a técnica de espessura de Harkins-Jura (LIPPENS; DE BOER, 1965). Entretanto, deve-se ressaltar que o VEM obtido a partir de tais técnicas deve ser cautelosamente interpretado como dado semiquantitativo, sendo útil principalmente na comparação entre grupos de amostras (KUILA; PRASAD, 2013). Para a comparação entre as unidades estudadas, foi feita análises de variância quando cabível.

Dados relativos à DTP foram obtidos a partir dos métodos da Teoria Funcional de Densidade (DFT) e de Barret, Joyner e Halenda (BJH), ambos utilizados de forma complementar. O método BJH é comumente utilizado para a obtenção da DTP de folhelhos ricos em material orgânico, enquanto o método DFT fornece uma análise mais aprofundada e precisa da DTP na escala de microporosidade (HU et al., 2015; LABANI et al., 2013). A distribuição dos diâmetros de poro obtidos pelo método BJH foi avaliada a partir da isoterma de adsorção, sendo os pontos de $\mathrm{P} / \mathrm{P}_{0}$ abaixo de 0,35 ignorados. Os cálculos para o modelo DFT, por sua vez, basearam-se em núcleo calculado para a adsorção de nitrogênio em poros com formato de fenda em carbono sob temperatura de $77 \mathrm{~K}$. Ressalta-se, porém, que variações composicionais e na estrutura de poro nas amostras estudadas podem não coincidir completamente com o modelo pressuposto pelo núcleo escolhido. 


\section{RESULTADOS}

\subsection{QUANTIFICAÇÃO DE COT E PRODUÇÃO POTENCIAL DE CH $\mathrm{CH}_{4} \mathrm{CO}_{2}$}

Os valores de COT demonstraram grande dispersão, com variação entre 0,18 e 11,11\%. Amostras da Formação Tremembé demonstraram maiores valores para média e desvio padrão ( $\overline{\mathrm{x}}=5,84 \%$; $\sigma=4,66 \%)$, seguidas respectivamente pelas amostras da Formação Irati $(\overline{\mathrm{x}}=3,87 \% ; \sigma=2,00 \%)$ e Formação Ponta Grossa $(\overline{\mathrm{x}}=1,18 \% ; \sigma=0,82 \%)$.

As médias das taxas de produção de $\mathrm{CH}_{4}$ e $\mathrm{CO}_{2}$ das unidades estudadas variam em termos das condições de incubação. Testes a seco apresentaram resultados heterogêneos, com produção de $\mathrm{CH}_{4}$ entre $2,50 \mathrm{ml} / \mathrm{t}$ e $111,31 \mathrm{ml} / \mathrm{t}$ durante período normalizado de 150 dias (Figura 6). A produção total de $\mathrm{CH}_{4}$ por unidade geológica estudada para os testes a seco demonstrou valores médios de $12,3 \mathrm{ml} / \mathrm{t}$ para a Formação Ponta Grossa, 52,7 ml/t para a Formação Irati e 20,8 ml/t para a Formação Tremembé. A produção variou entre 5,7 ml/t e $111,3 \mathrm{ml} / \mathrm{t}$ para a Formação Irati, 2,5 ml/t e 54,4 ml/t para a Formação Ponta Grossa e 2,8 ml/t e 33,1 ml/t para a Formação Tremembé. As grandes variações obtidas entre amostras da mesma unidade estratigráfica indicam heterogeneidade dos controles sobre o potencial de produção de $\mathrm{CH}_{4}$ e $\mathrm{CO}_{2}$.

As taxas de geração de $\mathrm{CH}_{4}$ a seco demonstraram declínio acentuado ao longo do tempo, com média de $0,44 \mathrm{ml} / \mathrm{t}$.d nos primeiros 30 dias para $0,16 \mathrm{ml} / \mathrm{t} . \mathrm{d}$ ao considerar-se todo o período normalizado de 150 dias.

A produção de $\mathrm{CO}_{2}$ ao longo dos testes a seco é detalhada na Figura 6. As amostras da Formação Ponta Grossa produziram $23,8 \times 10^{3} \mathrm{ml} / \mathrm{t}$ de $\mathrm{CO}_{2}$ ao longo do teste, enquanto que

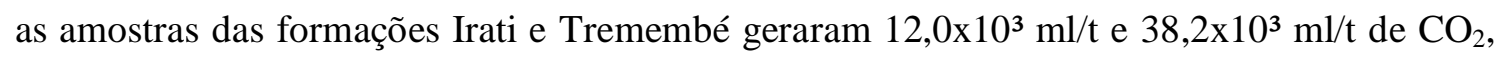
respectivamente.

Após a renovação do headspace por evacuação e reinjeção de nitrogênio, notou-se mudança no padrão de produção de $\mathrm{CH}_{4}$ e $\mathrm{CO}_{2}$. Apenas amostras das formações Irati (IR-01 e IR-02) e Tremembé (TR-02-4) demonstraram concentração de $\mathrm{CH}_{4}$ acima dos limites de detecção, com produção média acumulada de 7,8 ml/t ao longo dos 30 dias normalizados de análise. A produção de $\mathrm{CO}_{2}$ foi detectada em 5 de 9 incubações analisadas, sendo que as amostras TR-02-2 e TR-02-4 da Formação Tremembé apresentaram as maiores taxas de produção, $2,9 \times 10^{3} \mathrm{ml} / \mathrm{t} . \mathrm{d}$ e $1,1 \times 10^{3} \mathrm{ml} / \mathrm{t} . \mathrm{d}$, respectivamente. 


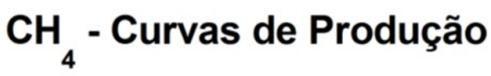

$\mathrm{CO}_{2}$ - Curvas de Produção
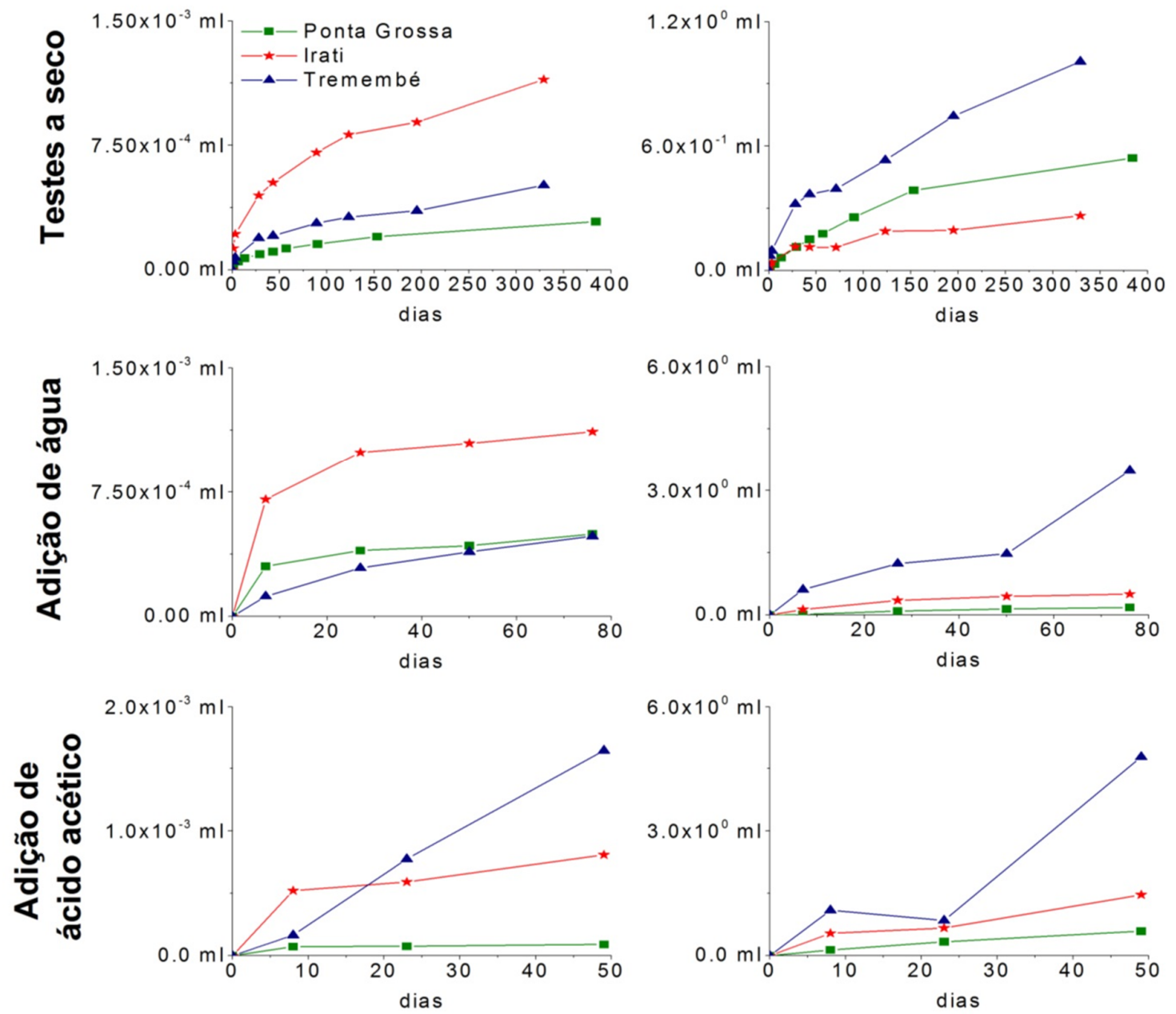

Figura 6 - Curvas de produção de $\mathrm{CH}_{4}$ e $\mathrm{CO}_{2}$ obtidas a partir de incubações a seco, com adição de água e com adição de ácido acético.

Testes de longa duração foram normalizados para o período de 1 ano, de modo a facilitar a comparação entre as 3 diferentes unidades estudadas. Amostras da Formação Irati demonstraram a maior produção média de $\mathrm{CH}_{4}(79,5 \mathrm{ml} / \mathrm{t})$, sendo que a amostra com maior produção atingiu $152 \mathrm{ml} / \mathrm{t}$ (IR-02). A Formação Ponta Grossa apresentou produção anual média de 17,7 ml/t, enquanto os folhelhos da Formação Tremembé atingiram a média anual de $35,4 \mathrm{ml} / \mathrm{t}$.

Diferente do $\mathrm{CH}_{4}$, as maiores taxas de produção de $\mathrm{CO}_{2}$ nos experimentos de incubação de longa duração foram detectadas para amostras da Formação Tremembé. As produções de $\mathrm{CO}_{2}$ para as formações Ponta Grossa, Irati e Tremembé foram de 90,6 ml/t.d, 
$50,3 \mathrm{ml} / \mathrm{t}$.d e 191,5 ml/t.d, respectivamente. As taxas de geração de $\mathrm{CH}_{4} \mathrm{e} \mathrm{CO}_{2}$ mantiveram o padrão de queda ao longo do tempo. Ao final do período de 1 ano, as taxas de geração foram aproximadamente $35 \%$ menores que os valores obtidos nos primeiros 150 dias de incubação.

As concentrações de $\mathrm{CH}_{4}$ e $\mathrm{CO}_{2}$ foram avaliadas nas incubações após a adição de ácido acético nos dias 0, 8, 23 e 49 (Figura 6). Na presença de ácido ácetico, a produção total ao longo de 49 dias variou entre 2,93 ml/t (Formação Ponta Grossa) e 253,3 ml/t (Formação Tremembé). Amostras da Formação Tremembé demonstraram a maior produção de $\mathrm{CH}_{4}$, com média de 104,9 ml/t (2,14 ml/t.d como taxa de produção média) ao final dos 49 dias. As formações Irati e Ponta Grossa produziram, em média, 51,6 ml/t e 5,8 ml/t de $\mathrm{CH}_{4}$. Destaca-se a alta produção de $\mathrm{CO}_{2}$ na presença de ácido acético, com taxas médias de $0,77 \times 10^{3} \mathrm{ml} / \mathrm{t} . \mathrm{d}$

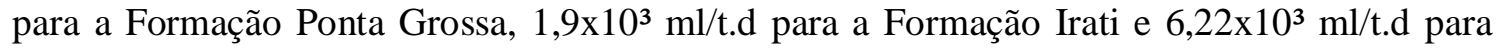
amostras da Formação Tremembé. A adição de ácido acético dissolvido em água aparenta também ser suficiente para reiniciar e aumentar a produção de $\mathrm{CH}_{4}$ nos microcosmos avaliados.

Incubações montadas com a adição de $20 \mathrm{ml}$ de água demonstraram produção de $\mathrm{CH}_{4}$ de até 179,2 ml/t. As produções médias para as formações Ponta Grossa, Tremembé e Irati foram 30,6 ml/t, 68,8 ml/t e 29,8 ml/t, respectivamente, em um período normalizado de 75 dias. Taxas médias de produção de metano em condição aquosa foram 54\% maior que experimentos a seco durante os 30 primeiros dias de incubação.

Conforme obtido em outros cenários, a taxa de geração de metano foi reduzida ao longo do tempo para incubações com adição de água, com médias de 1,18 m1/t.d, após 30 dias de incubação, e 0,57 ml/t.d, após 75 dias de incubação. Amostras da Formação Tremembé apresentaram a maior produção de $\mathrm{CO}_{2}$ entre as unidades estudadas, com taxas médias de 2,9x $10^{3} \mathrm{ml} / \mathrm{t} . \mathrm{d}$ durante os 75 dias de experimento. As formações Irati e Ponta Grossa produziram quantias significativamente menores neste experimento, com taxas de produção respectivamente $86 \%\left(0,4 \times 10^{3} \mathrm{ml} / \mathrm{t} . \mathrm{d}\right)$ e $95 \%\left(0,15 \times 10^{3} \mathrm{ml} / \mathrm{t} . \mathrm{d}\right)$ abaixo do produzido pelas amostras da Formação Tremembé. Testes com adição de água também demonstraram maior produção de $\mathrm{CO}_{2}$ em comparação com testes a seco, com aumento médio na produção equivalente a $151 \%$, durante os primeiros 30 dias de incubação.

$\mathrm{O}$ sumário das taxas de produção de $\mathrm{CH}_{4} \mathrm{e} \mathrm{CO}_{2}$ ao longo dos primeiros 30 dias de incubação para testes a seco, com renovação do headspace, adição de ácido acético e com adição de água é apresentado na Tabela 2. 
Tabela 2 - Valores médios (̄), mínimos (mín), máximos (máx) e desvio padrão $(\sigma)$ do teor de COT e da taxa de produção (TP) de $\mathrm{CH}_{4}$ e $\mathrm{CO}_{2}$ sob condições a seco (SC), após renovação do headspace (R), após adição de ácido acético (AA) e com adição de água (CA).

\begin{tabular}{|c|c|c|c|c|c|c|c|c|c|c|}
\hline \multirow{3}{*}{\multicolumn{2}{|c|}{ Unidade }} & \multirow{4}{*}{$\begin{array}{c}\text { COT } \\
\% \\
1,18\end{array}$} & \multicolumn{2}{|c|}{ SC TP } & \multicolumn{2}{|c|}{ R TP } & \multicolumn{2}{|c|}{ AA $\mathbf{T P}$} & \multicolumn{2}{|c|}{ CA TP } \\
\hline & & & \multirow{2}{*}{$\mathrm{CH}_{4}$} & \multirow[t]{2}{*}{$\mathrm{CO}_{2}$} & \multirow{2}{*}{$\mathrm{CH}_{4}$} & \multirow{2}{*}{$\frac{\mathrm{CO}_{2}}{\mathrm{ml}}$} & \multirow{2}{*}{$\frac{\mathrm{CH}_{4}}{\text { t.d }}$} & \multirow[t]{2}{*}{$\mathrm{CO}_{2}$} & \multirow[t]{2}{*}{$\mathrm{CH}_{4}$} & \multirow[t]{2}{*}{$\mathrm{CO}_{2}$} \\
\hline & & & & & & & & & & \\
\hline \multirow{4}{*}{$\begin{array}{l}\text { Ponta } \\
\text { Grossa }\end{array}$} & $\overline{\mathbf{x}}$ & & 0,20 & 244,00 & $\mathbf{0 , 0 0}$ & 19,53 & 0,17 & 840,65 & 0,84 & 211,07 \\
\hline & $\sigma$ & 0,82 & 0,33 & 544,94 & 0,00 & 27,62 & 0,12 & 352,57 & 0,67 & 126,91 \\
\hline & mín & 0,22 & 0,01 & 16,93 & 0,00 & 0,00 & 0,03 & 437,86 & 0,12 & 105,61 \\
\hline & máx & 3,11 & 1,23 & 2021,43 & 0,00 & 58,59 & 0,32 & 1296,56 & 1,74 & 389,57 \\
\hline \multirow{4}{*}{ Irati } & $\overline{\mathbf{x}}$ & 3,87 & 0,96 & 256,19 & 0,19 & 38,56 & 1,35 & 1836,60 & 2,08 & $\mathbf{7 5 8 , 9 7}$ \\
\hline & $\boldsymbol{\sigma}$ & 2,00 & 1,04 & 209,46 & 0,14 & 54,53 & 1,27 & 683,53 & 2,44 & 179,64 \\
\hline & mín & 1,09 & 0,12 & 73,11 & 0,00 & 0,00 & 0,39 & 1234,19 & 0,22 & 510,60 \\
\hline & máx & 6,34 & 3,17 & 712,46 & 0,35 & 115,68 & 3,15 & 2792,51 & 5,53 & 929,43 \\
\hline \multirow{4}{*}{ Tremembé } & $\overline{\mathbf{x}}$ & 5,84 & $\mathbf{0 , 4 0}$ & 716,27 & $\mathbf{0 , 0 7}$ & 1418,82 & 2,10 & 3974,15 & 0,64 & 2644,80 \\
\hline & $\sigma$ & 4,66 & 0,25 & 792,25 & 0,10 & 1087,88 & 1,89 & 2701,03 & 0,41 & 1606,74 \\
\hline & mín & 0,18 & 0,07 & 146,58 & 0,00 & 293,98 & 0,05 & 854,57 & 0,09 & 516,52 \\
\hline & máx & 11,11 & 0,72 & 2450,80 & 0,21 & 2890,24 & 4,61 & 7442,99 & 1,05 & 4398,32 \\
\hline
\end{tabular}

\subsection{CARACTERÍSTICAS ISOTÓPICAS DO CH $\mathrm{CH} \mathrm{CO}_{2}$}

Assim como obtido nos resultados de produção biogênica de $\mathrm{CH}_{4} \mathrm{e} \mathrm{CO}_{2}$, análises de $\delta^{13} \mathrm{C}$ também apresentaram valores com grande dispersão. $\mathrm{O} \delta^{13} \mathrm{C}_{\mathrm{CH} 4}$ para os testes realizados a seco variou entre -109 e $-22 \%$ o (-64\%o em média) e o $\delta^{13} \mathrm{C}_{\mathrm{CO} 2}$ entre $-30 \%$ e $0 \%$ o $(-18 \%$ om média). Diferenças significativas foram notadas entre as formações estudadas, conforme destacado pela Figura 7.

Nos testes de longa duração, o $\delta^{13} \mathrm{C}_{\mathrm{CH} 4}$ variou entre -180 e -24\%o (-66\%o em média), enquanto o $\delta^{13} \mathrm{C}_{\mathrm{CO} 2}$ variou entre $-30 \%$ e $4 \%$, com média de $-17 \%$. Entre as unidades estudadas, as médias obtidas para $\delta^{13} \mathrm{C}_{\mathrm{CH} 4}$ and $\delta^{13} \mathrm{C}_{\mathrm{CO} 2}$ foram, respectivamente, $-85 \%$ e $-24 \%$ o para a Formação Ponta Grossa, -38\%o e -8\%o para a Formação Irati e -91\%o e -12\%o para a Formação Tremembé. Conforme demonstrado na Figura 7, diferenças pouco significativas foram reconhecidas na comparação das médias entre os resultados obtidos nos testes a seco e de longa duração.

Valores de $\delta^{13} \mathrm{C}_{\mathrm{CH} 4}$ foram obtidos para 3 amostras incubadas sob presença de ácido acético, os quais variaram de $-51 \%$ (TR-02-2) a $-43 \%$ (IR-01). As análises para $\mathrm{CO}_{2}$, 
entretanto, demonstraram $\delta^{13} \mathrm{C}_{\mathrm{CO} 2}$ entre $-72 \%$ e $-15 \%$, com média de $-34 \%$. Assinaturas isotópicas do $\mathrm{CO}_{2}$ demonstraram médias de $-35 \%$ o para a Formação Ponta Grossa, -38\%o para a Formação Irati e -16\%o para a Formação Tremembé, diferenças similares às observadas em outros experimentos.

As assinaturas isotópicas obtidas para os testes com adição de água também apresentaram alta dispersão e seguiram o padrão dos outros testes. Os valores de $\delta^{13} \mathrm{C}_{\mathrm{CH} 4}$ variaram entre $-142 \%$ e $-35 \%$ (média de $-82 \%$ ). Valores de $\delta^{13} \mathrm{C}_{\mathrm{CO} 2}$ variaram entre $-27 \%$ o e 13\%o (média de -19\%o). Para este caso, as amostras da Formação Tremembé demonstraram significativa diferença em relação às características de outras unidades, com valor médio de $140 \%$ para $\delta^{13} \mathrm{C}_{\mathrm{CH} 4}$ e $-13 \%$ para $\delta^{13} \mathrm{C}_{\mathrm{CO} 2}$. As formações Ponta Grossa e Irati, por outro lado, apresentaram média de $-48 \%$ e $-58 \%$ o para $\delta^{13} \mathrm{C}_{\mathrm{CH} 4}$ e $-23 \%$ e $-17 \%$ o para $\delta^{13} \mathrm{C}_{\mathrm{CO} 2}$, respectivamente.
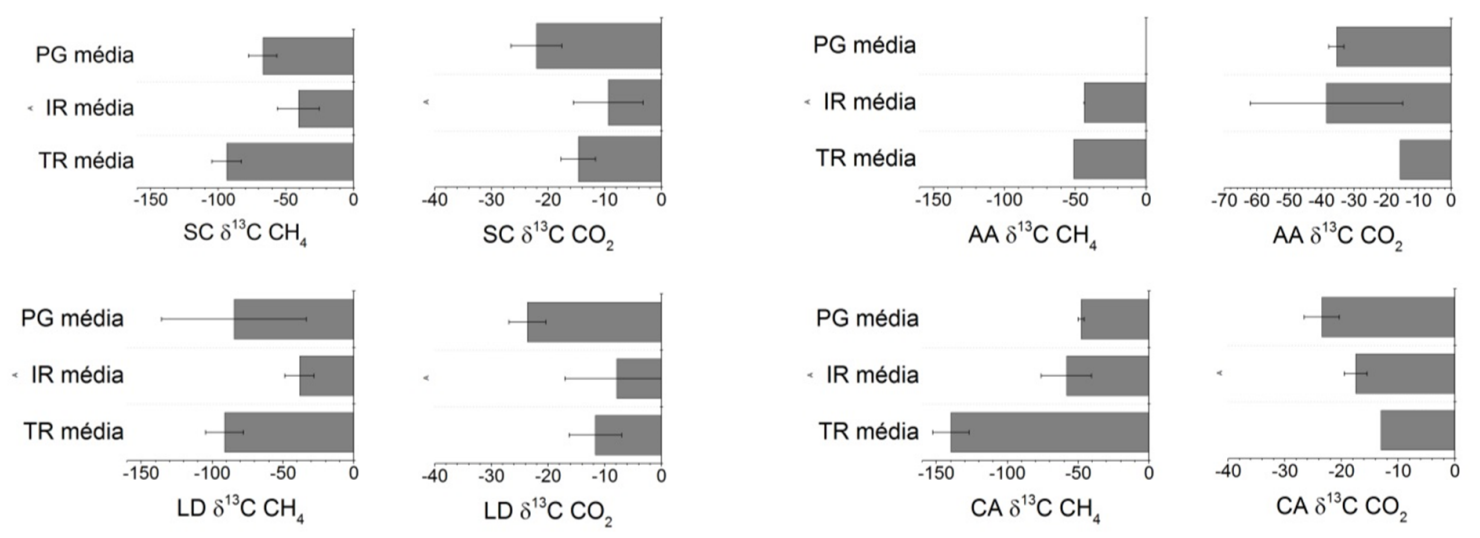

Figura 7 - Média e desvio padrão dos valores de $\delta^{13} \mathrm{C}$ para $\mathrm{CH}_{4}$ e $\mathrm{CO}_{2}$ produzidos em testes a seco (SC), de longa duração (LD), com adição de ácido acético (AA) e com adição de água

(CA).

\subsection{ANÁLISE DE ADSORÇÃO DE NITROGÊNIO}

\subsubsection{Isotermas de adsorção e dessorção}

As isotermas de adsorção/dessorção de amostras selecionadas das formações Ponta Grossa, Irati e Tremembé são apresentadas na Figura 8. 

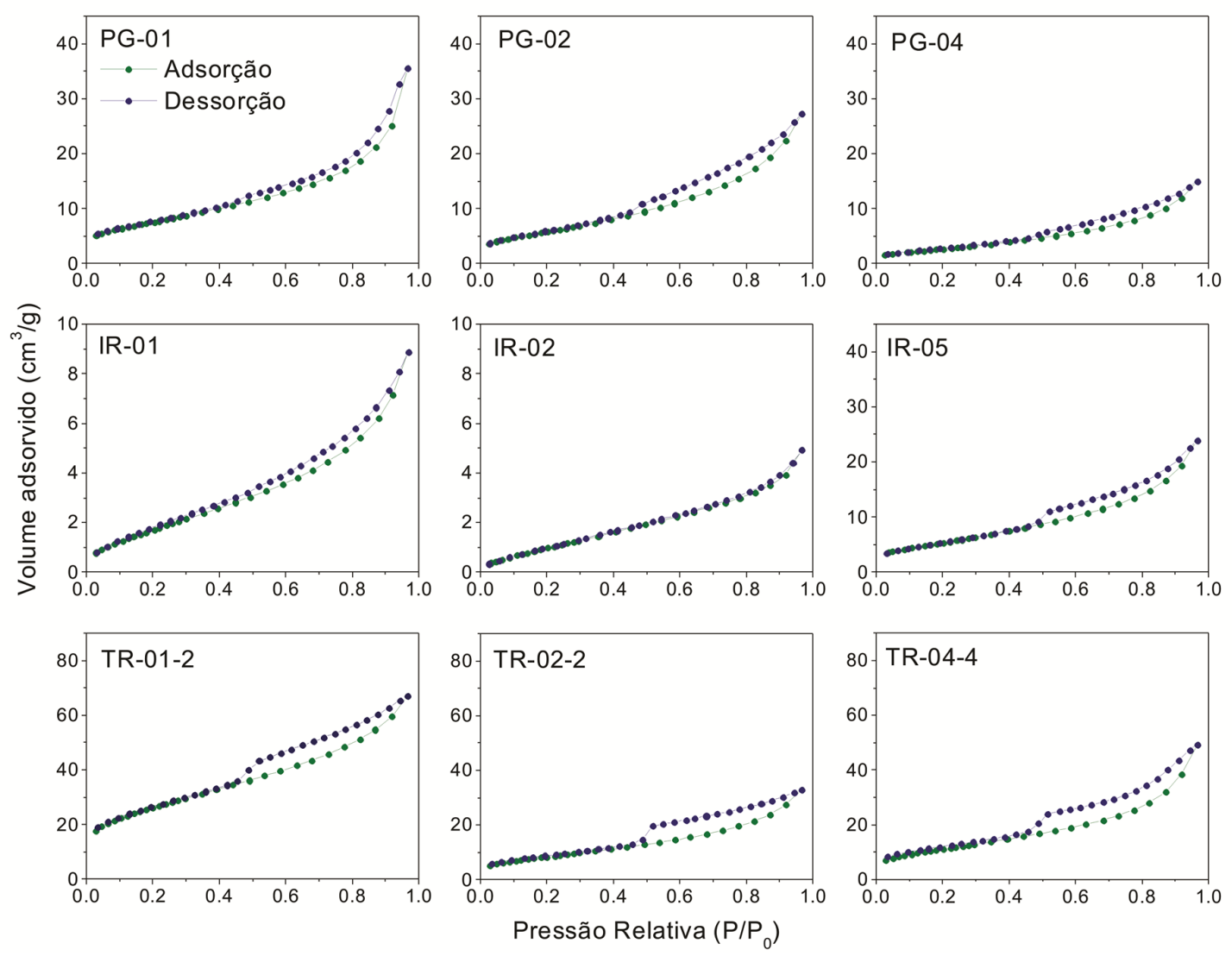

Figura 8 - Isotermas obtidas a partir dos ensaios de adsorção de nitrogênio para amostras das formações Ponta Grossa (PG), Irati (IR) e Tremembé (TR). Três amostras de cada unidade foram selecionadas com o intuito de exemplificar os padrões obtidos.

De acordo com recomendações da International Union of Pure and Applied Chemistry (IUPAC), os resultados obtidos pelo presente estudo podem ser classificados como isotermas do tipo II e IV, com curva de histerese e grande captação em maiores $\mathrm{P} / \mathrm{P}_{0}$ (SING et al., 1985). Apesar de trabalhos recentes escolherem apenas um dos modelos de isotermas recomendados pela IUPAC, ROUQUEROL et al. (1999) sugere classificação (Tipo Ilb) para o padrão de isoterma obtido no presente estudo. Isotermas Tipo IIb são geralmente associadas à argilitos e lamitos em que predominam poros com formato de fenda. Estas rochas apresentam a ramificação de adsorção com formato similar ao Tipo II clássico associado à histerese do tipo H3. Nota-se também a ausência de platô característico em áreas de maior $\mathrm{P} / \mathrm{P}_{0}$ como observado em isotermas do Tipo IV (ROUQUEROL; ROUQUEROL; SING, 1999). A ausência do platô indica volume significativo de macroporos, enquanto a histerese relaciona-se à presença de mesoporos (SING et al., 1985). As isotermas obtidas neste trabalho indicam que amostras da Formação Tremembé possuem fechamento de histerese mais 
proeminente, sugerindo elevado volume de poros com diâmetro abaixo de $4 \mathrm{~nm}$ (GREGG; SING., 1982). Amostras da Formação Irati (IR-04, IR-03-1 e IR-03-2) demonstram histerese de baixa pressão, geralmente associada a estrangulamento de poros ou incorporações de moléculas do adsorvato (SING et al., 1985).

\subsubsection{Superfície específica e volume de poro}

Valores obtidos para $S_{\text {area }}$ baseados em BET multipontos variam entre $5 \mathrm{~m}^{2} / \mathrm{g}$ e 109 $\mathrm{m}^{2} / \mathrm{g}$ (Tabela 3). As formações Ponta Grossa, Irati e Tremembé demonstraram diferenças significativas (ANOVA, $F(2,21)=15,95 ; \mathrm{p}<0,01$ ), com valores de $S_{\text {area }}$ de $23 \mathrm{~m}^{2} / \mathrm{g}, 9 \mathrm{~m}^{2} / \mathrm{g}$ e 61 $\mathrm{m}^{2} / \mathrm{g}$, respectivamente (Figura 9).

Considerando todas as amostras coletadas, valores de $\mathrm{V}_{\text {poro }}$ variaram entre 0,76

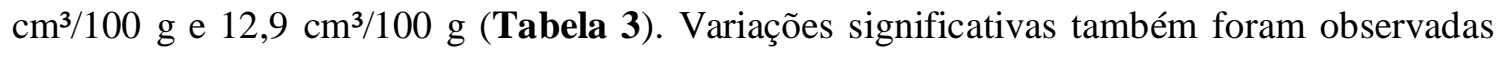
para $\mathrm{V}_{\text {poro }}($ ANOVA, $\mathrm{F}(2,21)=21,62 ; \mathrm{p}<0,01)$, sendo que a Formação Irati demonstrou os menores volumes entre as unidades estudadas, com 1,6 $\mathrm{cm}^{3} / 100 \mathrm{~g}$ de média e sem VEM detectável. A Formação Ponta Grossa possui média de $4,4 \mathrm{~cm}^{3} / 100 \mathrm{~g}$ de $\mathrm{V}_{\text {poro, }}$, também sem VEM detectável. A Formação Tremembé, por sua vez, demonstrou média de $8,3 \mathrm{~cm}^{3} / 100 \mathrm{~g}$ de $\mathrm{V}_{\text {poro }}$ (Figura 9), com 0,4 $\mathrm{cm}^{3} / 100 \mathrm{~g}$ de VEM (aproximadamente $5 \%$ do $\mathrm{V}_{\text {poro }}$ ) detectado por técnica t-plot. Observou-se correlação negativa entre as taxas de produção de $\mathrm{CH}_{4}$ e o volume de poro obtido para as amostras avaliadas (Figura 10). 
Tabela 3 - Variáveis da estrutura de poros de amostras de folhelho das unidades estudadas.

Além dos valores de $S_{\text {area }}, V_{\text {poro }}$ e VEM, são também apresentados o diâmetro médio de poro $\left(\mathrm{D}_{\text {poro }}\right)$, o volume de poro calculado a partir do método DFT ( $\left.\mathrm{V}_{\text {total }} \mathrm{DFT}\right)$ e a razão de VEM por $\mathrm{V}_{\text {total }}$ DFT (VEM razão DFT).

\begin{tabular}{|c|c|c|c|c|c|c|c|}
\hline Amostra & $\begin{array}{c}\mathbf{S}_{\text {area }} \\
\text { BET }\left(\mathrm{m}^{2} / \mathrm{g}\right)\end{array}$ & $\begin{array}{c}\text { D }_{\text {poro }} \\
\text { BJH }(\mathbf{n m})\end{array}$ & $\begin{array}{c}\text { D poro } \\
\text { DFT (nm) }\end{array}$ & $\begin{array}{c}\mathbf{V}_{\text {poro }} \\
\mathrm{P} / \mathrm{P}_{0}=0,97 \\
\left(\mathrm{~cm}^{3} / 100 \mathrm{~g}\right)\end{array}$ & $\begin{array}{l}\text { VEM DFT } \\
\left(\mathrm{cm}^{3} / 100 \mathrm{~g}\right)\end{array}$ & $\begin{array}{c}\mathbf{V}_{\text {total }} \\
\text { DFT } \\
\left(\mathrm{cm}^{3} / 100 \mathrm{~g}\right)\end{array}$ & \begin{tabular}{|c|} 
VEM $_{\text {razão }}$ \\
DFT \\
$(\%)$ \\
\end{tabular} \\
\hline PG-01 & 27 & 3,35 & 3,63 & 5,49 & 0,44 & 4,8 & 9,2 \\
\hline PG-02 & 22 & 3,35 & 3,79 & 4,21 & 0,18 & 3,8 & 4,7 \\
\hline PG-03-1 & 21 & 3,37 & 3,79 & 4,04 & 0,19 & 3,6 & 5,4 \\
\hline PG-03-2 & 21 & 3,35 & 3,79 & 3,33 & 0,27 & 3 & 8,8 \\
\hline PG-03-3 & 23 & 3,36 & 3,79 & 4,17 & 0,31 & 3,7 & 8,4 \\
\hline PG-03-4 & 26 & 3,36 & 3,79 & 3,96 & 0,38 & 3,5 & 10,8 \\
\hline PG-04 & 10 & 3,4 & 4,15 & 2,32 & 0,03 & 2,1 & 1,6 \\
\hline PG-05 & 33 & 3,38 & 3,79 & 5,81 & 0,52 & 5,2 & 10,0 \\
\hline PG-06-1 & 24 & 3,35 & 3,63 & 4,12 & 0,39 & 3,7 & 10,6 \\
\hline PG-06-2 & 17 & 3,04 & 3,79 & 3,02 & 0,19 & 2,7 & 6,9 \\
\hline PG-06-3 & 27 & 3,36 & 3,63 & 6,59 & 0,24 & 5,7 & 4,3 \\
\hline PG-06-4 & 24 & 3,37 & 4,75 & 6,00 & 0,19 & 5,4 & 3,5 \\
\hline IR-01 & 7 & 3,04 & 2,77 & 1,37 & 0,03 & 1,2 & 2,2 \\
\hline IR-02 & 5 & 3,09 & 2,77 & 0,76 & 0,00 & 0,6 & 0,0 \\
\hline IR-03-1 & 6 & 3,05 & 4,15 & 1,15 & 0,06 & 1 & 6,4 \\
\hline IR-03-2 & 7 & 3,05 & 2,77 & 0,96 & 0,13 & 0,8 & 15,7 \\
\hline IR-04 & 8 & 3,38 & 4,15 & 1,41 & 0,09 & 1,2 & 7,7 \\
\hline IR-05 & 20 & 3,4 & 4,15 & 3,69 & 0,19 & 3,3 & 5,7 \\
\hline TR-01-1 & 109 & 3,35 & 4,15 & 12,9 & 2,24 & 11,9 & 18,8 \\
\hline TR-01-2 & 93 & 3,34 & 4,15 & 10,3 & 2,13 & 9,5 & 22,4 \\
\hline TR-02-1 & 61 & 3,34 & 4,34 & 8,15 & 1,21 & 7,5 & 16,1 \\
\hline TR-02-2 & 31 & 3,34 & 4,15 & 5,09 & 0,35 & 4,7 & 7,5 \\
\hline TR-02-3 & 32 & 3,34 & 4,15 & 5,69 & 0,31 & 5,2 & 6,0 \\
\hline TR-02-4 & 41 & 3,36 & 3,97 & 7,64 & 0,58 & 7 & 8,3 \\
\hline
\end{tabular}


A

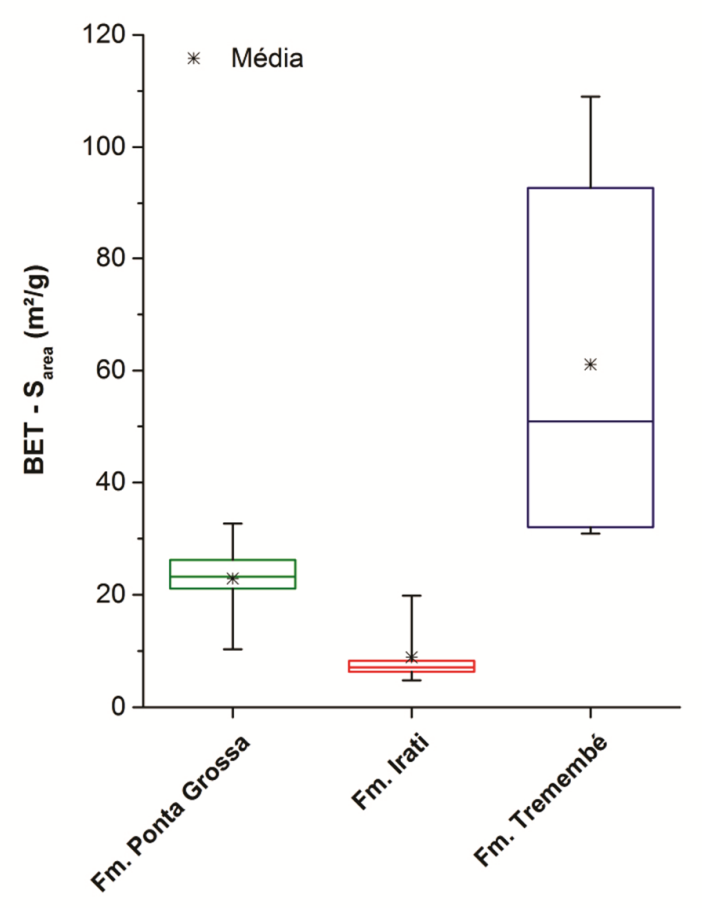

B

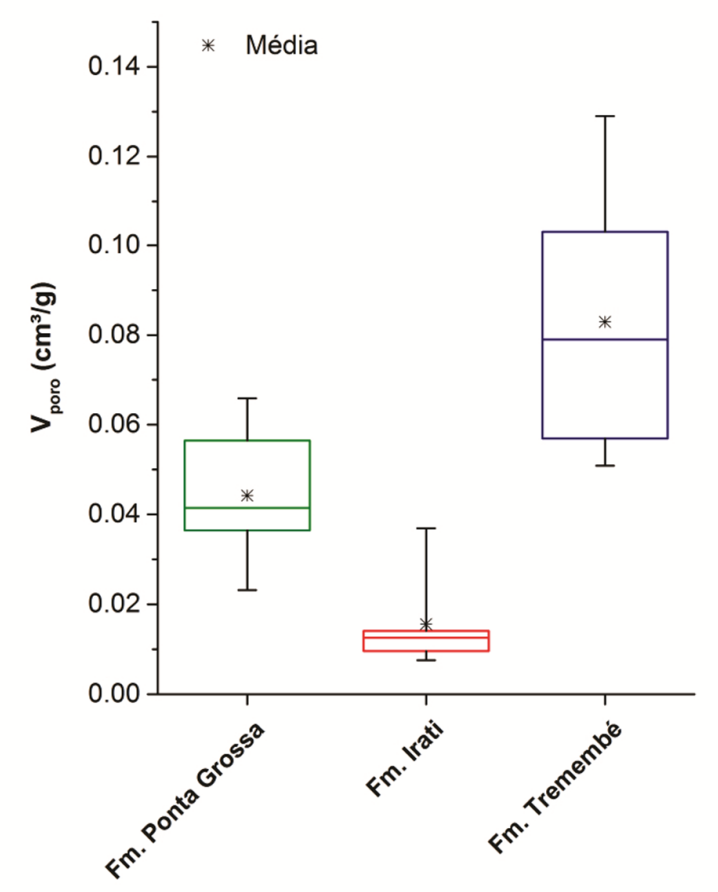

Figura 9 - Gráficos boxplot com resultados de $S_{\text {area }}(A, n=24)$ e $V_{\text {poro }}(B, n=24)$ calculados no ponto de maior $\mathrm{P} / \mathrm{P}_{0}$.

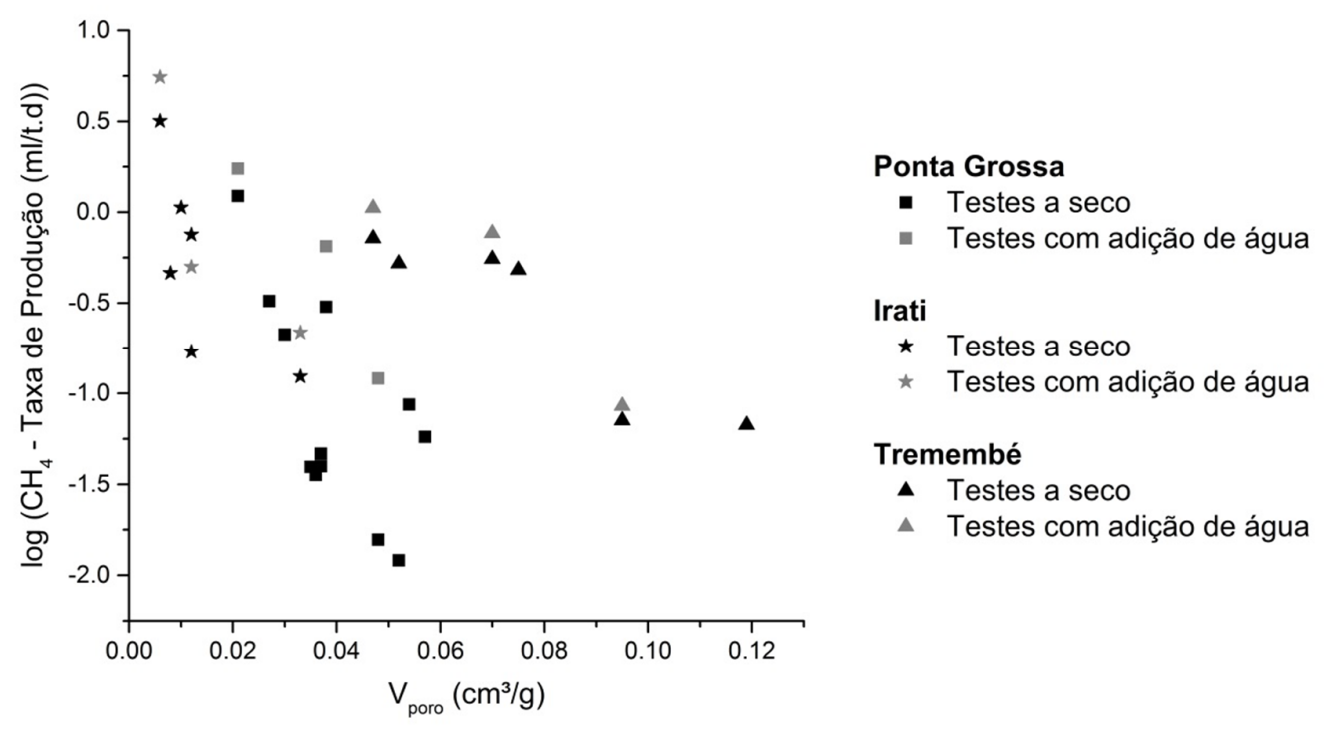

Figura 10 - Relação entre produção de $\mathrm{CH}_{4}$ e $\mathrm{V}_{\text {poro }}(\mathrm{r}=-0,42 ; \mathrm{p}=0,01)$. Dados de produção utilizados a partir de testes a seco e com adição de água. 


\subsubsection{Distribuição do tamanho de poro: Métodos BJH e DFT}

No presente trabalho, a DTP de amostras de folhelhos ricos em materia orgânica foi investigada por dois métodos complementares, BJH e DFT, conforme demonstrado pela Tabela 3.

As médias dos diâmetros de poro obtidos indicam diferença significativa entre as unidades estudadas para os métodos BJH (ANOVA, F(2,21)=5,59, p<0,05) e DFT (ANOVA, $\mathrm{F}(2,21)=3,82 ; \mathrm{p}<0,05)$. Para o método $\mathrm{BJH}$, os valores médios por unidade foram 3,34 nm $(\sigma=0,09 \mathrm{~nm})$ para a Formação Ponta Grossa, $3,17 \mathrm{~nm}(\sigma=0,17 \mathrm{~nm})$ para a Formação Irati e $3,35 \mathrm{~nm}(\sigma=0,01 \mathrm{~nm})$ para a Formação Tremembé. O método DFT, por outro lado, apresenta diâmetro de poro médio de 3,86 $\mathrm{nm}(\sigma=0,09 \mathrm{~nm})$ para a Formação Ponta Grossa, 3,46 nm $(\sigma=0,17 \mathrm{~nm})$ para a Formação Irati e $4,15 \mathrm{~nm}(\sigma=0,01 \mathrm{~nm})$ para amostras da Formação Tremembé. Cabe ressaltar que o diâmetro médio de poro obtido para ambas as análises são significativamente menores do que resultados de trabalhos anteriores para outras unidades (CAO et al., 2015; LABANI et al., 2013; LIU; XIONG; LIANG, 2015; PAN et al., 2015; YANG; NING; LIU, 2014)

A representação gráfica do DTP obtida a partir da ramificação de adsorção pelo método BJH demonstra grande dispersão entre 2 e $40 \mathrm{~nm}$, sem indicar padrão detectável entre distribuições unimodais, bimodais ou multi-modais (Figura 11). DTPs obtidos pelo método DFT, entretanto, destacam diferenças entre dois grupos de amostras. O primeiro grupo, composto pelas formações Irati e Ponta Grossa, apresenta distribuição mais ampla e menor volume de poro. As amostras da Formação Tremembé compõem o segundo grupo e destacam-se por apresentar picos concentrados em bandas menores que $4 \mathrm{~nm}$. Ressalta-se, entretanto, que resultados quantitativos relativos ao volume de microporos devem ser considerados com cautela, dada a limitação da difusão das moléculas de nitrogênio em microporos de adsorventes compostos por carbono (JAGIELLO; THOMMES, 2004; LAY; YU-YOU; TATSUYA, 1997). 

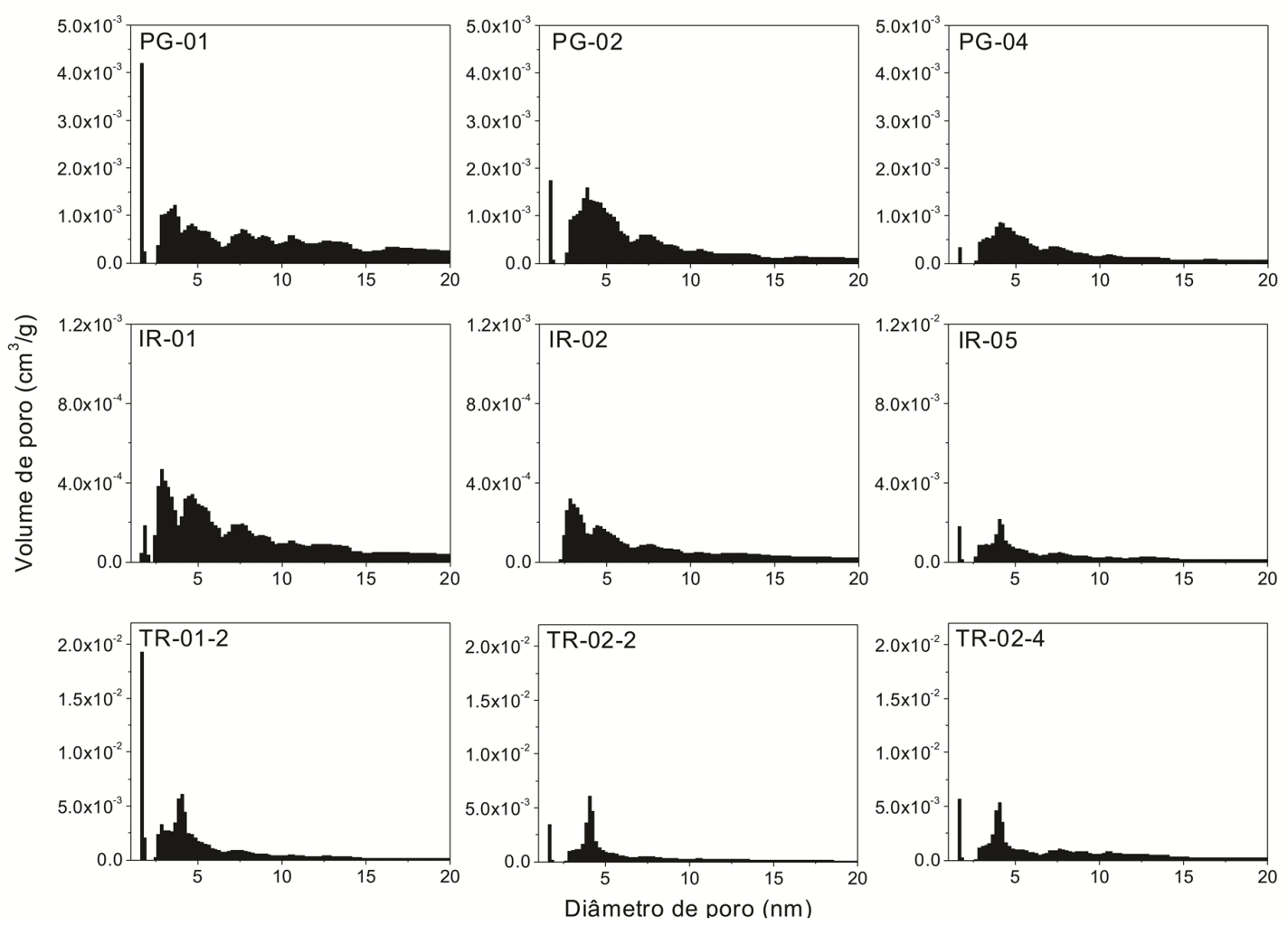

Figura 11 - Histogramas de PSD de nove amostras representativas dos padrões obtidos para cada unidade estudada. A avaliação do diâmetro de poros a partir de análises DFT demonstra maior amplitude de distribuição para as amostras das formações Ponta Grossa e Irati. 


\section{DISCUSSÃO}

\subsection{FATORES CONTROLADORES DA GERAÇÃO DE $\mathrm{CH}_{4} \mathrm{E} \mathrm{CO}_{2}$}

A geração de metano biogênico em ambientes com baixa concentração de sulfato geralmente mantém fase inicial de crescimento exponencial seguida de fase de platô, com zona de inflexão suave possivelmente associada a mudanças no consumo de substrato ou na via metabólica (WHITICAR, 1999). Na ausência de competição com bactérias redutoras de sulfato, a metanogênese por redução de $\mathrm{CO}_{2}$ é menos significativa em comparação com a via acetotrófica. Com o esgotamento de acetato, o metabolismo de $\mathrm{CO}_{2}$ adquire maior importância e controla as taxas de produção de metano (WHITICAR, 1999).

Conforme demonstrado na Figura 6, as incubações preparadas com amostras das formações Irati e Ponta Grossa exibem queda na taxa de produção ao longo do tempo. Incubações elaboradas a partir de amostras da Formação Tremembé, entretanto, apesar de breves picos de produção, apresentam tendência mais próxima de linear na geração de metano e decaimento abrupto de geração nas curvas de produção de $\mathrm{CO}_{2}$.

Alterações no metabolismo microbial dentro dos microcosmos teriam como consequência a alteração nos valores de $\delta^{13} \mathrm{C}_{\mathrm{CH} 4}$ and $\delta^{13} \mathrm{C}_{\mathrm{CO} 2}$, uma vez que as duas vias metabólicas (acetato e $\mathrm{CO}_{2}$ ) possuem diferentes fatores de fracionamento isotópico (WHITICAR, 1999). No presente trabalho, comparações entre testes a seco de médio e longo prazo demonstraram variações pouco significativas nos valores finais de $\delta^{13} \mathrm{C}_{\mathrm{CH} 4}$ e $\delta^{13} \mathrm{C}_{\mathrm{CO} 2}$ dos gases obtidos (Figura 7) e não indicaram alterações no metabolismo microbial das incubações no intervalo de tempo entre as duas medidas.

A queda das taxas de produção pode também estar associada à diminuição de substratos disponíveis (JONES et al., 2008). A concentração de compostos orgânicos como acetato e hidrocarbonetos utilizados no metabolismo microbial são fatores controladores da produção de metano em sistemas não-convencionais de carvão e de folhelho (GAO et al., 2013; JONES et al., 2008; KRÜGER et al., 2014).

Os testes de troca de headspace e de adição de ácido acético realizados no presente estudo sugerem que a diminuição de substrato disponível pode ter limitado a produção nas incubações testadas. A reinjeção de nitrogênio e a retirada de $\mathrm{CO}_{2}$ e $\mathrm{CH}_{4}$ do headspace não induziram aumento na taxa de produção de $\mathrm{CO}_{2}$ e $\mathrm{CH}_{4}$. A adição de ácido acético no sistema foi responsável pelo reinício da produção e por taxas de produção aproximadamente $57 \%$ 
mais altas do que as observadas nos testes sem adição de ácido acético. Esta característica reforça que os sistemas naturais, onde a recirculação das águas de formação é responsável pela chegada de novos substratos orgânicos adequados à metanogênese, podem apresentar altas taxas de geração de metano. Neste caso, zonas permeáveis para a circulação de água subterrânea, tais como sistemas de fraturas e camadas de arenitos intercaladas a folhelhos, podem elevar a taxa de geração de metano.

Comparações entre amostras incubadas a seco e com adição de água demonstram que a água deionizada funciona como catalizador para a metanogênese, sendo que sua adição foi responsável pelo aumento de $54 \%$ na produção de $\mathrm{CH}_{4}$ e $151 \%$ na produção de $\mathrm{CO}_{2}$. Tal resultado está de acordo com estudos realizados em biorreatores de aterros sanitários, nos quais foram demonstrados os efeitos estimulantes da umidade para comunidades metanogênicas (GURIJALA; SUFLITA, 1993; REINHART; TOWNSEND, 1997). A correlação positiva entre metanogênese e umidade justifica-se no papel da água em reações de hidrólise, transporte de nutrientes, tamponamento e outras importantes funções (REINHART; TOWNSEND, 1997).

Testes ANOVA foram realizados para a comparação da produção total de metano em testes de longo prazo. Unidades geológicas distintas demonstraram diferenças significativas no potencial de produção (ANOVA, F $(2,21)=6,18$; p <0,01). Apesar de relações gerais suscitadas por este trabalho e pela literatura, cada formação pode demonstrar heterogeneidades específicas ligadas às variações do seu contexto geológico como condições de oxidação, histórico térmico e composição química (KOTELNIKOVA, 2002; WHITICAR, 1999).

A Formação Irati demonstrou as maiores taxas de produção de metano para os testes a seco e com adição de água deionizada. Assinaturas pouco usuais de $\delta^{13} \mathrm{C}_{\mathrm{CH} 4}$ and $\delta^{13} \mathrm{C}_{\mathrm{CO} 2}$ indicam que a metanogênese secundária, proveniente da biodegradação de hidrocarbonetos de cadeias mais longas, deve contribuir com a geração biogênica de metano nesta unidade. Em alguns casos específicos, o metano gerado em incubações da Formação Irati demonstraram valores de $\delta^{13} \mathrm{C}$ extremamente pesados em comparação ao esperado para $\mathrm{CH}_{4}$ de origem biogênica (menor que -50\%o) (WHITICAR, 1999). Valores de $\delta^{13} \mathrm{C}_{\mathrm{CH} 4}$ de até $-22 \%$ o foram observados para esta unidade estratigráfica. Embora tais assinaturas isotópicas sejam atípicas para metano de origem biogênica, podem estar associadas a precursores orgânicos enriquecidos em ${ }^{13} \mathrm{C}$ (KOTELNIKOVA, 2002; MARTINI et al., 2003; WHITICAR, 1999). 
Valores de $\delta^{13} \mathrm{C}$ anomalamente positivos para carbonatos são comumente associados a processos metanogênicos (BIRGEL et al., 2015). Interações bioquímicas da redução do $\mathrm{CO}_{2}$ em camadas ricas em material orgânico fornecem $\mathrm{CO}_{2}$ enriquecido em ${ }^{13} \mathrm{C}$ (MARTINI et al., 1998). Consequentemente, a diagênese e a alteração do carbonato nesse ambiente propiciará a formação de minerais com valores mais positivos de $\delta^{13} \mathrm{C}$. Os carbonatos da Formação Irati atingem valores de até $18,3 \%$, indicativos de que a metanogênese já pode ter exercido efeito para o enriquecimento excepcional dos seus minerais (GIOVANI et al., 1974). Valores anômalos de $\delta^{13} \mathrm{C}_{\mathrm{CH} 4}$ e $\delta^{13} \mathrm{C}_{\mathrm{CO} 2}$ obtidos a partir das incubações da Formação Irati demonstram que extrapolações da assinatura isotópica para definir a origem do metano (biogênica versus termogência) estudado estão sujeitas a erro, requerendo abordagens alternativas conforme realizado por Martini et al. (MARTINI et al., 1998).

Considerando todos os testes executados neste estudo, os folhelhos da Formação Ponta Grossa demonstraram o menor potencial para geração de metano em comparação com as outras unidades estudadas. Tal fato pode estar associado ao seu menor conteúdo de COT e maior maturidade térmica, que pode ter eliminado compostos mais reativos do sistema.

A Formação Tremembé, por sua vez, apresentou médias de $\delta^{13} \mathrm{C}_{\mathrm{CH} 4}$ que ultrapassaram $-100 \%$ e médias entre -12 e $-16 \%$ para $\delta^{13} \mathrm{C}$ do $\mathrm{CO}_{2}$ coproduzido. A falta de padrões isotópicos claros dificulta a interpretação da via metabólica principal para as outras unidades estudadas.

Destaca-se ainda, a correlação positiva entre a produção de metano e COT (Figura 12). Tal resultado é condizente com o esperado, uma vez que a matéria orgânica serve como substrato para diversos processos metabólicos de comunidades metanogênicas. A relação obtida por este estudo está de acordo com trabalhos prévios, reportando teores elevados de carbono orgânico como característica importante para potenciais sistemas não-convencionais de metano biogênico (RICE, 1993). 

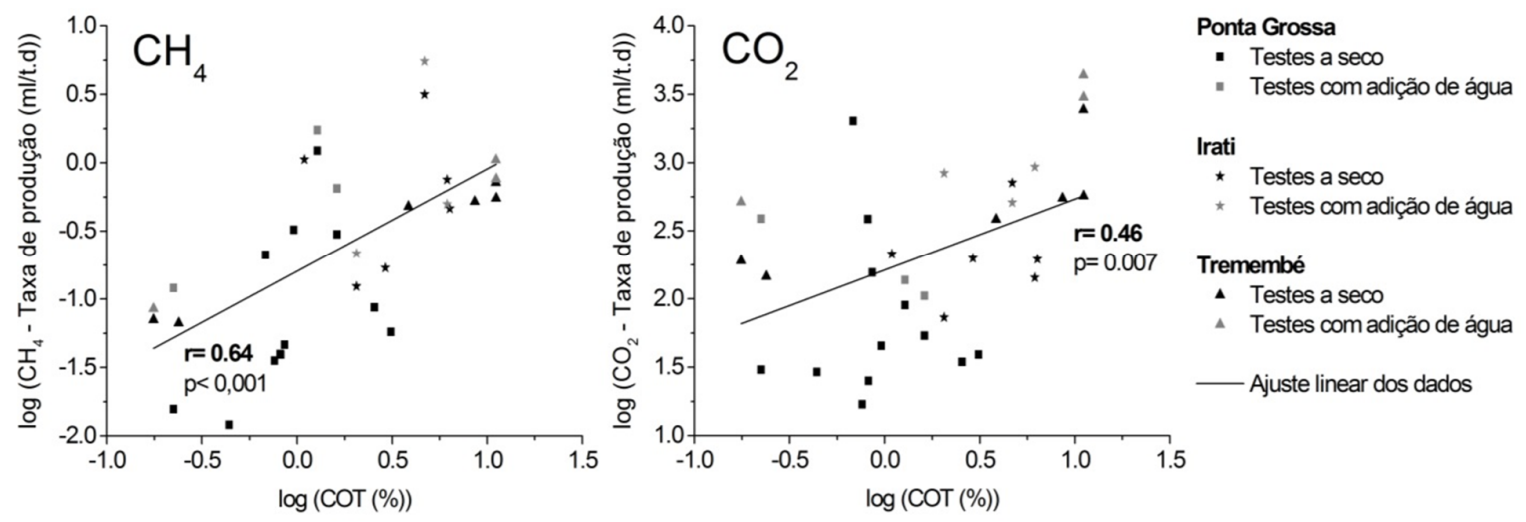

Figura 12 - Comparação entre valores de $\mathrm{COT}$ e taxas de produção de $\mathrm{CH}_{4}$ e $\mathrm{CO}_{2}$ obtidas nos testes de incubação a seco e com adição de água.

A Formação Tremembé demonstrou correlação positiva entre a geração de $\mathrm{CH}_{4} \mathrm{e}$ $\mathrm{CO}_{2}(\mathrm{r}=0,81 ; \mathrm{p}<0,01)$. $\mathrm{O}$ aumento nas taxas de produção de dióxido de carbono são também associados ao maior COT (Figura 12) e menor $\delta^{13} \mathrm{C}_{\mathrm{CH} 4}(\mathrm{r}=-0,77 ; \mathrm{p}=0,025)$. Tal correlação justifica-se pela utilização da matéria orgânica na geração de $\mathrm{CO}_{2}$. A falta de correlação entre $\mathrm{CH}_{4}$ e $\mathrm{CO}_{2}$ para as amostras das formações Ponta Grossa e Irati estão provavelmente associadas às heterogeneidades presentes em seu conteúdo orgânico e sua maturidade térmica mais elevada, uma vez que estas afetam diretamente a disponibilidade de substrato para o metabolismo microbial (KRÜGER et al., 2014; MESLÉ et al., 2013).

\subsection{POTENCIAL PARA PRODUÇÃO DE GÁS NATURAL E CONTRIBUIÇÃO PARA EMISSÃO DE GASES DO EFEITO ESTUFA}

Os resultados obtidos a partir de incubações em série demonstram que a ação de metanógenos em folhelhos ricos em matéria orgânica do sudeste brasileiro podem contribuir de forma significativa para a geração de $\mathrm{CH}_{4}$ e $\mathrm{CO}_{2}$ em diversas condições ambientais (Figura 6). Os folhelhos das formações Ponta Grossa e Irati se estendem por quase toda a Bacia do Paraná com espessura significativa (FERREIRA; CANDIDO; ROSTIROLLA, 2011; SANTOS et al., 2009), e podem gerar reservas expressivas de $\mathrm{CH}_{4}$ e $\mathrm{CO}_{2}$ em escala de tempo geológico (centenas de milhões de anos). A complexidade estrutural promovida pela deformação eocretácea (ZÁLAN et al., 1991) em ambas as unidades também deve contribuir com a concentração do gás gerado. A Formação Tremembé está irregularmente dispersa pela 
área de $3.400 \mathrm{~km}^{2}$ da Bacia de Taubaté com espessura média de 150 m (COGNÉ et al., 2013; RICCOMINI, 1989), fato que a torna reservatório de gás de magnitude inferior, porém com taxas de produção mais elevadas sob algumas condições (adição de ácido acético, por exemplo) e maior proximidade à superfície.

Análises de adsorção de nitrogênio demonstraram que a superfície específica e o volume de poros dos folhelhos estudados apresentam padrões similares aos obtidos em outras unidades de folhelhos produtores de gás natural (Figuras 9 e 13), e indicam, portanto, características adequadas para armazenamento de gás livre. Uma vez que o gás adsorvido é parte fundamental para a avaliação da capacidade total de armazenamento de metano em folhelhos ricos em matéria orgânica, análises de adsorção sob alta pressão em folhelhos das formações Irati, Ponta Grossa e Tremembé são essenciais para melhor avaliação dessas unidades como reservatórios não-convencionais de gás natural (GASPARIK et al., 2012; MILLIKEN; RUDNICKI, 2013). Resultados obtidos por WENIGER et al. (2010) indicam bom potencial para a adsorção de $\mathrm{CH}_{4}$ e $\mathrm{CO}_{2}$ nos folhelhos Permianos e Devonianos da Bacia do Paraná.

Enquanto reservas de metano são importantes do ponto de vista energético, a geração de $\mathrm{CH}_{4}$ e $\mathrm{CO}_{2}$ por comunidades microbiais pode ser de grande relevância para o ciclo global do carbono e mudanças climáticas. Diversos estudos sobre emissões geológicas de metano para a atmosfera detectaram fluxos positivos de metano em interfaces solo-atmosfera relacionados à microdifusão de metano proveniente de sistemas petrolíferos (ETIOPE; KLUSMAN, 2010; ETIOPE; MILKOV; DERBYSHIRE, 2008; ETIOPE, 2004; KLUSMAN; JAKEL, 1998). O metano derivado de reservatórios de hidrocarbonetos e rochas geradoras pode também afetar o potencial de solos para o sequestro de carbono, além de modificar a biogeoquímica de aquíferos adjacentes.

Portanto, ressalta-se que volumes significativos de metano biogênico podem ser gerados em folhelhos ricos em matéria orgânica de bacias do sudeste brasileiro. Entretanto, estudos mais aprofundados são necessários para o estabelecimento da ordem de magnitude em que tais processos afetam o balanço de carbono e o potencial econômico da exploração de gás natural em bacias sedimentares brasileiras. 


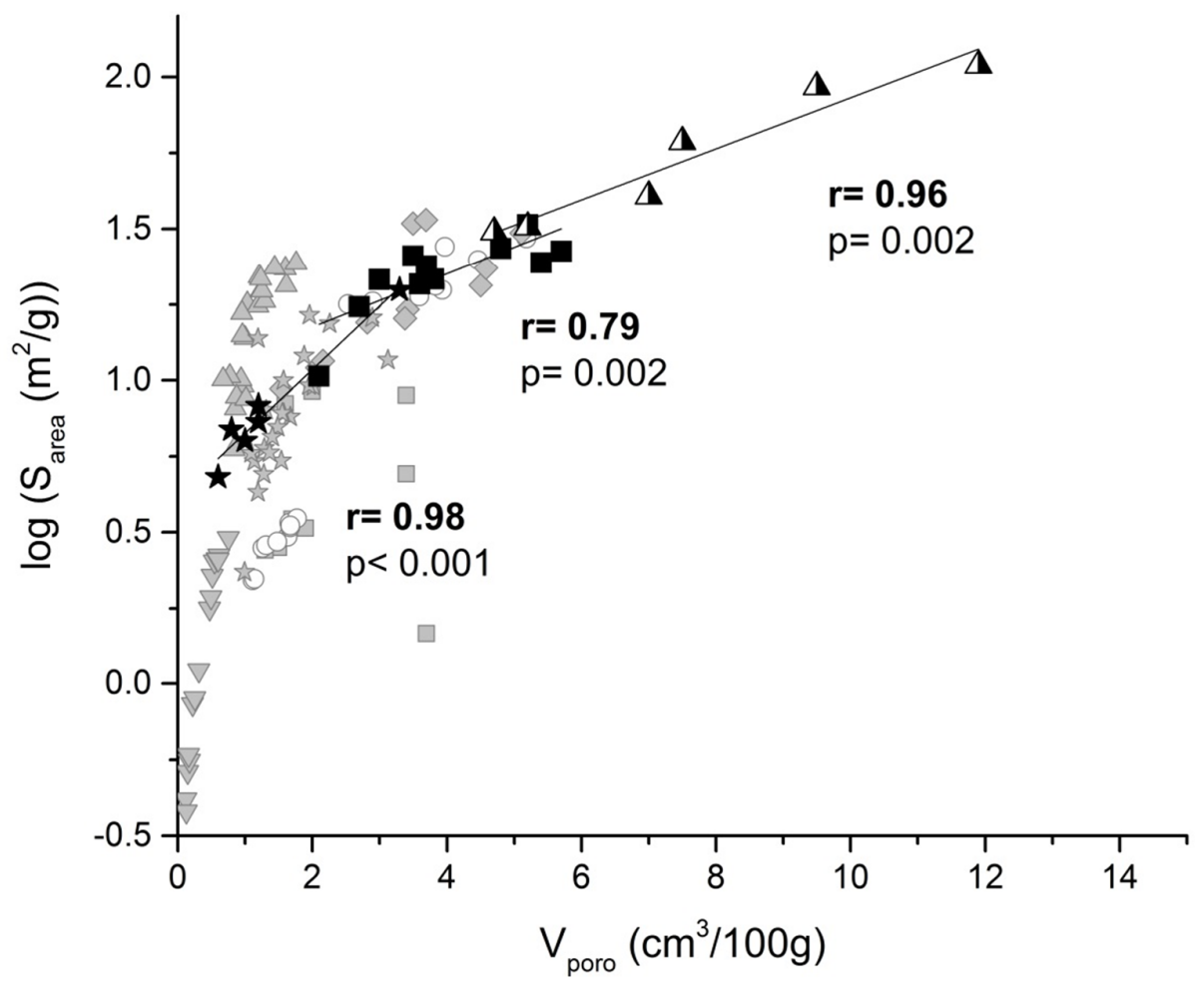

Figura 13 - Comparação entre resultados de adsorção de nitrogênio obtidos neste estudo (símbolos pretos) e dados de trabalhos anteriores (símbolos cinza). Forte correlação positiva é observada entre $V_{\text {poro }}$ e $S_{\text {area }}$ para os folhelhos das formações Ponta Grossa (quadrados pretos),

Irati (estrelas pretas) e Tremembé (triângulos pretos). Dados de estudos anteriores foram obtidos de folhelhos da região do Baixo Yangtze (triângulos cinza; PAN et al., 2015), Bacia de Ordos (quadrados cinza; JI et al., 2014 e triângulos invertidos; LIU et al., 2015), Bacia de Sichuan (losangos; YANG et al., 2014 e círculos; CAO et al., 2015) e da Bacia de Perth e Canning (estrelas cinza; LABANI et al., 2013).

\subsection{INFLUÊNCIA DA ESTRUTURA DE POROS PARA A GERAÇÃO DE $\mathrm{CH}_{4}$}

A forte correlação entre COT, $S_{\text {area }}$ e $V_{\text {poro }}$ obtida por trabalhos anteriores destaca que a matéria orgânica é responsável por acomodar grande parte da porosidade total existente em folhelhos (GASPARIK et al., 2014; LIU; XIONG; LIANG, 2015). Uma vez que COT, $\mathrm{S}_{\text {area, }}$ $\mathrm{V}_{\text {poro }}$ e VEM se interrelacionam, relações de causa e efeito devem ser inferidas com grande atenção. 
Neste contexto, a correlação negativa entre taxas de produção de $\mathrm{CH}_{4} \mathrm{e} \mathrm{V}_{\text {poro }}$ (Figura 10) observada neste trabalho é interpretada de maneira distinta para cada unidade estudada, uma vez que as formações Ponta Grossa, Irati e Tremembé demonstraram diferentes relações entre COT, porosidade e taxas de produção de gás biogênico. Comparações entre a produção de $\mathrm{CH}_{4}$ e $\mathrm{CO}_{2}$ e a estrutura de poros neste estudo descartaram os dados de produção após a adição de ácido acético, com o intuito de reduzir o número de variáveis de controle sobre a produção biogênica.

A correlação negativa entre $\mathrm{CH}_{4}$ e $\mathrm{V}_{\text {poro }}$ para as amostras da Formação Tremembé é produto da forte correlação positiva entre $\mathrm{COT}$ e $\mathrm{CH}_{4}(\mathrm{r}=0,98 ; \mathrm{p}<0,01)$ e forte correlação negativa entre COT e $\mathrm{V}_{\text {poro }}(\mathrm{r}=-0,98 ; \mathrm{p}<0,01)$.

Embora a correlação positiva entre $\mathrm{V}_{\text {poro }}$ e COT seja bem documentada por trabalhos prévios (CHALMERS; ROSS; BUSTIN, 2012; CLARKSON et al., 2013; ROSS; BUSTIN, 2009), tal relação é demonstrada de maneira inversa para os folhelhos da Formação Tremembé. Logo, conclui-se que fatores associados à mineralogia e estrutura interna dos folhelhos devem superar o conteúdo orgânico no controle de variações do $V_{\text {poro. Gasparik et }}$ al. (2012) propõem contribuição significativa dos argilominerais para a capacidade de adsorção de folhelhos com grande teor de esmectita, componente principal dos folhelhos lacustres da Formação Tremembé (GOMES et al., 1996). Adicionalmente, cristais de esmectita possuem maior capacidade de adsorção, são menores e mais frágeis do que os de outros argilominerais predominantes em folhelhos (GASPARIK et al., 2012; JOSH et al., 2012; LAST, 2001). Estas particularidades podem afetar de maneira significativa a estrutura de poro das amostras da Formação Tremembé.

Diferente da Formação Tremembé, a falta de correlação entre COT, $\mathrm{V}_{\text {poro }}$ ou geração de $\mathrm{CH}_{4}$ para amostras das Formações Irati e Ponta Grossa aparenta resultar da presença de hidrocarbonetos de cadeia longa nos poros destas unidades, uma vez que ambos os folhelhos atingiram a janela de geração de petróleo (ARAÚJO et al., 2000; MILANI; FRANÇA; MEDEIROS, 2007; OLIVEIRA, 2012) e apresentaram evidências de entupimento de poros em isotermas de dessorção (Figura 8).

Análises de adsorção conduzidas por Liu et al. (2015) demonstram que $S_{\text {area }}$ possui forte correlação com valores de $\mathrm{V}_{\text {poro }}$, configuração semelhante à obtida no presente trabalho (Figura 13). Esperava-se que a superfície específica pudesse influenciar a produção de gás biogênico ao afetar as taxas de reação e área disponível para ação das comunidades microbiais 
agirem. Entretanto, não se observou tal efeito na geração de $\mathrm{CH}_{4}(\mathrm{r}=-0,52 ; \mathrm{p}<0,01)$ e $\mathrm{CO}_{2}$ $(\mathrm{r}=0,02 ; \mathrm{p}=0,9)$ para as amostras avaliadas.

Além do armazenamento de gás, o tamanho de poro pode influenciar o acesso de comunidades metanogênicas ao substrato, uma vez que estas precisam de cavidade de no mínimo $1 \mu \mathrm{m}$ para prosperar (BOONE; WHITMAN; ROUVIERE, 1993; KOTELNIKOVA, 2002). Grandes volumes de microporos detectados nos folhelhos analisados demonstram que a superfície específica disponível para a ação microbial é limitada. A Figura 13 demonstra o agrupamento de valores de $S_{\text {area }}$ e $V_{\text {poro }}$ de acordo com a unidade estudada. Isto indica que variáveis geológicas associadas à gênese e evolução dos folhelhos, tais como composição mineralógica, presença de compostos orgânicos, grau de compactação e histórico térmico são responsáveis por controles majoritários sobre a porosidade dos folhelhos estudados. Conforme discutido anteriormente, os folhelhos da Formação Tremembé apresentam padrões incomuns e sua composição mineralógica distinta associada ao soterramento raso podem ser responsáveis pelos elevados valores de $\mathrm{V}_{\text {poro }}$ e $S_{\text {area }}$ em comparação com outros exemplos da literatura (CAO et al., 2015; CLARKSON et al., 2013; LABANI et al., 2013; LIU; XIONG; LIANG, 2015; PAN et al., 2015; YANG; NING; LIU, 2014). Os resultados obtidos para as formações Ponta Grossa e Irati, por sua vez, são similares a dados de outros folhelhos Paleozoicos descritos em trabalhos anteriores (Figura 13). 


\section{CONCLUSÕES}

Os folhelhos das formações Irati, Ponta Grossa e Tremembé apresentaram geração significativa de $\mathrm{CH}_{4}$ e $\mathrm{CO}_{2}$. Incubações com a adição de água demonstraram taxa de produção

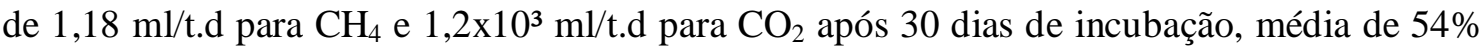
maior para $\mathrm{CH}_{4}$ e $151 \%$ maior para $\mathrm{CO}_{2}$ do que amostras incubadas a seco. Evidencia, portanto, o importante controle exercido pela presença de água na geração de gás biogênico. Logo, alterações climáticas e no nível freático podem desempenhar papel importante para produção biogênica de $\mathrm{CH}_{4}$ e $\mathrm{CO}_{2}$ em folhelhos ricos em matéria orgânica. Razões isotópicas enriquecidas em ${ }^{13} \mathrm{C}$ apresentadas pelo metano gerado a partir de incubações com amostras das formações Irati e Ponta Grossa podem estar relacionadas à biodegradação de hidrocarbonetos, uma vez que tais amostras advêm de unidades com maior maturidade térmica. A adição de ácido acético foi responsável por mudanças significativas nas incubações, as quais apresentaram reinício ou aumento da produção de $\mathrm{CH}_{4}$ e $\mathrm{CO}_{2}$ na maioria dos casos analisados. A renovação do headspace, por sua vez, não demonstrou alterações significativas nos padrões de produção das amostras incubadas.

A Formação Tremembé apresenta maior $V_{\text {poro }}$ e $S_{\text {area }}$ em comparação com outros folhelhos ricos em matéria orgânico, enquanto dados de porosidade e superfície específica obtidos nas formações Irati e Ponta Grossa são similares aos resultados apresentados por estudos da literatura. A correlação negativa entre COT e $V_{\text {poro }}$ para a Formação Tremembé e a falta de correlação para as outras unidades estudadas são explicadas pela elevada proporção de esmectita nas amostras da Formação Tremembé e por possíveis hidrocarbonetos de cadeia longa em poros das formações Irati e Ponta Grossa. Hidrocarbonetos aparentam também alterar os padrões de geração de $\mathrm{CH}_{4}$ e $\mathrm{CO}_{2}$ destas unidades. Tais peculiaridades podem explicar as diferenças observadas para VEM e DTP entre os folhelhos Paleozoicos (formações Ponta Grossa e Irati) e Cenozoicos (Formação Tremembé). Possíveis efeitos da $S_{\text {area }}$ nas taxas de produção de $\mathrm{CH}_{4}$ e $\mathrm{CO}_{2}$ biogênicos não foram identificados.

Cabe ressaltar que extrapolações das taxas de produção obtidas no presente trabalho para as unidades estudadas devem ser consideradas com cautela devido à variabilidade temporal e espacial de fatores geológicos que influenciam a produção biogênica de $\mathrm{CH}_{4} \mathrm{e}$ $\mathrm{CO}_{2}$. Estudos mais aprofundados focando sistemas de metano biogênico em folhelho e a contribuição da geração biogênica às emissões geológicas de $\mathrm{CH}_{4}$ e $\mathrm{CO}_{2}$ são recomendados. 


\section{REFERÊNCIAS BIBLIOGRÁFICAS}

ANSAR, A. et al. Should we build more large dams? The actual costs of hydropower megaproject development. Energy Policy, v. 69, p. 43-56, 2014.

ARAÚJO, L. M. et al. The Atypical Permian Petroleum System of the Paraná Basin, Brazil. AAPG Memoir 73, p. 377-402, 2000.

BIRGEL, D. et al. Methanogenesis produces strong 13C enrichment in stromatolites of Lagoa Salgada, Brazil: A modern analogue for Palaeo-/Neoproterozoic stromatolites? Geobiology, v. 13, n. 3, p. 245-266, 2015.

BOONE, D. R.; WHITMAN, W. B.; ROUVIERE, P. Diversity and taxonomy of methanogens. In: FERRY, J. G. (Ed.). . Methanogenesis. London: Chapman \& Hall, 1993. p. 35-81.

CAO, T. et al. Characterizing the pore structure in the Silurian and Permian shales of the Sichuan Basin, China. Marine and Petroleum Geology, v. 61, 2015.

CHALMERS, G. R. L.; ROSS, D. J. K.; BUSTIN, R. M. Geological controls on matrix permeability of Devonian Gas Shales in the Horn River and Liard basins, northeastern British Columbia, Canada. International Journal of Coal Geology, v. 103, p. 120-131, dez. 2012.

CHEN, J.; XIAO, X. Evolution of nanoporosity in organic-rich shales during thermal maturation. Fuel, v. 129, p. 173-181, ago. 2014.

CLARKSON, C. R. et al. Pore structure characterization of North American shale gas reservoirs using USANS/SANS, gas adsorption, and mercury intrusion. Fuel, v. 103, p. 606616, 2013.

COGNÉ, N. et al. Tectonic setting of the Taubaté Basin (Southeastern Brazil): Insights from regional seismic profiles and outcrop data. Journal of South American Earth Sciences, v. 42, p. 194-204, 2013.

CURTIS, M. E. et al. Development of organic porosity in the Woodford Shale with increasing thermal maturity. International Journal of Coal Geology, v. 103, p. 26-31, dez. 2012.

DANNENBERG, S.; WUDLER, J.; CONRAD, R. Agitation of anoxic paddy soil slurries affects the performance of the methanogenic microbial community. FEMS Microbiology Ecology, v. 22, n. 3, p. 257-263, 1997.

DE FARIA, F. A. M. et al. Estimating greenhouse gas emissions from future Amazonian 
hydroelectric reservoirs. Environmental Research Letters, v. 10, n. 12, 2015.

EIA/ARI. EIA/ARI World Shale Gas and Shale Oil Resource Assessment. 2013.

ETIOPE, G. New directions: GEM—geologic emissions of methane, the missing source in the atmospheric methane budget. Atmospheric Environment, v. 30, n. 2, p. 1094, 2004.

ETIOPE, G.; KLUSMAN, R. W. Geologic emissions of methane to the atmosphere. Chemosphere, v. 49, n. 8, p. 777-789, 2002.

ETIOPE, G.; KLUSMAN, R. W. Methane microseepage in drylands: soil is not always a CH4 sink. Journal of Integrative Environmental Sciences, v. 7, n. sup1, p. 31-38, ago. 2010.

ETIOPE, G.; MILKOV, A. V.; DERBYSHIRE, E. Did geologic emissions of methane play any role in Quaternary climate change? Global and Planetary Change, v. 61, n. 1-2, p. 7988, mar. 2008.

FEARNSIDE, P. M. Impacts of Brazil's Madeira River Dams: Unlearned lessons for hydroelectric development in Amazonia. Environmental Science \& Policy, v. 38, p. 164$172,2014$.

FERREIRA, F.; CANDIDO, A.; ROSTIROLLA, S. P. Correlação gamaespectrométrica de afloramentos e poços: estudo de caso na formação ponta grossa (Bacia do Paraná, Brasil). Brazilian Journal of Geophysics, v. 28, p. 371-396, 2011.

GAO, L. et al. Microbial degradation of sedimentary organic matter associated with shale gas and coalbed methane in eastern Illinois Basin (Indiana), USA. International Journal of Coal Geology, v. 107, p. 152-164, mar. 2013.

GASPARIK, M. et al. High-pressure methane sorption isotherms of black shales from the Netherlands. Energy and Fuels, v. 26, n. 8, p. 4995-5004, 2012.

GASPARIK, M. et al. Geological controls on the methane storage capacity in organic-rich shales. International Journal of Coal Geology, v. 123, p. 34-51, mar. 2014.

GIOVANI, W. DE et al. Unusual isotopic composition of carbonates from the Irati Formation, Brazil. Geological Society of America Bulletin, n. January, p. 1-4, 1974.

GOMES, L. et al. Argilominerais do paleolago Tremembé e sistemas deposicionais relacionados (Paleógeno, Rift Continental do Sudeste do Brasil). Revista Brasileira de Geociências, v. 26, n. 3, p. 167-180, 1996.

GREGG, S. J.; SING., K. S. W. Adsorption Surface Area and Porosity. n. 11, p. 371, 1982. 
GROEN, J. C.; PEFFER, L. A. A.; PÉREZ-RAMÍREZ, J. Pore size determination in modified micro- and mesoporous materials. Pitfalls and limitations in gas adsorption data analysis. Microporous and Mesoporous Materials, v. 60, p. 1-17, 2003.

GURIJALA, K. R.; SUFLITA, J. M. Environmental Factors Influencing Methanogenesis from Refuse in Landfill Samples. Environmental Science \& Technology, v. 27, n. 6, p. 1176-1181, 1993.

HOFFERT, M. I. Climate change. Farewell to fossil fuels? Science, v. 329, n. 5997, p. 12921294, 2010.

HU, H. et al. Experimental investigation of changes in methane adsorption of bitumen-free Woodford Shale with thermal maturation induced by hydrous pyrolysis. Marine and Petroleum Geology, v. 59, p. 114-128, 2015.

JAGIELLO, J.; THOMMES, M. Comparison of DFT characterization methods based on N2, $\mathrm{Ar}, \mathrm{CO} 2$, and $\mathrm{H} 2$ adsorption applied to carbons with various pore size distributions. Carbon, v. 42, n. 7, p. 1225-1229, 2004.

JI, W. et al. Geological controls and estimation algorithms of lacustrine shale gas adsorption capacity: A case study of the Triassic strata in the southeastern Ordos Basin, China. International Journal of Coal Geology, v. 134-135, p. 61-73, 2014.

JONES, E. J. P. et al. Bioassay for estimating the biogenic methane-generating potential of coal samples. International Journal of Coal Geology, v. 76, n. 1-2, p. 138-150, out. 2008.

JOSH, M. et al. Laboratory characterisation of shale properties. Journal of Petroleum Science and Engineering, v. 88-89, p. 107-124, jun. 2012.

KLUSMAN, R.; JAKEL, M. Natural microseepage of methane to the atmosphere from the Denver-Julesburg basin, Colorado. Journal of Geophysical Research, v. 103, 1998.

KOTELNIKOVA, S. Microbial production and oxidation of methane in deep subsurface. Earth-Science Reviews, v. 58, p. 367-395, 2002.

KRÜGER, M. et al. The biogenic methane potential of European gas shale analogues: Results from incubation experiments and thermodynamic modelling. International Journal of Coal Geology, v. 136, p. 59-74, 2014.

KUILA, U. Measurement and Interpretation of Porosity and Pore-size distribution in mudrocks: The Hole Story of Shales. Ph.D. Thesis, p. 238, 2013.

KUILA, U.; PRASAD, M. Aplicattion of nitrogen gas-adsorption technique for 42 
characterization of pore structure of mudrocks. The Leading Edge, v. 32, n. 12, p. 14781485, 2013.

KUUSKRAA, V. et al. World shale gas resources: An initial assessment of 14 regions outside the United States. [s.l: s.n.]. Disponível em: <http://www.madalenaventures.com/download/ARI EIA Intl Gas Shale APR 2011 select.pdf>. Acesso em: 9 jan. 2015.

LABANI, M. M. et al. Evaluation of pore size spectrum of gas shale reservoirs using low pressure nitrogen adsorption, gas expansion and mercury porosimetry: A case study from the Perth and Canning Basins, Western Australia. Journal of Petroleum Science and Engineering, v. 112, p. 7-16, dez. 2013.

LAST, W. M. Mineralogical Analysis of Lake Sediments. Tracking Environmental Change Using Lake Sediments. Volume 2: Physical and Geochemical Methods, v. 2, n. 1991, p. 143-187, 2001.

LAY, J.-J.; YU-YOU, L.; TATSUYA, N. Influences of $\mathrm{pH}$ and moisture content on the methane production in high-solids sludge digestion. Water Research, v. 31, n. 6, p. 15181524, 1997.

LIPPENS, B.; DE BOER, J. H. Studies on pore systems in catalysts V. The t method. Journal of Catalysis, v. 4, n. 3, p. 319-323, 1965.

LIU, X.; XIONG, J.; LIANG, L. Investigation of pore structure and fractal characteristics of organic-rich Yanchang formation shale in central China by nitrogen adsorption/desorption analysis. Journal of Natural Gas Science and Engineering, v. 22, n. April 2011, p. 62-72, 2015.

MARTINI, A. M. et al. Genetic and temporal relations between formation waters and biogenic methane: Upper Devonian Antrim shale, Michigan Basin, USA. Geochimica et Cosmochimica Acta, v. 62, n. 10, p. 1699-1720, 1998.

MARTINI, A. M. et al. Microbial production and modification of gases in sedimentary basins: A geochemical case study from a Devonian shale gas play, Michigan basin. AAPG Bulletin, v. 87, n. 8, p. 1355-1375, ago. 2003.

MELIKOGLU, M. Shale gas: Analysis of its role in the global energy market. Renewable and Sustainable Energy Reviews, v. 37, p. 460-468, set. 2014.

MESLÉ, M. et al. Biostimulation to identify microbial communities involved in methane 
generation in shallow, kerogen-rich shales. Journal of Applied Microbiology, v. 114, n. 1, p. 55-70, jan. 2013.

MILANI, E. J.; FRANÇA, A. B.; MEDEIROS, R. Á. Roteiros Geológicos: Rochas geradoras e rochas-reservatório da Bacia do a Bacia do Paraná, faixa oriental de afloramentos, Estado do Paraná. Boletim de Geociências da Petrobras, p. 135-162, 2007.

MILKOV, A. V. Worldwide distribution and significance of secondary microbial methane formed during petroleum biodegradation in conventional reservoirs. Organic Geochemistry, v. 42, n. 2, p. 184-207, fev. 2011.

MILLIKEN, K.; RUDNICKI, M. Organic matter-hosted pore system, Marcellus Formation (Devonian), Pennsylvania. AAPG Bulletin, v. 2, n. 2, p. 177-200, 2013.

OLIVEIRA, A. F. Paleotemperaturas e paleofluidos da Formação Irati na Borda Leste da Bacia do Paraná: Implicaçoes para a geraçao e migraçao de hidrocarbonetos. Ph.D. Thesis, 2012.

OREMLAND, R.; POLCIN, S. Methanogenesis and sulfate reduction: competitive and noncompetitive substrates in estuarine sediments. Applied and Environmental Microbiology, v. 44, n. 6, p. 1270-1276, 1982.

OSBORN, S. G.; MCINTOSH, J. C. Chemical and isotopic tracers of the contribution of microbial gas in Devonian organic-rich shales and reservoir sandstones, northern Appalachian Basin. Applied Geochemistry, v. 25, n. 3, p. 456-471, mar. 2010.

PACALA, S.; SOCOLOW, R. Stabilization wedges: solving the climate problem for the next 50 years with current technologies. Science, v. 305, n. 5686, p. 968-972, 2004.

PAN, L. et al. A preliminary study on the characterization and controlling factors of porosity and pore structure of the Permian shales in Lower Yangtze region, Eastern China. International Journal of Coal Geology, 2015.

REINHART, D. R.; TOWNSEND, T. G. Landfill bioreactor design \& operation. [s.l.] CRC press, 1997.

RICCOMINI, C. O Rift Continental do Sudeste do Brasil. Ph.D. Thesis, p. 319, 1989.

RICE, D. D. Biogenic gas: Controls, habitats, and resource potential. The Future of Energy Gases. United States Geological Survey Professional Paper, n. 1570, p. 583-606, 1993.

RICE, D. D.; CLAYPOOL, G. E. Generation, Accumulation, and Resource Potential of Biogenic Gas. AAPG Bulletin, v. 65, 1981. 
ROEDER, J. L. What We Learned From the Oil Crisis of 1973: A 30-Year Retrospective. Bulletin of Science, Technology \& Society, v. 25, n. 2, p. 166-169, 2005.

ROSS, D. J. K.; BUSTIN, R. M. The importance of shale composition and pore structure upon gas storage potential of shale gas reservoirs. Marine and Petroleum Geology, v. 26, n. 6, p. 916-927, jun. 2009.

ROUQUEROL, F.; ROUQUEROL, J.; SING, K. Adsorption by Clays , Pillared Layer Structures and Zeolites. Adsorption by Powders and Porous Solids, p. 467, 1999.

ROUQUEROLT, J. et al. Recommendations for the characterization of porous solids. Pure \& Appl. Chem., v. 66, n. 8, p. 1739-1758, 1994.

SABAJ PÉREZ, M. H. Where the Xingu Bends and Will Soon Break. American Scientist, v. 103, n. 6, p. 395, 2015.

SANTOS, R. V. et al. Geochemical and thermal effects of a basic sill on black shales and limestones of the Permian Irati Formation. Journal of South American Earth Sciences, v. 28, n. 1, p. 14-24, 2009.

SCHLEGEL, M. E. et al. Comparison of fluid geochemistry and microbiology of multiple organic-rich reservoirs in the Illinois Basin, USA : Evidence for controls on methanogenesis and microbial transport. Geochimica et Cosmochimica Acta, v. 75, n. 7, p. 1903-1919, 2011 .

SCHOELL, M. The hydrogen and carbon isotopic composition of methane from natural gases of various origins. Geochimica et Cosmochimica Acta, v. 44, 1980.

SHURR, G.; RIDGLEY, J. Unconventional shallow biogenic gas systems. AAPG bulletin, v. 7, n. 3, p. 190-194, 26 jan. 2002.

SING, K. S. W. et al. Reporting physisorption data for gas/solid systems with Special Reference to the Determination of Surface Area and Porosity. Pure \& Appl. Chem., v. 57, n. 4, p. 603-619, 1985.

WANG, Q. et al. Natural gas from shale formation - The evolution, evidences and challenges of shale gas revolution in United States. Renewable and Sustainable Energy Reviews, v. 30, p. 1-28, fev. 2014.

WENIGER, P. et al. High-pressure methane and carbon dioxide sorption on coal and shale samples from the Paraná Basin, Brazil. International Journal of Coal Geology, v. 84, n. 3-4, p. 190-205, dez. 2010. 
WHITICAR, M. Carbon and hydrogen isotope systematics of bacterial formation and oxidation of methane. Chemical Geology, v. 161, n. 1-3, p. 291-314, 1999.

WHITICAR, M.; FABER, E.; SCHOELL, M. Biogenic methane formation in marine and freshwater environments: $\mathrm{CO} 2$ reduction vs. acetate fermentation-Isotope evidence. Geochimica et Cosmochimica Acta, v. 50, n. 5, p. 693-709, 1986.

WOLTERMATE, I.; WHITICAR, M.; SCHOELL, M. Carbon and hydrogen isotopic composition of bacterial methane in a shallow freshwater lake. Limnology and Oceanography, v. 29, n. 5, p. 985-992, 1984.

YANG, F.; NING, Z.; LIU, H. Fractal characteristics of shales from a shale gas reservoir in the Sichuan Basin, China. Fuel, v. 115, p. 378-384, 2014.

ZÁLAN, P. V. et al. Tectonics and sedimentation of the Paraná Basin. Gondwana Seven Proceedings, p. 83-117, 1991.

ZEIKUS, J.; WINFREY, M. Temperature limitation of methanogenesis in aquatic sediments. Applied and Environmental Microbiology, v. 31, n. 1, p. 99-107, 1976.

ZHANG, S. et al. Timing of biogenic gas formation in the eastern Qaidam Basin, NW China. Chemical Geology, v. 352, p. 70-80, 2013.

ZINDER, S. Physiological ecology of methanogens. In: FERRY, J. G. (Ed.). . Methanogenesis. London: Chapman \& Hall, 1993. p. 128-206. 
ANEXO I ARTIGO CIENTİFICO “BIOGENIC METHANE AND CARBON DIOXIDE GENERATION IN ORGANIC-RICH SHALES FROM SOUTHEASTERN BRAZIL” 


\title{
Biogenic methane and carbon dioxide generation in organic-rich shales from southeastern Brazil.
}

Bertassoli Jr. ${ }^{*}$, D. J.; Sawakuchi², H.O.; Almeida ${ }^{1}$, N.S.; Castanheira³, B.; Alem¹, V.A.T.; Camargo'1, M.G.P.; Krusche², A.V.; Brochsztain³, S.; Sawakuchi' ${ }^{1}$, A.O.

*- Corresponding Author, e-mail:dailson.bertassoli@gmail.com

1- Institute of Geosciences, University of São Paulo. Rua do Lago, 562, São Paulo, SP, Brazil - 05508080.

2- Environmental Analysis and Geoprocessing Laboratory, Center for Nuclear Energy in Agriculture, University of São Paulo, Av. Centenário, 303, Piracicaba, SP, Brazil - 13400-970.

3- Federal University of ABC, Rua Santa Adélia, 166, Santo André, SP,Brazil - 09210-170.

\begin{abstract}
Brazilian shale formations are potential biogenic gas systems that are still poorly understood. Organic rich shales from Ponta Grossa (Devonian, Paraná Basin), Irati (Permian, Paraná Basin) and Tremembé (Paleogene, Taubaté Basin) Formations were analyzed in order to evaluate its biogenic methane $\left(\mathrm{CH}_{4}\right)$ production potential and to constrain environmental and geological conditions that may affect methanogenic metabolism. Twenty four shale samples were used for batch incubations in dry and wet conditions and after headspace refilling and acetic acid addition during one year. Phisico-chemical parameters as Total Organic Carbon (TOC), surface area and pore volume were compared to methane production rates in order to understand general geologic controls on production. Biogenic gas production rates in dry tests reached up to $3.17 \mathrm{ml} / \mathrm{t} . \mathrm{d}\left(\mathrm{CH}_{4}\right)$ and $2.45 \times 10^{3} \mathrm{ml} / \mathrm{t} . \mathrm{d}\left(\mathrm{CO}_{2}\right)$ during the first 30 days, with wet samples producing about $54 \%$ faster $\mathrm{CH}_{4}$ and $151 \%$ faster $\mathrm{CO}_{2}$ than dry ones. Headspace refilling did not demonstrate expressive changes on production rates, while acetic acid addition restarted or increased $\mathrm{CH}_{4}$ and $\mathrm{CO}_{2}$ production rates in several microcosms. Irati Formation showed the highest methane production rates for both dry and wet tests, with unusual ranges of $\delta^{13} \mathrm{C}_{\mathrm{CH} 4}$ and $\delta^{13} \mathrm{C}_{\mathrm{CO} 2}$ that may be linked to hydrocarbon biodegradation. Pore volume and specific surface area results varied among shale formations and do not appear to be controlling methanogenic production in studied shale samples.
\end{abstract}




\section{Introduction}

Difficulties to increase hydrocarbon production and restrictions to other energy resource during the past decades fueled the development of shale gas exploration in the U.S. sedimentary basins (Wang et al., 2014). This important turnover is responsible for a global trend of large investments involving potential unconventional petroleum and natural gas systems spread around the world (Melikoglu, 2014).

Despite the global efforts to develop the understanding and characterization of potential shale gas reservoirs as seen in U.S. (Martini et al., 1996), Iran (Aali et al., 2006), Canada (Chalmers et al., 2012), South Africa (Geel et al., 2013) and China (Ju et al., 2014), unconventional shale gas systems in Brazilian onshore sedimentary basins lacks preliminary information, hindering a more effective evaluation of available resources (EIA/ARI, 2013). Meanwhile, according to EIA/ARI (2013) reports, Paraná, Solimões and Parnaíba basins hold together 245 Tcf $\left(6.9\right.$ trillion $\left.\mathrm{m}^{3}\right)$ of technically recoverable shale gas, evidencing a great potential of onshore Brazilian basins for natural gas production. Proximity of consuming market, gas transport and energy transmission infrastructure may highlight the economic potential of Brazilian southeastern basins among others. Additionally, the increase of natural gas share in the Brazilian energy matrix would reduce climate vulnerabilities of hydropower production and the severe social and environmental impacts of hydropower projects in Amazonian rivers (Ansar et al., 2014; Fearnside, 2014; Sabaj Pérez, 2015), which have been considered the major energy to attend the future demand of electricity in Brazil.

The Ponta Grossa, Irati, and Tremembé formations represent important organic rich shales of onshore areas in southern and southeastern Brazil. Devonian (Ponta Grossa Formation) and Permian (Irati Formation) shales are the major hydrocarbon source rocks of the Paraná basin (Milani and Zalan, 1999). Both shale units stand out due to their complex thermal history as a result of heating controlled by burial and by the emplacement of igneous rock bodies (Araújo et al., 2000; Nomura et al., 2014). On the other hand, the Tremembé Formation (Paleogene) includes immature organic rich shale (Mendonça Filho et al., 2010) deposited during the Paleogene. This formation is emplaced in the Taubaté sedimentary basin, which is part of a Cenozoic rift system in Southeastern Brazil (Cogné et al., 2013). Previous efforts to establish the potential of hydrocarbon generation and accumulation in southeastern Brazilian basins focused thermal maturation of organic matter (Araújo et al., 2000; EIA/ARI, 2013). However, biogenic methane $\left(\mathrm{CH}_{4}\right)$ would corresponds to more than $20 \%$ of natural gas accumulations worldwide (Martini et al., 1996; Rice, 1993) and is also a key component on shale gas production rates of many sedimentary basins of North America (Cokar et 
al., 2013; Shurr and Ridgley, 2002). Easy monitoring and inexpensive drilling also contribute to turn biogenic shallow systems (Shurr and Ridgley, 2002) in competitive alternatives for shale gas production, since environmental issues such as difficulties in deep aquifers monitoring may inhibit shale gas exploration in several countries.

Beyond its economic importance, biogenic methane may also contribute to natural geologic greenhouse gases emissions to the atmosphere, which significantly interferes with the global methane budget (Etiope and Klusman, 2002). Therefore, studies focused on the biogenic production potential of methane in organic rich shales will also contribute to improve the understanding on climate change issues.

The present study aims to improve the understanding of biogenic gas generation in three organic rich shales located in Paraná and Taubaté Basins, in Southeastern Brazil. Experiments conducted in this work evaluate the potential biological generation of methane. The characterization of specific surface area and pore size distribution through subcritical nitrogen adsorption techniques and total organic carbon (TOC) content are compared with biogenic gas production data obtained by incubation experiments to understand the influence of shale characteristics on the development of methanogenic communities. Despite the burst of studies based on subcritical $\mathrm{N}_{2}$ adsorption analysis applied to potential shale gas reservoirs, there is a significant gap on the understanding of pore-structure of organic rich shales in Brazilian onshore basins. The use of this technique in the present study provides meaningful information for the evaluation of production and storage potential of the studied shales.

\section{Studied shale formations}

\subsection{Ponta Grossa Formation, Paraná Basin}

Spread over 1.5 million square kilometers, the Paraná Basin has a sedimentary record up to 7 thousand meters thick. Its development during the Devonian period includes sandstones, siltstones and shales that correspond to Ponta Grossa Formation (Milani et al., 2007). Ponta Grossa shales are presented as a source rock of medium quality, with mostly type II kerogen and low hydrogen index. Organic carbon content reaches up to $4 \%$ but is generally between 1.5 and 2.5\% (EIA/ARI, 2013; Milani and Zalan, 1999). Its proven source rocks are in direct contact with the Furnas aquifer, which may be an important catalyst factor for methanogenesis and hydrocarbons biodegradation. Maturation is often related to heat flows from regional thermal anomalies associated to Serra Geral magmatic event, although there are concentric high maturity windows in the deep central basin area (EIA/ARI, 2013). 


\subsection{Irati Formation, Paraná Basin}

The Irati Formation is a Permian unit mainly composed of pyrobetuminous shales, siltstones, dolomites and limestones (Milani et al., 2007). The formation is widespread all over the Paraná Basin with average thickness of $40 \mathrm{~m}$. Irati organic rich shale is characterized by type I kerogen, high hydrogen index and $8 \%$ to $13 \%$ of organic carbon content with peaks reaching up to 24\% (Araújo et al., 2000; EIA/ARI, 2013; Milani et al., 2007). Shale oil from Irati formation attracted investments from the Brazilian government's oil company, which started mining and processing it to extract oil in the 1960s (Nicolini et al., 2011).

Episodes of hydrocarbon generation are linked to the magmatic events of Serra Geral Formation (Araújo et al., 2000). The complex distribution of sills, dykes and flood basalts in the Paraná Basin inputs additional challenges to the understanding of its history of hydrocarbon generation through time (Hurter and Pollack, 1994; Nomura et al., 2014).

\subsection{Tremembé Formation, Taubaté Basin}

The Taubaté Basin is a Paleogene tectonic feature installed in the Continental Rift of Southeastern Brazil (Riccomini, 1989). This depression covers an area of $3.200 \mathrm{~km}^{2}$ and has a maximum thickness of $800 \mathrm{~m}$ of sediments (Cogné et al., 2013; De Carvalho et al., 2011). The Tremembé Formation is a playa-lake lacustrine system of Oligocene age in the Taubaté Basin (Riccomini, 1989). It comprises thermally immature claystones, siltstones and shales reaching a maximum thickness of $400 \mathrm{~m}$. The organic carbon content is generally smaller than $10 \%$, but may vary from $0.18 \%$ to $44 \%$ of predominantly type I kerogen (Mendonça Filho et al., 2010). Organic rich shale of the Tremembé Formation generally exhibit lacustrine algae rich kerogen, with high hydrogen index and low thermal maturity (Bergamaschi et al., 2010; Mendonça Filho et al., 2010); Given its paleoenvironmental favorable conditions for methanogenic communities to grow (e.g. anoxia, low sulfate and high TOC contents; Loureiro and Cardoso, 1990), the Tremembé formation may be considered a potential Early Gas Generation System (Shurr and Ridgley, 2002). 


\section{Methods}

\subsection{Sampling}

Twenty-four shale samples were collected from outcrops of Ponta Grossa (12 samples), Irati (6 samples) and Tremembé (6 samples) formations, located in Paraná and Taubaté basins (Figure 1 and Table 1). The set of shale samples includes specimens with wide spectrum of characteristics (e.g. organic carbon content, age of deposition and thermal maturity).

\subsection{Sample preparation and TOC analysis}

Approximately $300 \mathrm{~g}$ of each shale sample was crushed, milled and sieved under dry conditions. In order to prevent deviations caused by artificial heating, shale samples were dried in laboratory conditions at temperatures below $40^{\circ} \mathrm{C}$. The samples were sieved and fractions finer than $0.25 \mathrm{~mm}$ were used for laboratory procedures as a way to prevent possible effects of grain size on incubation experiments. Samples for TOC analysis were etched with $\mathrm{HCl}$ for elimination of carbonates. TOC quantification was conducted in a Perkin Elmer $2400 \mathrm{CHN}$ Analyzer after pulverization and hydrochloric acid treatment.

\subsection{Incubation experiments and gas $\left(\mathrm{CH}_{4}\right.$ and $\left.\mathrm{CO}_{2}\right)$ analysis}

Incubation experiments were prepared in order to obtain production curves of active microbial consortium inside microcosms. Both $\mathrm{CH}_{4}$ and $\mathrm{CO}_{2}$ production were measured in several environment conditions: dry samples tests; nitrogen refilling tests; long term tests; acetic acid addition tests and wet samples tests.

Sub-samples of shale were powdered and split into triplicates with $15 \mathrm{~g}$ each and disposed inside glass vials $(100 \mathrm{ml})$ sealed with butyl stoppers and aluminum crimps. Flasks were then flushed with $\mathrm{N}_{2}$ to simulate anoxic conditions and stored in dark at 25 ${ }^{\circ} \mathrm{C}$ without agitation to avoid disruption of the methanogenic community (Dannenberg et al., 1997). Sampling was done injecting $5 \mathrm{ml}$ of $\mathrm{N}_{2}$ into the headspace and withdrawing back the same volume of gas. To evaluate the temporal changes in the production rates, analyzes were done 1-5 times per month from January to July 2015.

Final results included dissolution factors to avoid misunderstandings related to nitrogen reinjections during samplings. Concentrations of $\mathrm{CH}_{4}$ and $\mathrm{CO}_{2}$ in the gas samples were analyzed by GC-FID (SHIMADZU GC-17A) coupled with a methanizer. After final measurements, two of the triplicates had the headspace gas removed and submitted to 
$\delta^{13} \mathrm{C}$ isotope analysis on a PICARRO G2201-i Analyzer. The vials were then refilled with $\mathrm{N}_{2}$ to re-start the incubation for a further 27 day period (three samples of each geologic formation were chosen for those extra tests). The third triplicate was maintained as long term control for approximately 1 year.

After this phase, $1 \mathrm{mM}$ of acetic acid dissolved in deionized water $(20 \mathrm{~mL})$ was added into refilled vials in order to study major controls of nutrient availability in $\mathrm{CH}_{4}$ production. Additionally, extra incubations were assembled with fresh samples and deionized water addition $(20 \mathrm{~mL})$. Further measurements were performed following the previous methodology during 49 days for acetic acid addition tests and during 75 days for deionized water addition tests.

Variability in the obtained results is reported as means \pm standard deviation.

\subsection{Subcritical nitrogen adsorption analysis}

Subcritical nitrogen adsorption techniques are useful tools for measuring the influence of the pore-structure of a porous media on fluids flow and mechanical behaviors of shales (Kuila, 2013). Given the importance of micro and mesoporous framework of shale reservoirs, subcritical nitrogen adsorption became an widespread tool for investigation of Pore Size Distribution (PSD) and specific surface area ( $\left.\mathrm{S}_{\text {area }}\right)$ quantification (Chen and Xiao, 2014; Labani et al., 2013; Lu et al., 1995).

Subcritical nitrogen adsorption tests were performed in order to evaluate the PSD and $S_{\text {area }}$ of the incubated samples. Fractions below $0.25 \mathrm{~mm}$ of powdered samples were degassed by vacuum in a Quantachrome NOVA $2200 \mathrm{E}$ device for $24 \mathrm{~h}$ at $55^{\circ} \mathrm{C}$. After preparation, samples were subjected to gas sorption experiments using nitrogen adsorption measurements at $77.3 \mathrm{~K}$ at the same equipment. All recommendations of IUPAC (Sing et al., 1985) were followed during the laboratory procedures.

Surface area calculations were performed at relative pressures below 0.3 in the linear regions of Brunauer, Emmet and Teller (BET) graphic. Total Pore Volume $\left(\mathrm{V}_{\text {pore }}\right)$ was calculated at the highest point of relative pressure, $\mathrm{P} / \mathrm{P}_{0}$ of 0.97 was determined as default. Specific Micropore Volume (SMV) determination was estimated by t-plot technique using the Harkins-Jura thickness equation (Lippens and De Boer, 1965). While Harkins-Jura thickness equation is preferred for mudrock applications, SMV from t-plots for shale samples must be carefully taken as semiquantitative results, useful for comparison among suits of samples (Kuila and Prasad, 2013). Pore diameter distribution was calculated by Density Functional Theory (DFT) and Barret, Joyner and Halenda $(\mathrm{BJH})$ methods. Both methods were complementary used, BJH is a classical 
pore size model widely used for PSD of organic rich shales and DFT provides a more accurate analysis of PSD in micropore scale (Hu et al., 2015; Labani et al., 2013). Pore diameter distribution by BJH method was calculated using the adsorption branch. Points below a $\mathrm{P} / \mathrm{P}_{0}$ of 0.35 were ignored. The choice of adsorption branch is preferred due to Tensile Strength Effects that affects desorption branch and mislead PSD calculations (Groen et al., 2003). $\mathrm{N}_{2}$ adsorption on carbon slit pores at $77.3 \mathrm{~K}$ was used as DFT kernel. Despite the advantages of DFT method, it must be kept in mind that pore structure variations and compositional heterogeneity present in organic rich shales may not completely fit with predictions assumed by the chosen kernel.

\section{Results}

\subsection{Potential production of $\mathrm{CH}_{4}$ and $\mathrm{CO}_{2}$ and $\mathrm{TOC}$ quantification}

A summary of $\mathrm{TOC}$ quantification and rates of $\mathrm{CH}_{4}$ and $\mathrm{CO}_{2}$ production during the first 30 days of the performed experiments are presented in Table 2. The average production rate of the whole set of samples varies according to the tests settings. The total production of $\mathrm{CH}_{4}$ and $\mathrm{CO}_{2}$ during tests is demonstrated in Figure 2. Dry samples tests exhibited mean production rate of $0.44 \pm 0.66 \mathrm{ml} / \mathrm{t} . \mathrm{d}$ for $\mathrm{CH}_{4}$ and $370 \pm 600 \mathrm{ml} / \mathrm{t}$.d for $\mathrm{CO}_{2}$. Biogenic gas generation was significantly lower in nitrogen refilling tests, with mean production of $0.09 \pm 0.12 \mathrm{ml} / \mathrm{t}$.d for $\mathrm{CH}_{4}$ and $490 \pm 910 \mathrm{ml} / \mathrm{t} . \mathrm{d}$ for $\mathrm{CO}_{2}$. Wet samples tests, in turn, demonstrated mean production rates of $1.18 \pm 1.61 \mathrm{ml} / \mathrm{t} . \mathrm{d}$ for $\mathrm{CH}_{4}$ and $1.20 \times 10^{3} \pm 1.40 \times 10^{3} \mathrm{ml} / \mathrm{t} . \mathrm{d}$ for $\mathrm{CO}_{2}$.

TOC quantification showed a high range of results, which varied between $0.18 \%$ and $11.06 \%$. Tremembé samples demonstrated the highest average values and range of TOC $(5.84 \pm 4.66 \%)$, followed by Irati $(3.87 \pm 2.00 \%)$ and Ponta Grossa samples $(1.18 \pm 0.82 \%)$. Methane and carbon dioxide results are detailed below, sorted by tests conditions.

\subsubsection{Dry samples}

Dry sample tests demonstrated heterogeneous findings, with $\mathrm{CH}_{4}$ production varying between $2.50 \mathrm{ml} / \mathrm{t}$ and $111.31 \mathrm{ml} / \mathrm{t}$ during a normalized period of 150 days. Discriminating total production by geologic unit, Ponta Grossa, Irati and Tremembé shales demonstrated average total production of $12.3 \mathrm{ml} / \mathrm{t}, 52.7 \mathrm{ml} / \mathrm{t}$ and $20.8 \mathrm{ml} / \mathrm{t}$, respectively. Large variations occur between samples of the same formation, indicating that important factors determining $\mathrm{CH}_{4}$ production are heterogeneous even within a 
single shale unit. $\mathrm{CH}_{4}$ generation rates in incubations decline from an average of 0.44 $\mathrm{ml} / \mathrm{t}$.d (first 30 days) to $0.16 \mathrm{ml} / \mathrm{t} . \mathrm{d}$ (normalized period of 150 days). Ponta Grossa samples demonstrated average total $\mathrm{CO}_{2}$ production of $23.8 \times 10^{3} \mathrm{ml} / \mathrm{t}$, while Irati and Tremembé showed $12 \times 10^{3} \mathrm{ml} / \mathrm{t}$ and $38.2 \times 10^{3} \mathrm{ml} / \mathrm{t}$, respectively.

Carbon isotope analysis retrieved a wide range of results, $\delta^{13} \mathrm{C}_{\mathrm{CH} 4}$ varied between $-109 \%$ and $-22 \%$ o (-64\%o as mean) and $\delta^{13} \mathrm{C}_{\mathrm{CO}}$ amongst $-30 \%$ and $0 \%$ o $(-18 \%$ as mean). Significant differences were noted through shale formations, as highlighted in Figure 3.

\subsubsection{Headspace refilling}

Subsequent headspace refilling with $\mathrm{N}_{2}$ demonstrated different patterns for $\mathrm{CH}_{4}$ and $\mathrm{CO}_{2}$ results. Just IR-01, IR-02 and TR-02-4 had detectable $\mathrm{CH}_{4}$ from the 9 selected samples, producing an average of $7.8 \mathrm{ml} / \mathrm{t}$ during the 30 days of analysis. $\mathrm{CO}_{2}$, on the other hand, was detected in 5 of 9 incubations. TR-02-2 and TR-02-4 showed the highest $\mathrm{CO}_{2}$ production rates after headspace refilling, with $2.9 \times 10^{3} \mathrm{ml} / \mathrm{t} . \mathrm{d}$ and $1.1 \times 10^{3}$ $\mathrm{ml} / \mathrm{t} . \mathrm{d}$, respectively.

\subsubsection{Long term tests}

Long term tests results were normalized for a 1 year period in order to highlight differences among the three studied formations. Irati shale samples are responsible for the highest $\mathrm{CH}_{4}$ total production, with average of $79.5 \mathrm{ml} / \mathrm{t}$ and a maximum of $152 \mathrm{ml} / \mathrm{t}$ generated by IR-02. The Ponta Grossa Formation demonstrated $17.7 \mathrm{ml} / \mathrm{t}$ as mean production and Tremembé shales returned $35.4 \mathrm{ml} / \mathrm{t}$ as average.

Highest concentrations of carbon dioxide were measured in incubation experiments of Tremembé shale samples. $\mathrm{CO}_{2}$ production rates for the Ponta Grossa, Irati and Tremembé shales were $90.6 \mathrm{ml} / \mathrm{t} . \mathrm{d}, 50.3 \mathrm{ml} / \mathrm{t}$ and $191.4 \mathrm{ml} / \mathrm{t} . \mathrm{d}$, respectively.

$\mathrm{CH}_{4}$ and $\mathrm{CO}_{2}$ generation rates kept declining as in dry sample tests, resulting in production rates nearly $35 \%$ lower for both gases when compared to the first 150 days of incubation.

Small differences of carbon isotope fractionation were recognized when comparing results collected 150 days and 1 year after the beginning of incubation. The average $\delta^{13} \mathrm{C}_{\mathrm{CH} 4}$ for long term tests was $-66 \%$ while $\delta^{13} \mathrm{C}_{\mathrm{CO} 2}$ varied between $-30 \%$ and $4 \%$, with $-17 \%$ as mean. Average results per unit for $\delta^{13} \mathrm{C}_{\mathrm{CH} 4}$ and $\delta^{13} \mathrm{C}_{\mathrm{CO} 2}$ were, respectively, - 
$85 \%$ and $-24 \%$ for Ponta Grossa, $-38 \%$ and $-8 \%$ for Irati and $-91 \%$ and $-12 \%$ for Tremembé formations.

\subsubsection{Acetic acid addition}

Methane and carbon dioxide were measured at days 0, 8, 23 and 49. Total production during 49 days varied between $2.93 \mathrm{ml} / \mathrm{t}$ and $253.3 \mathrm{ml} / \mathrm{t}$. Tremembé samples presented the higher $\mathrm{CH}_{4}$ production, with average of $104.8 \mathrm{ml} / \mathrm{t}(2.14 \mathrm{ml} / \mathrm{t} . \mathrm{d}$ as average production rate). The Irati and Ponta Grossa samples, in turn, produced 51.6 and 5.8 $\mathrm{ml} / \mathrm{t}$ of $\mathrm{CH}_{4} \cdot \mathrm{CO}_{2}$ suffered huge production increase after acetic acid addition, with rates reaching $770 \mathrm{ml} / \mathrm{t} . \mathrm{d}$ for Ponta Grossa, $1.9 \times 10^{3} \mathrm{ml} / \mathrm{t} . \mathrm{d}$ for Irati and $6.22 \times 10^{3} \mathrm{ml} / \mathrm{t} . \mathrm{d}$ for Tremembé samples. The addition of $1 \mathrm{mM}$ of acetic acid dissolved in $20 \mathrm{ml}$ of deionized water appears to be sufficient to improve rates of $\mathrm{CH}_{4}$ and $\mathrm{CO}_{2}$ production, reinforcing the gas generation potential of the studied units in adequate environmental conditions.

Values of $\delta^{13} \mathrm{C}_{\mathrm{CH} 4}$ were available for 3 samples, which varied from $-51 \%$ (TR-02-2) to $-43 \%$ (IR-01). $\mathrm{CO}_{2}$ analysis demonstrated $\delta^{13} \mathrm{C}_{\mathrm{CO} 2}$ varying from $-72 \%$ to $-15 \%$ ( $-34 \%$ o as mean). Isotopic results from $\mathrm{CO}_{2}$ analysis demonstrated average of $-35 \%$ for Ponta Grossa, $-38 \%$ for Irati and $-16 \%$ for Tremembé samples, similar discrepancies were observed in other experimental sets. The heavy values obtained for $\delta^{13} \mathrm{C}_{\mathrm{CH} 4}$ are unexpected, since most of biogenic methane production from acetate fermentation has values below -50\%o (Whiticar et al., 1986). Nevertheless, further studies regarding $\delta D$ and $\delta^{13} \mathrm{C}$ isignatures of biogenic gases may elucidate isotopic anomalies of Brazilian organic rich shales.

\subsubsection{Wet samples}

Incubations under wet conditions exhibited $\mathrm{CH}_{4}$ production reaching up to $179.2 \mathrm{ml} / \mathrm{t}$. Mean production of Ponta Grossa, Tremembé and Irati samples were $30.6 \mathrm{ml} / \mathrm{t}, 68.8$ $\mathrm{ml} / \mathrm{t}$ and $29.8 \mathrm{ml} / \mathrm{t}$, respectively, in a 75 days normalized period. Production rates of wet samples were, on average, $54 \%$ higher than dry ones on the first 30 days of incubation.

As seen in other experimental sets, $\mathrm{CH}_{4}$ generation declined through time, with production averages decreasing from $1.18 \mathrm{ml} / \mathrm{t} . \mathrm{d}$ (30 days production rate) to 0.57 $\mathrm{ml} / \mathrm{t} . \mathrm{d}$ (75 days production rate).

The Tremembé shale demonstrated the highest $\mathrm{CO}_{2}$ production between tested samples, with average rates of $2.9 \times 10^{3} \mathrm{ml} / \mathrm{t} . \mathrm{d}$ during the 75 days of analysis. Irati and 
Ponta Grossa samples produced significantly lower amounts of $\mathrm{CO}_{2}$ in this experiment, with mean production rates $86 \%(400 \mathrm{ml} / \mathrm{t} . \mathrm{d})$ and $95 \%(150 \mathrm{ml} / \mathrm{t} . \mathrm{d})$ lower than Tremembé samples, respectively. Wet samples tests also showed higher $\mathrm{CO}_{2}$ production in comparison with dry tests, with an average increase in production rates of $151 \%$ during the first 30 days.

Wet sample tests demonstrated wide range of carbon isotope fractionation, with $\delta^{13} \mathrm{C}_{\mathrm{CH} 4}$ varying from $-142 \%$ to $-35 \%$ ( $-82 \%$ as mean). Isotopic values of $\delta^{13} \mathrm{C}_{\mathrm{CO} 2}$, in turn, varied between $-27 \%$ and $-13 \%$, with $-19 \%$ as mean. In this case, Tremembé samples showed significant detachment from other incubations, with average $\delta^{13} \mathrm{C}_{\mathrm{CH} 4}$ of $-140 \%$ and $\delta^{13} \mathrm{C}_{\mathrm{CO} 2}-13 \%$. Ponta Grossa and Irati samples, in turn, resulted in average $\delta^{13} \mathrm{C}_{\mathrm{CH} 4}$ of $-48 \%$ and $-58 \%$ and $\delta^{13} \mathrm{C}_{\mathrm{CO} 2}$ of $-23 \%$ and $-17 \%$, respectively.

\subsection{Nitrogen adsorption analysis}

The adsorption/desorption isotherms of selected samples from Ponta Grossa, Irati and Tremembé Formations are demonstrated in Figure 4. According to the International Union of Pure and Applied Chemistry (IUPAC) recommendations, results achieved by this study are a mixture of type II and IV isotherms, with hysteresis loop and high uptake at greater $P / P_{0}$ (Sing et al., 1985). Despite many recent works, which choose only one of the IUPAC physisorption isotherms to fit its results, recommendations from Rouquerol et al. (1999) suggests a new classification (Type Ilb) for this kind of isotherm. Type Ilb isotherms are generally associated to clays and mudrocks, given by slit-shaped pores. It presents an adsorption branch that has similar shape to Type II with a typical $\mathrm{H} 3$ hysteresis loop, without the classic plateau over a range of high $\mathrm{P} / \mathrm{P}_{0}$ presented by Type IV isotherm (Rouquerol et al., 1999). The absence of this plateau indicates a significant volume of macropores, while hysteresis indicates the presence of mesopores (Sing et al., 1985). The forced closure of hysteresis around $P / P_{0}$ of 0.45 for $\mathrm{N}_{2}$ at $77 \mathrm{~K}$ is the result of alterations in pore network promoted by TSE (Gregg and Sing., 1982). Obtained isotherms indicate that Tremembé shales have the most prominent forced closure of hysteresis, suggesting a large volume of pores smaller than $4 \mathrm{~nm}$ (Gregg and Sing., 1982). Irati samples IR-04, IR-03-1 and IR-03-2 show low pressure hysteresis, which is usually associated to swelling or irreversible uptake of adsorbate molecules in micropores (Sing et al., 1985). 


\subsubsection{Specific surface area and pore volume}

Specific surface areas $\left(S_{\text {area }}\right)$ based on multipoint BET method vary between $5 \mathrm{~m}^{2} / \mathrm{g}$ and $109 \mathrm{~m}^{2} / \mathrm{g}$ (Table 3). Ponta Grossa, Irati and Tremembé Formations demonstrated significant differences between their specific surface areas distribution (ANOVA, $F(2,21)=15.95 ; p<0.01)$, showing means of $23 \mathrm{~m}^{2} / \mathrm{g}, 9 \mathrm{~m}^{2} / \mathrm{g}$ and $61 \mathrm{~m}^{2} / \mathrm{g}$, respectively (Figure 5).

For all studied formations, total pore volumes ranged from $0.76 \mathrm{~cm}^{3} / 100 \mathrm{~g}$ to 12.9 $\mathrm{cm}^{3} / 100 \mathrm{~g}$ (Table 3). Significant variation of $V_{\text {pore }}$ was also noted between Formations (ANOVA, $F(2,21)=21.62 ; p<0.01)$. Irati samples showed smaller pore volumes among the other three formations, with $1.6 \mathrm{~cm}^{3} / 100 \mathrm{~g}$ of $\mathrm{V}_{\text {pore }}$ mean and no detectable SMV. The Ponta Grossa Formation has an average of $4.4 \mathrm{~cm}^{3} / 100 \mathrm{~g}$ of $V_{\text {pore }}$, also without detectable SMV and the Tremembé Formation demonstrated an average of 8.3 $\mathrm{cm}^{3} / 100 \mathrm{~g}$ of $V_{\text {pore }}$, with $0.4 \mathrm{~cm}^{3} / 100 \mathrm{~g}$ of SMV (almost $5 \%$ of $V_{\text {pore }}$ ) detected by t-plot (Figure 5). Negative correlation was observed between $\mathrm{CH}_{4}$ production rate and pore volume (Figure 6).

\subsubsection{Pore size distribution: BJH and DFT methods}

In this work, PSD of shale samples from Brazilian southeastern basins were investigated by two complementary methods, BJH and DFT. Table 3 summarizes results collected from both.

Average pore diameter shows significant deviations for BJH (ANOVA, $F(2,21)=5.59$, $\mathrm{p}<0.05)$ and DFT (ANOVA, $F(2,21)=3.82 ; \mathrm{p}<0.05)$ methods. Using $\mathrm{BJH}$ method, average results per unit were $3.34 \pm 0.09 \mathrm{~nm}$ for Ponta Grossa, $3.17 \pm 0.17 \mathrm{~nm}$ for Irati and $3.35 \pm 0.01 \mathrm{~nm}$ for Tremembé formation. DFT method shows average pore diameter of $3.86 \pm 0.09 \mathrm{~nm}$ for Ponta Grossa, $3.46 \pm 0.17 \mathrm{~nm}$ for Irati and $4.15 \pm 0.01$ $\mathrm{nm}$ for Tremembé samples. Both results are significantly lower than data obtained for organic rich shales in previous works, which reach up to $20 \mathrm{~nm}$ in some cases (Cao et al., 2015; Labani et al., 2013; Liu et al., 2015; Pan et al., 2015; Yang et al., 2014).

Graphical representations of PSD data from adsorption branch using BJH method demonstrate wide dispersion between 2 and $40 \mathrm{~nm}$, without any detectable pattern between unimodal, bimodal and multi-modal distributions. PSD histograms composed by DFT data draw attention to differences between two groups of samples. Ponta Grossa and Irati samples demonstrate broader pore size distribution and lower pore volume, while Tremembé samples exhibit distinguishable peaks concentrated in bands bellow $4 \mathrm{~nm}$ (Figure 7). However, quantitative results of micropores volume must be 
carefully considered because of diffusional limitations of nitrogen molecules into carbon micropores (Jagiello and Thommes, 2004; Lay et al., 1997).

\section{Discussion}

\subsection{Factors controlling $\mathrm{CH}_{4}$ and $\mathrm{CO}_{2}$ production}

Biogenic methane in subsurface is mainly generated by two pathways determined by carbon dioxide reduction and acetate fermentation (Rice, 1993). However, formate, alcohols, methylated amides and sulfides are also used as substrates by methanogenic communities (Zinder, 1993).

$\mathrm{CH}_{4}$ production in low sulphate environments often holds a log and a plateau phase, distinguished by a smooth inflection zone possibly associated with changes on substrate consumption and metabolic pathway (Whiticar, 1999). In the absence of competition with sulphate reducing bacteria, methanogenesis by $\mathrm{CO}_{2}$ reduction is less significant in comparison with the acetotrophic pathway. With depletion of acetate, $\mathrm{CO}_{2}$ reduction acquires importance and controls methane production rates (Whiticar, 1999).

As demonstrated by Figure 2, Ponta Grossa and Irati incubation tests exhibit decay trends in biogenic gas generation through time. Tremembé samples, in turn, despite brief production peaks, show a more linear trend for methane production and abrupt production decay for $\mathrm{CO}_{2}$ curves.

Changes in microbial metabolism inside incubations would modify $\delta^{13} \mathrm{C}_{\mathrm{CH} 4}$ and $\delta^{13} \mathrm{C}_{\mathrm{CO} 2}$ signatures, since both metabolic pathways have different fractionation factors (Whiticar, 1999). In the present work, however, comparisons of $\delta^{13} \mathrm{C}_{\mathrm{CH} 4}$ and $\delta^{13} \mathrm{C}_{\mathrm{CO} 2}$ between standard and long term dry tests demonstrate small average differences (Figure 3). Therefore, it does not highlight metabolism changes.

As alternative interpretation, methane production decrease may be explained by substrate depletion (Jones et al., 2008). Bioavailable organic matter is an important limiting factor for methane production in coal and shale systems (Gao et al., 2013; Jones et al., 2008; Krüger et al., 2014).

The possibility of readily metabolizable substrate depletion in incubations is reinforced by nitrogen headspace refilling and acetic acid tests performed in this study. While the first test demonstrated absent or reduced methane and carbon dioxide accumulation, acetic acid addition was responsible for restarting or improving biogenic gas production. Reactivation of methanogenic metabolism provided by acetic acid addition was also responsible for production rates $57 \%$ higher than dry sample tests. This 
feature reinforces that open systems, where recirculation of formation waters are responsible for bringing suitable organic substrates, may present higher production rates.

Comparison between wet and dry tests demonstrates that merely deionized water addition was responsible for substantial improvements in $\mathrm{CH}_{4}$ and $\mathrm{CO}_{2}$ generation in all formations (54\% and $151 \%$, respectively). This result agrees with several studies performed on landfill bioreactors that demonstrated the stimulating effects of moisture on methanogens communities (Gurijala and Suflita, 1993; Reinhart and Townsend, 1997). The positive correlation between methanogenic metabolism and moisture can be explained by the role of water in hydrolysis reactions, nutrients transportation, $\mathrm{pH}$ buffering, surface area exposition and other important functions (Reinhart and Townsend, 1997).

One way ANOVA was performed for comparison of total methane production results obtained in long term tests. Distinct geologic formations demonstrated significant differences for average methane production potential (ANOVA, $F(2,21)=6.18 ; p<0.01$ ). The evaluation of the productive potential of organic rich shales depends on the understanding of intrinsic biogeochemical controls of each studied unit. Despite general relationships raised by this work and previous literature, each formation may demonstrate specific heterogeneities linked to its own features.

Irati Formation demonstrated the highest methane production rates for both dry and wet tests. Unusual ranges of $\delta^{13} \mathrm{C}_{\mathrm{CH} 4}$ and $\delta^{13} \mathrm{C}_{\mathrm{CO} 2}$ consolidate inferences that hydrocarbon biodegradation strongly contributes with biogenic methane generation of Irati samples. Methane generated in Irati incubations presented extremely heavy isotopic fractionations, with $\delta^{13} \mathrm{C}_{\mathrm{CH} 4}$ reaching up to $-22 \%$ in dry sample tests. Although rare, heavy biogenic methane is well documented and a product of environmental stresses and isotopically heavy precursors (Kotelnikova, 2002; Martini et al., 2003).

Unusually positive $\delta^{13} \mathrm{C}$ values for carbonates are commonly associated to methanogenesis processes (Birgel et al., 2015). Biochemical interactions of carbon dioxide reduction in organic rich layers may supply residual heavy $\mathrm{CO}_{2}$ to the system (Martini et al., 1998). Consequently, diagenesis and alteration of associated carbonate in this environment may propitiate highly enriched $\delta^{13} \mathrm{C}$ minerals formation.

Isotopic signature of carbonates from Irati Formation reaches up to $18.3 \%$, indicating that methanogenesis may have already contributed to exceptional isotopic enrichment of unit's $\mathrm{CO}_{2}$ pool (Giovani et al., 1974). Anomalous $\delta^{13} \mathrm{C}$ values obtained for Irati Formation in this work highlight that isotopic extrapolations must be carefully done 
when dealing with gas origin determinations. Further studies concerning organic and inorganic content of studied formations are required for a better understanding of biogenic methane generation processes and isotopic fractionation in this unit.

Considering all performed tests, Ponta Grossa shales demonstrated lower potential for methane generation in comparison with the other studied formations. This fact might be related to its lower TOC availability. Isotopic signatures of $\delta^{13} \mathrm{C}_{\mathrm{CH} 4}$ and $\delta^{13} \mathrm{C}_{\mathrm{CO} 2}$ showed expected means for biogenic methane and carbon dioxide generation.

Tremembé formation showed remarkable negative $\delta^{13} \mathrm{C}_{\mathrm{CH} 4}$ and strong ${ }^{13} \mathrm{C}$ enrichment in coproduced $\mathrm{CO}_{2}$. This possibly indicates that $\mathrm{CO}_{2}$ reduction may prevails over other metabolic pathways, since methane produced by $\mathrm{CO}_{2}$ reduction is generally lighter than products from acetate fermentation (Kotelnikova, 2002; Whiticar et al., 1986). Lack of clear isotopic fractionation patterns precludes further considerations regarding predominant methanogenic pathways for other formations.

Considering wet and dry tests results, positive correlation is observed between methane production and TOC comparisons (Figure 8). This result is expected, since organic matter acts as substrate for the methanogenic communities. The clear relationship obtained in this study agrees with previous works reporting abundant organic matter as important feature for biogenic methane formation (Rice, 1993).

Tremembé formation demonstrates positive correlation between carbon dioxide and methane generation $(r=0.81 ; p<0.01)$. Increases in carbon dioxide production rates are also associated to higher TOC (Figure 8) and lower $\delta^{13} \mathrm{C}_{\mathrm{CH} 4}(r=-0.77 ; p=0.025)$. The positive correlation is also expected, since $\mathrm{CO}_{2}$ production also uses organic matter as substrate.

The lack of clear correlation for Ponta Grossa and Irati samples is probably related to heterogeneities in organic matter composition and thermal maturity, since these directly affect substrate bioavailability (Krüger et al., 2014; Meslé et al., 2013).

\subsection{Implications for natural gas potential and greenhouse emissions}

Results obtained in this work via batch incubations demonstrated that methanogenic consortia in southeastern brazilian shales are able to produce significative quantities of $\mathrm{CH}_{4}$ and $\mathrm{CO}_{2}$ under several environmental conditions (Figure 2). Total production and generation rates from coal and shale incubations of previous works are considerably higher than those obtained in this study. However, several differences in the applied 
methodology inhibits direct comparisons (Jones et al., 2010; Krüger et al., 2014; Papendick et al., 2011; Susilawati et al., 2013).

Ponta Grossa and Irati shales extend throughout most of the Paraná sedimentary basin with expressive thickness (Ferreira et al., 2011; Santos et al., 2009). The large volumes of these formations may overbalance low production rates, and the structural complexity induced by Serra Geral magmatism in both formations may also contribute to concentrate biogenic gas generated through long periods. The Tremembé Formation, however, is irregularly dispersed through the $3.400 \mathrm{~km}^{2}$ of Taubate Basin, with a standard thickness of 150 m (Cogné et al., 2013; Riccomini, 1989). Lower volumes may reduce the significance of this shale as gas reservoir.

Nitrogen adsorption analysis demonstrated that specific surface area and pore volume of the studied shales presents similar patterns to other prolific formations, indicating a good potential for free gas storage (Figure 9). As adsorbed gas is an important part of total gas storage capacity in organic rich shales (Gasparik et al., 2012; Milliken and Rudnicki, 2013), high-pressure adsorption analyses in Irati, Ponta Grossa and Tremembé samples are essential for a better evaluation of these units as unconventional reservoirs. Results obtained by Weniger et al. (2010) indicate good potential for $\mathrm{CH}_{4}$ and $\mathrm{CO}_{2}$ in Permian and Devonian shales of Paraná basin.

While these potential reservoirs of biogenic $\mathrm{CH}_{4}$ may be important from an economic perspective, it represents a potential source of greenhouse gas to the atmosphere. Large emissions of $\mathrm{CH}_{4}$ from hydrocarbon prolific basins through the soils were already detected in several places (Etiope and Klusman, 2010; Etiope, 2004; Etiope et al., 2008; Klusman and Jakel, 1998). Methane from hydrocarbon reservoirs and source rocks may also affect the potential of soils as sink and the groundwater chemistry. The present study highlights the potential of biogenic methane generated from organic rich shales to the methane budget inside Brazilian sedimentary basins. Nevertheless, further studies regarding the potential of southeastern Brazilian shale units as greenhouse gases source to atmosphere or unconventional biogenic systems must be developed in order to understand the magnitude of these features and generate a baseline of potential emissions prior to extraction by fracking.

\subsection{The influence of pore characteristics on $\mathrm{CH}_{4}$ production}

Results obtained by previous works demonstrate a strong positive correlation between TOC, $S_{\text {area }}$ and $V_{\text {pore, }}$, highlighting the strong control of organic-associated porosity in total porosity of organic rich shales (Gasparik et al., 2014; Liu et al., 2015). Since TOC, 
$\mathrm{S}_{\text {area}}, \mathrm{V}_{\text {pore }}$ and micropore volume ratio $\left(\mathrm{M}_{\text {ratio }}\right)$ may have interrelated influences, relationships of cause and effect must be carefully inferred. The unexpected negative correlation between $\mathrm{CH}_{4}$ production rates and pore volume (Figure 6) may have different explanations for distinct units, since Ponta Grossa, Irati and Tremembé samples demonstrate varied patterns of relationship among TOC, porosity and biogenic gas production rates. Comparisons among gas productivity and pore structure ignore acetic addition data in order to reduce the number of variables affecting biogenic production.

Negative correlation of $\mathrm{CH}_{4}$ production and $\mathrm{V}_{\text {pore }}$ for Tremembé samples is a collateral effect of the strong positive correlation between $\mathrm{TOC} \times \mathrm{CH}_{4}$ production $(r=0.98 ; p<0.01)$ and strong negative correlation of TOC $\times V_{\text {pore }}(r=-0.98 ; p<0.01)$.

Although positive correlation between $\mathrm{V}_{\text {pore }}$ and TOC content is well documented by previous works (Chalmers et al., 2012; Clarkson et al., 2013; Ross and Bustin, 2009), the opposite relationship is seen for Tremembé shales. Therefore, it is possible to state that other geologic controls such as mineralogy and fabric are outweighing TOC impacts on $\mathrm{V}_{\text {pore. }}$. Gasparik et al. (2012) reported significant contribution of clay minerals to sorption capacities in samples with high contents of smectite, which is the main component of Tremembé's lacustrine sediments (Gomes et al., 1996). Smectite particles have the highest sorption capacity, are smaller and weaker than other major clay mineral groups (Gasparik et al., 2012; Josh et al., 2012; Last, 2001). These particularities may strongly affect the pore structure and require further investigations regarding its effects in Tremembé shales.

Differently from Tremembé, the lack of correlation between TOC and $\mathrm{V}_{\text {pore }}$ or $\mathrm{CH}_{4}$ production for Ponta Grossa and Irati samples may result from the influence of impregnated hydrocarbons affecting the pore structure in these units. Both shales reached oil generation windows in several intervals (EIA/ARI, 2013) and showed evidences of pore clogging in desorption isotherms.

Subcritical nitrogen adsorption analyses conducted by Liu et al. (2015) demonstrate that $S_{\text {area }}$ is strongly dependent on $V_{\text {pore }}$ values, the same configuration observed in data obtained by this work (Figure 9). Specific surface area may influence biogenic gas production by affecting reaction rates. However, the strong correlation between pore volume and $\mathrm{S}_{\text {area }}$ conceals minor effects of specific surface area on reaction rates and minimizes its possible effects on $\mathrm{CH}_{4}(r=-0.52 ; p<0.01)$ and $\mathrm{CO}_{2}(r=0.02 ; p=0.9)$ production. 
Despite limitations (Kuila and Prasad, 2013), analysis of $\mathrm{M}_{\text {ratio }}$ demonstrates negative correlation with TOC for Tremembé and Ponta Grossa units. As discussed above, the same controls affecting $V_{\text {pore }}$ and $S_{\text {area }}$ may be responsible for these correlation patterns.

Pore size may influence the gas storage potential and the accessibility of the methanogenic consortium to substrate. It is also an important feature for methanogenic communities, which require an average space of $1 \mu \mathrm{m}$ to thrive (Boone et al., 1993; Kotelnikova, 2002). High contents of small pores detected in studied shales limit the surface area that is available to methanogens to interact with.

Despite variations inward shale units, Figure 9 demonstrates clustering of surface area and pore volume data. As clustering of $\mathrm{V}_{\text {pore }}$ data is sorted by geologic formation, it is reasonable to conclude that geologic particularities like mineralogy, organic geochemistry, fabric and thermal history are responsible for major controls affecting that feature. As discussed above, Tremembé shales have abnormal patterns regarding geologic controls over pore volume. Distinct mineralogy and fabric associated to a brief burial history may elucidate the remarkably high $\mathrm{V}_{\text {pore }}$ for this unit in comparison with other examples (Cao et al., 2015; Clarkson et al., 2013; Labani et al., 2013; Liu et al., 2015; Pan et al., 2015; Yang et al., 2014). Those features are also responsible for $S_{\text {area }}$ controls, given its dependence and strong correlation with total pore volume. Figure 9 also demonstrates that Ponta Grossa and Irati results are consistent with previous data from Paleozoic organic rich shales.

\section{Conclusions}

The present study identified significant biogenic production of $\mathrm{CH}_{4}$ and $\mathrm{CO}_{2}$ in three Brazilian shale formations. Wet shales produced about $54 \%$ more $\mathrm{CH}_{4}$ and $151 \%$ more $\mathrm{CO}_{2}$ than dry shales, highlighting the importance of water in methanogenic communities. Thus, paleoclimate and water table changes would play an important role for $\mathrm{CH}_{4}$ and $\mathrm{CO}_{2}$ production within shales. Heavy isotopic signatures of $\delta^{13} \mathrm{CH}_{4}$ for Irati and Ponta Grossa Formations might be related to secondary microbial methane or mixture with thermogenic methane, since these units experienced the generation of heavier hydrocarbon due to heating under burial. Acetic acid addition was responsible for improvement or restart of biogenic methane generation in microcosms environment. Headspace refilling, however, did not demonstrated significant changes in $\mathrm{CH}_{4}$ and $\mathrm{CO}_{2}$ production. 
The Tremembé Formation presents larger pore volume and higher specific surface in comparison with other organic rich shales, while Ponta Grossa and Irati shales are similar to results collected by previous studies from the literature. The unexpected relationships between TOC and $\mathrm{V}_{\text {pore }}$ are explained by high contents of smectite in Tremembé samples and impregnated bitumen in Irati and Ponta Grossa samples. This pattern is probably related to the differences of $M_{\text {ratio }}$ and PSD among the studied Paleozoic and Cenozoic shales. Strong influences of substrate availability on microbial metabolism may inhibit possible effects of specific surface area on reaction rates during biogenic methane generation.

Extrapolations of $\mathrm{CH}_{4}$ and $\mathrm{CO}_{2}$ production rates towards geologic formations are hindered by geologic and geochemical variability in subsurface. Future studies regarding the impact of biogenic generation to geologic emissions of $\mathrm{CH}_{4}$ and $\mathrm{CO}_{2}$ in Brazilian sedimentary basins are recommended.

\section{Aknowledgements}

We acknowledge Petrobras for the financial support provided through the Human Resources Formation Program in Petroleum and Environmental Geology (PFRH-241) and CNPq (grant 454362/2014-5). We thank Alexandra Montebello, Maria Pelissari and Laura Furukawa for the assistance in laboratory procedures. 


\section{References}

Aali, J., Rahimpour-Bonab, H., Kamali, M.R., 2006. Geochemistry and origin of the world's largest gas field from Persian Gulf, Iran. J. Pet. Sci. Eng. 50, 161-175. doi:10.1016/j.petrol.2005.12.004

Ansar, A., Flyvbjerg, B., Budzier, A., Lunn, D., 2014. Should we build more large dams? The actual costs of hydropower megaproject development. Energy Policy 69, 43-56. doi:10.1016/j.enpol.2013.10.069

Araújo, L.M., Trigüis, J.A., Cerqueira, J.R., Freitas, L.C.S., 2000. The Atypical Permian Petroleum System of the Paraná Basin, Brazil. AAPG Mem. 73 377-402.

Bergamaschi, S., Rodrigues, R., Pereira, E., 2010. Oil shale from the Tremembé Formation, Taubaté Basin, Brazil. Search Discov. 80080, 10pp.

Birgel, D., Meister, P., Lundberg, R., Horath, T.D., Bontognali, T.R.R., Bahniuk, A.M., de Rezende, C.E., Vasconcelos, C., McKenzie, J.A., 2015. Methanogenesis produces strong $13 \mathrm{C}$ enrichment in stromatolites of Lagoa Salgada, Brazil: a modern analogue for Palaeo/Neoproterozoic stromatolites? Geobiology. doi:10.1111/gbi.12130

Boone, D.R., Whitman, W.B., Rouviere, P., 1993. Diversity and taxonomy of methanogens, in: Ferry, J.G. (Ed.), Methanogenesis. Chapman \& Hall, London, pp. 35-81.

Cao, T., Song, Z., Wang, S., Cao, X., Li, Y., Xia, J., 2015. Characterizing the pore structure in the Silurian and Permian shales of the Sichuan Basin, China. Mar. Pet. Geol. 61. doi:10.1016/j.marpetgeo.2014.12.007

Chalmers, G.R.L., Ross, D.J.K., Bustin, R.M., 2012. Geological controls on matrix permeability of Devonian Gas Shales in the Horn River and Liard basins, northeastern British Columbia, Canada. Int. J. Coal Geol. 103, 120-131. doi:10.1016/j.coal.2012.05.006

Chen, J., Xiao, X., 2014. Evolution of nanoporosity in organic-rich shales during thermal maturation. Fuel 129, 173-181. doi:10.1016/j.fuel.2014.03.058

Clarkson, C.R., Solano, N., Bustin, R.M., Bustin, a. M.M., Chalmers, G.R.L., He, L., Melnichenko, Y.B., Radliński, a. P., Blach, T.P., 2013. Pore structure characterization of North American shale gas reservoirs using USANS/SANS, gas adsorption, and mercury intrusion. Fuel 103, 606-616. doi:10.1016/j.fuel.2012.06.119

Cogné, N., Cobbold, P.R., Riccomini, C., Gallagher, K., 2013. Tectonic setting of the Taubaté Basin (Southeastern Brazil): Insights from regional seismic profiles and outcrop data. J. South Am. Earth Sci. 42, 194-204. doi:10.1016/j.jsames.2012.09.011

Cokar, M., Ford, B., Kallos, M.S., Gates, I.D., 2013. New gas material balance to quantify biogenic gas generation rates from shallow organic-matter-rich shales. Fuel 104, 443-451. doi:10.1016/j.fuel.2012.06.054

Dannenberg, S., Wudler, J., Conrad, R., 1997. Agitation of anoxic paddy soil slurries affects the performance of the methanogenic microbial community. FEMS Microbiol. Ecol. 22, 257-263. doi:10.1016/S0168-6496(96)00097-9

De Carvalho, A.M.A., Vidal, A.C., Kiang, C.H., 2011. Delimitação do Embasamento da Bacia de Taubaté. Geol. USP - Ser. Cient. 11, 19-32.

EIA/ARI, 2013. EIA/ARI World Shale Gas and Shale Oil Resource Assessment.

Etiope, G., 2004. New directions: GEM-geologic emissions of methane, the missing source in the atmospheric methane budget. Atmos. Environ. 30, 1094. 
Etiope, G., Klusman, R.W., 2002. Geologic emissions of methane to the atmosphere. Chemosphere 49, 777-789.

Etiope, G., Klusman, R.W., 2010. Methane microseepage in drylands: soil is not always a CH4 sink. J. Integr. Environ. Sci. 7, 31-38. doi:10.1080/19438151003621359

Etiope, G., Milkov, A. V., Derbyshire, E., 2008. Did geologic emissions of methane play any role in Quaternary climate change? Glob. Planet. Change 61, 79-88. doi:10.1016/j.gloplacha.2007.08.008

Fearnside, P.M., 2014. Impacts of Brazil's Madeira River Dams: Unlearned lessons for hydroelectric development in Amazonia. Environ. Sci. Policy 38, 164-172. doi:10.1016/j.envsci.2013.11.004

Ferreira, F., Candido, A., Rostirolla, S.P., 2011. Correlação gamaespectrométrica de afloramentos e poços: estudo de caso na formação ponta grossa (Bacia do Paraná, Brasil). Brazilian J. Geophys. 28, 371-396.

Gao, L., Brassell, S.C., Mastalerz, M., Schimmelmann, A., 2013. Microbial degradation of sedimentary organic matter associated with shale gas and coalbed methane in eastern Illinois Basin (Indiana), USA. Int. J. Coal Geol. 107, 152-164. doi:10.1016/j.coal.2012.09.002

Gasparik, M., Bertier, P., Gensterblum, Y., Ghanizadeh, A., Krooss, B.M., Littke, R., 2014. Geological controls on the methane storage capacity in organic-rich shales. Int. J. Coal Geol. 123, 34-51. doi:10.1016/j.coal.2013.06.010

Gasparik, M., Ghanizadeh, a., Bertier, P., Gensterblum, Y., Bouw, S., Krooss, B.M., 2012. High-pressure methane sorption isotherms of black shales from the Netherlands. Energy and Fuels 26, 4995-5004. doi:10.1021/ef300405g

Geel, C., Schulz, H.M., Booth, P., De Wit, M., Horsfield, B., 2013. Shale gas characteristics of Permian black shales in South Africa: Results from recent drilling in the Ecca Group (Eastern Cape). Energy Procedia 40, 256-265. doi:10.1016/j.egypro.2013.08.030

Giovani, W. De, Salati, E., Marini, J.O., Friedman, I., 1974. Unusual isotopic composition of carbonates from the Irati Formation, Brazil. Geol. Soc. Am. Bull. 14.

Gomes, L., Anna, S., Neto, M. a X.B., Riccomini, C., Coimbra, a M., Sant'Anna, L.G., Brandt Neto, M., Valarelli, J. V, 1996. Argilominerais do paleolago Tremembé e sistemas deposicionais relacionados (Paleógeno, Rift Continental do Sudeste do Brasil). Rev. Bras. Geociências 26, 167-180.

Gregg, S.J., Sing., K.S.W., 1982. Adsorption Surface Area and Porosity 371.

Groen, J.C., Peffer, L.A.A., Pérez-Ramírez, J., 2003. Pore size determination in modified micro- and mesoporous materials. Pitfalls and limitations in gas adsorption data analysis. Microporous Mesoporous Mater. 60, 1-17. doi:10.1016/S1387-1811(03)00339-1

Gurijala, K.R., Suflita, J.M., 1993. Environmental Factors Influencing Methanogenesis from Refuse in Landfill Samples. Environ. Sci. Technol. 27, 1176-1181. doi:10.1021/es00043a018

Hu, H., Zhang, T., Wiggins-Camacho, J.D., Ellis, G.S., Lewan, M.D., Zhang, X., 2015. Experimental investigation of changes in methane adsorption of bitumen-free Woodford Shale with thermal maturation induced by hydrous pyrolysis. Mar. Pet. Geol. 59, 114-128. doi:10.1016/j.marpetgeo.2014.07.029

Hurter, S.J., Pollack, H.N., 1994. Effect of the Cretaceous Serra Geral igneous event on the temperatures and heat flow of the Paraná Basin, Southern Brazil. Basin 
Res. 6, 239-244. doi:10.1111/j.1365-2117.1994.tb00087.x

Jagiello, J., Thommes, M., 2004. Comparison of DFT characterization methods based on $\mathrm{N} 2, \mathrm{Ar}, \mathrm{CO} 2$, and $\mathrm{H} 2$ adsorption applied to carbons with various pore size distributions. Carbon N. Y. 42, 1225-1229. doi:10.1016/j.carbon.2004.01.022

Ji, W., Song, Y., Jiang, Z., Wang, X., Bai, Y., Xing, J., 2014. Geological controls and estimation algorithms of lacustrine shale gas adsorption capacity: A case study of the Triassic strata in the southeastern Ordos Basin, China. Int. J. Coal Geol. 134135, 61-73. doi:10.1016/j.coal.2014.09.005

Jones, E.J.P., Voytek, M. a, Corum, M.D., Orem, W.H., 2010. Stimulation of methane generation from nonproductive coal by addition of nutrients or a microbial consortium. Appl. Environ. Microbiol. 76. doi:10.1128/AEM.00728-10

Jones, E.J.P., Voytek, M. a., Warwick, P.D., Corum, M.D., Cohn, A., Bunnell, J.E., Clark, A.C., Orem, W.H., 2008. Bioassay for estimating the biogenic methanegenerating potential of coal samples. Int. J. Coal Geol. 76, 138-150. doi:10.1016/j.coal.2008.05.011

Josh, M., Esteban, L., Delle Piane, C., Sarout, J., Dewhurst, D.N., Clennell, M.B., 2012. Laboratory characterisation of shale properties. J. Pet. Sci. Eng. 88-89, 107-124. doi:10.1016/j.petrol.2012.01.023

Ju, Y., Wang, G., Bu, H., Li, Q., Yan, Z., 2014. China organic-rich shale geologic features and special shale gas production issues. J. Rock Mech. Geotech. Eng. 6, 196-207. doi:10.1016/j.jrmge.2014.03.002

Klusman, R., Jakel, M., 1998. Natural microseepage of methane to the atmosphere from the Denver-Julesburg basin, Colorado. J. Geophys. Res. 103.

Kotelnikova, S., 2002. Microbial production and oxidation of methane in deep subsurface. Earth-Science Rev. 58, 367-395.

Krüger, M., van Berk, W., Arning, E.T., Jiménez, N., Schovsbo, N.H., Straaten, N., Schulz, H.-M., 2014. The biogenic methane potential of European gas shale analogues: Results from incubation experiments and thermodynamic modelling. Int. J. Coal Geol. 136, 59-74. doi:10.1016/j.coal.2014.09.012

Kuila, U., 2013. Measurement and Interpretation of Porosity and Pore-size distribution in mudrocks: The Hole Story of Shales. Ph.D. Thesis 238.

Kuila, U., Prasad, M., 2013. Aplicattion of nitrogen gas-adsorption technique for characterization of pore structure of mudrocks. Lead. Edge 32, 1478-1485.

Labani, M.M., Rezaee, R., Saeedi, A., Hinai, A. Al, 2013. Evaluation of pore size spectrum of gas shale reservoirs using low pressure nitrogen adsorption, gas expansion and mercury porosimetry: A case study from the Perth and Canning Basins, Western Australia. J. Pet. Sci. Eng. 112, 7-16. doi:10.1016/j.petrol.2013.11.022

Last, W.M., 2001. Mineralogical Analysis of Lake Sediments. Track. Environ. Chang. Using Lake Sediments. Vol. 2 Phys. Geochemical Methods 2, 143-187.

Lay, J.-J., Yu-You, L., Tatsuya, N., 1997. Influences of pH and moisture content on the methane production in high-solids sludge digestion. Water Res. 31, 1518-1524.

Lippens, B., De Boer, J.H., 1965. Studies on pore systems in catalysts $\mathrm{V}$. The $\mathrm{t}$ method. J. Catal. 4, 319-323. doi:10.1016/0021-9517(65)90307-6

Liu, X., Xiong, J., Liang, L., 2015. Investigation of pore structure and fractal characteristics of organic-rich Yanchang formation shale in central China by nitrogen adsorption/desorption analysis. J. Nat. Gas Sci. Eng. 22, 62-72. doi:10.1016/j.jngse.2014.11.020 
Loureiro, M.R.B., Cardoso, J.N., 1990. Aromatic hydrocarbons in the Paraiba Valley oil shale. Org. Geochem. 15, 351-359. doi:10.1016/0146-6380(90)90161-R

Lu, X., Li, F., Watson, A., 1995. Identifying and Estimating Desorption From Devonian Shale Gas Production Data, in: 64th Annual Technical Conference and Exhibition of the Society of Petroleum Engineers.

Martini, A., Budai, J., Walter, L., Schoell, M., 1996. Microbial generation of economic accumulations of methane within a shallow organic-rich shale. Nature.

Martini, A.M., Walter, L.M., Budat, J.M., Ku, T.C.W., Kaiser, C.J., Schoell, M., 1998. Genetic and temporal relations between formation waters and biogenic methane: Upper Devonian Antrim shale, Michigan Basin, USA. Geochim. Cosmochim. Acta $62,1699-1720$.

Martini, A.M., Walter, L.M., Ku, T.C.W., Budai, J.M., McIntosh, J.C., Schoell, M., 2003. Microbial production and modification of gases in sedimentary basins: A geochemical case study from a Devonian shale gas play, Michigan basin. Am. Assoc. Pet. Geol. Bull. 87, 1355-1375. doi:10.1306/031903200184

Melikoglu, M., 2014. Shale gas: Analysis of its role in the global energy market. Renew. Sustain. Energy Rev. 37, 460-468. doi:10.1016/j.rser.2014.05.002

Mendonça Filho, G.M., Chagas, R.B. a, Menezes, T.R., Mendonça, J.O., da Silva, F.S., Sabadini-Santos, E., 2010. Organic facies of the Oligocene lacustrine system in the Cenozoic Taubaté basin, Southern Brazil. Int. J. Coal Geol. 84, 166-178. doi:10.1016/j.coal.2010.07.004

Meslé, M., Périot, C., Dromart, G., Oger, P., 2013. Biostimulation to identify microbial communities involved in methane generation in shallow, kerogen-rich shales. J. Appl. Microbiol. 114, 55-70. doi:10.1111/jam.12015

Milani, E.J., Henrique, J., Melo, G. De, Souza, P.A. De, Fernandes, L.A., França, A.B., 2007. Bacia do Paraná. Bol. Geociências da Petrobras 15, 265-287.

Milani, E.J., Zalan, P.V., 1999. An Outline of the Geology and Petroleum Systems of the Paleozoic Interior Basins of South America. Episodes - Newsmag. Int. Union Geol. Sci. 7.

Milliken, K., Rudnicki, M., 2013. Organic matter-hosted pore system, Marcellus Formation (Devonian), Pennsylvania. Am. Assoc. Pet. Geol. Bull. 2, 177-200. doi:10.1306/07231212048

Nicolini, J., Pereira, B., Pillon, C., Machado, V., Lopes, W., de Andrade, J., Mangrich, A., 2011. Characterization of Brazilian oil shale byproducts planned for use as soil conditioners for food and agro-energy production. J. Anal. Appl. Pyrolysis 90, 112-117. doi:10.1016/j.jaap.2010.11.001

Nomura, S.F., Sawakuchi, a. O., Bello, R.M.S., Méndez-Duque, J., Fuzikawa, K., Giannini, P.C.F., Dantas, M.S.S., 2014. Paleotemperatures and paleofluids recorded in fluid inclusions from calcite veins from the northern flank of the Ponta Grossa dyke swarm: Implications for hydrocarbon generation and migration in the Paraná Basin. Mar. Pet. Geol. 52, 107-124. doi:10.1016/j.marpetgeo.2014.01.010

Pan, L., Xiao, X., Tian, H., Zhou, Q., Chen, J., Li, T., Wei, Q., 2015. A preliminary study on the characterization and controlling factors of porosity and pore structure of the Permian shales in Lower Yangtze region, Eastern China. Int. J. Coal Geol. doi:10.1016/j.coal.2015.05.005

Papendick, S.L., Downs, K.R., Vo, K.D., Hamilton, S.K., Dawson, G.K.W., Golding, S.D., Gilcrease, P.C., 2011. Biogenic methane potential for Surat Basin , Queensland coal seams. Int. J. Coal Geol. 88, 123-134. doi:10.1016/j.coal.2011.09.005 
Reinhart, D.R., Townsend, T.G., 1997. Landfill bioreactor design \& operation. CRC press.

Riccomini, C., 1989. O Rift Continental do Sudeste do Brasil. Ph.D. Thesis 319.

Rice, D.D., 1993. Biogenic gas: Controls, habitats, and resource potential. Futur. Energy Gases. United States Geol. Surv. Prof. Pap. 583-606. doi:10.1016/03603199(95)90005-5

Ross, D.J.K., Bustin, R.M., 2009. The importance of shale composition and pore structure upon gas storage potential of shale gas reservoirs. Mar. Pet. Geol. 26, 916-927. doi:10.1016/j.marpetgeo.2008.06.004

Rouquerol, F., Rouquerol, J., Sing, K., 1999. Adsorption by Clays , Pillared Layer Structures and Zeolites. Adsorpt. by Powders Porous Solids 467.

Sabaj Pérez, M.H., 2015. Where the Xingu Bends and Will Soon Break. Am. Sci. 103, 395. doi:10.1511/2015.117.395

Santos, R.V., Dantas, E.L., Oliveira, C.G. De, José, C., Alvarenga, S. De, Wense, C., Guimarães, E.M., Oliveira, F.B., 2009. Geochemical and thermal effects of a basic sill on black shales and limestones of the Permian Irati Formation. J. South Am. Earth Sci. 28, 14-24. doi:10.1016/j.jsames.2008.12.002

Shurr, G., Ridgley, J., 2002. Unconventional shallow biogenic gas systems. Am. Assoc. Pet. Geol. Bull. 7, 190-194. doi:10.1038/ngeo2069

Sing, K.S.W., Everett, D.H., Haul, R. a. W., Moscou, L., Pierotti, R. a., Rouquérol, J., Siemieniewska, T., 1985. Reporting physisorption data for gas/solid systems with Special Reference to the Determination of Surface Area and Porosity. Pure Appl. Chem. 57, 603-619. doi:10.1351/pac198557040603

Susilawati, R., Papendick, S.L., Gilcrease, P.C., Esterle, J.S., Golding, S.D., Mares, T.E., 2013. Preliminary investigation of biogenic gas production in Indonesian low rank coals and implications for a renewable energy source. J. Asian Earth Sci. 77, 234-242. doi:10.1016/j.jseaes.2013.08.024

Wang, Q., Chen, X., Jha, A.N., Rogers, H., 2014. Natural gas from shale formation The evolution, evidences and challenges of shale gas revolution in United States. Renew. Sustain. Energy Rev. 30, 1-28. doi:10.1016/j.rser.2013.08.065

Weniger, P., Kalkreuth, W., Busch, A., Krooss, B.M., 2010. High-pressure methane and carbon dioxide sorption on coal and shale samples from the Paraná Basin, Brazil. Int. J. Coal Geol. 84, 190-205. doi:10.1016/j.coal.2010.08.003

Whiticar, M., 1999. Carbon and hydrogen isotope systematics of bacterial formation and oxidation of methane. Chem. Geol. 161, 291-314.

Whiticar, M., Faber, E., Schoell, M., 1986. Biogenic methane formation in marine and freshwater environments: $\mathrm{CO} 2$ reduction vs. acetate fermentation-Isotope evidence. Geochim. Cosmochim. Acta 50, 693-709. doi:10.1016/00167037(86)90346-7

Yang, F., Ning, Z., Liu, H., 2014. Fractal characteristics of shales from a shale gas reservoir in the Sichuan Basin, China. Fuel 115, 378-384. doi:10.1016/j.fuel.2013.07.040

Zinder, S., 1993. Physiological ecology of methanogens, in: Ferry, J.G. (Ed.), Methanogenesis. Chapman \& Hall, London, pp. 128-206. 


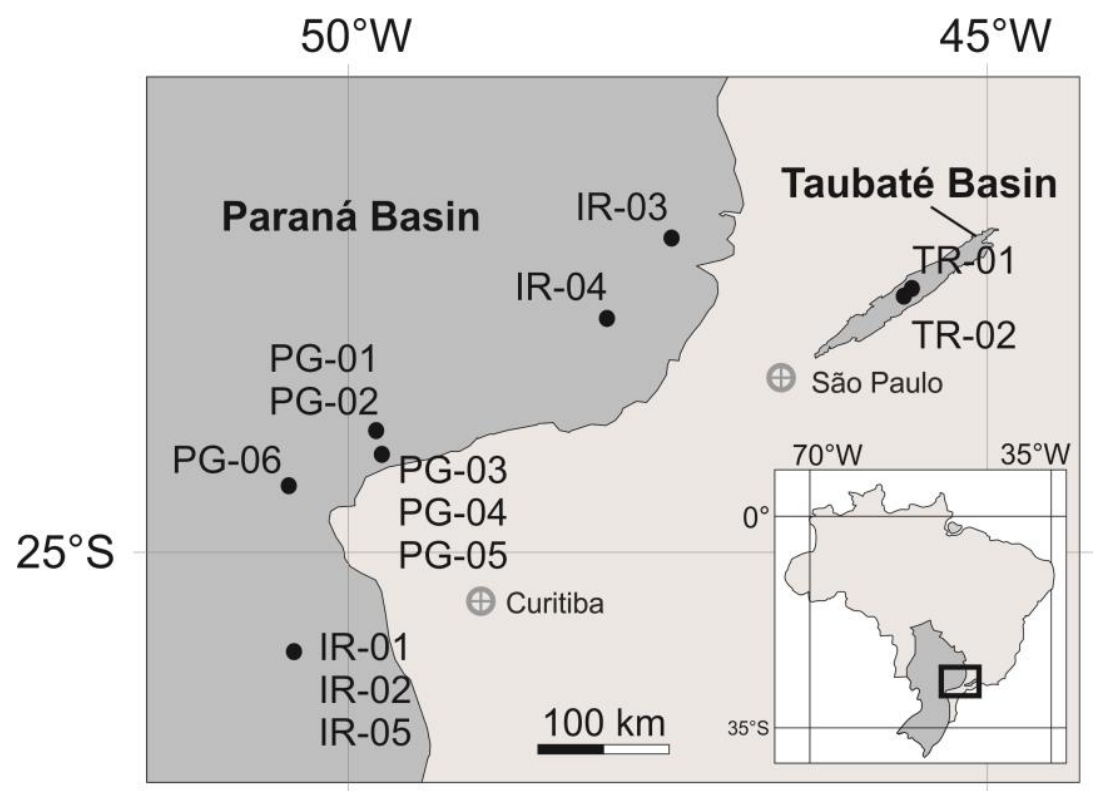

Figure 1 - Location of sampling sites in the Paraná and Taubaté basins, southeastern Brazil. 


\section{$\mathrm{CH}_{4}$ Production Curves}

\section{$\mathrm{CO}_{2}$ Production Curves}
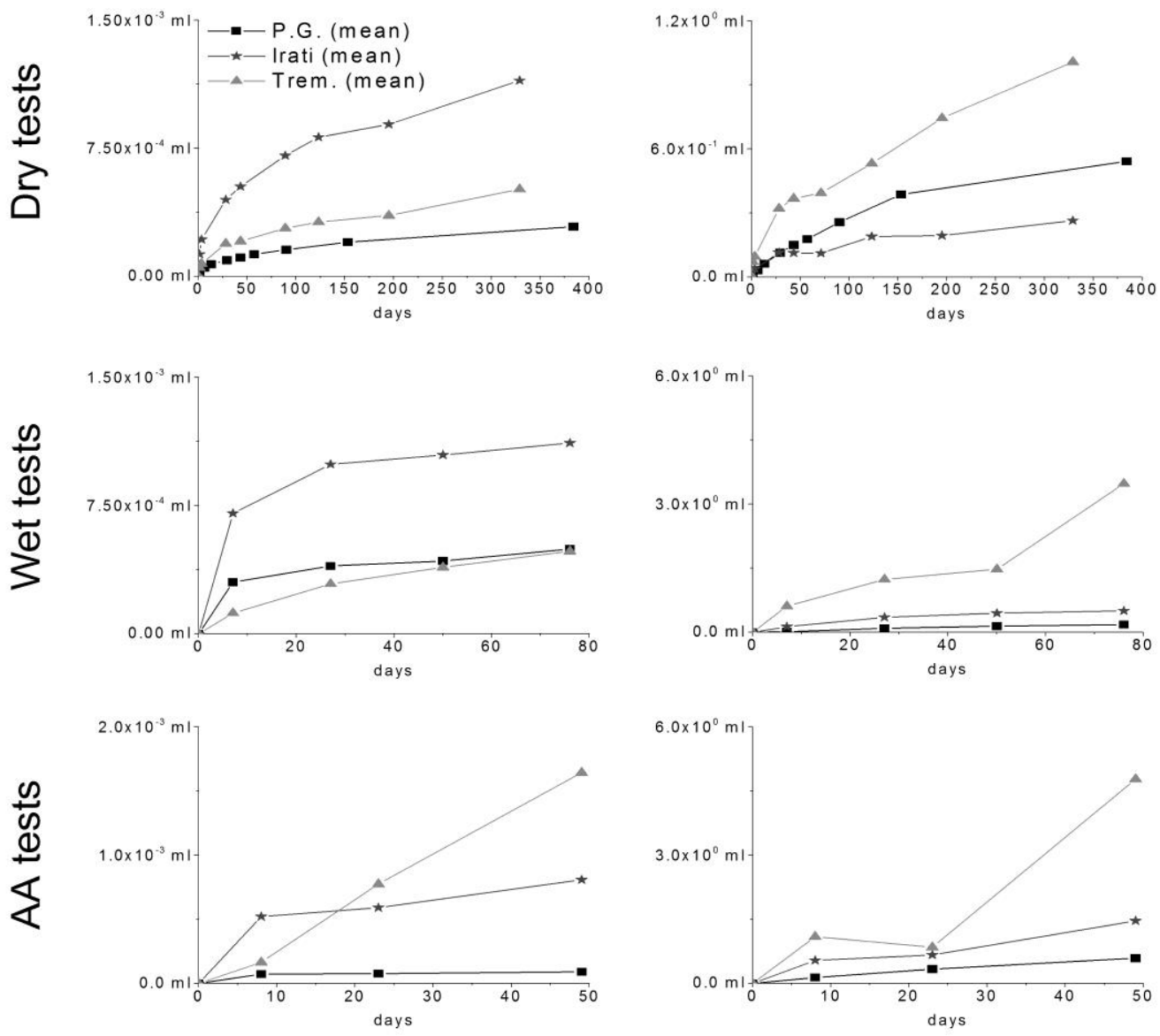

Figure $2-\mathrm{CH}_{4}$ and $\mathrm{CO}_{2}$ accumulation curves obtained for incubation tests using samples of Ponta Grossa (P.G.), Irati and Tremembé (Trem.) Formations. Tests were performed under dry and (Dry tests) wet (Wet tests) conditions and after acetic acid addition (AA tests).
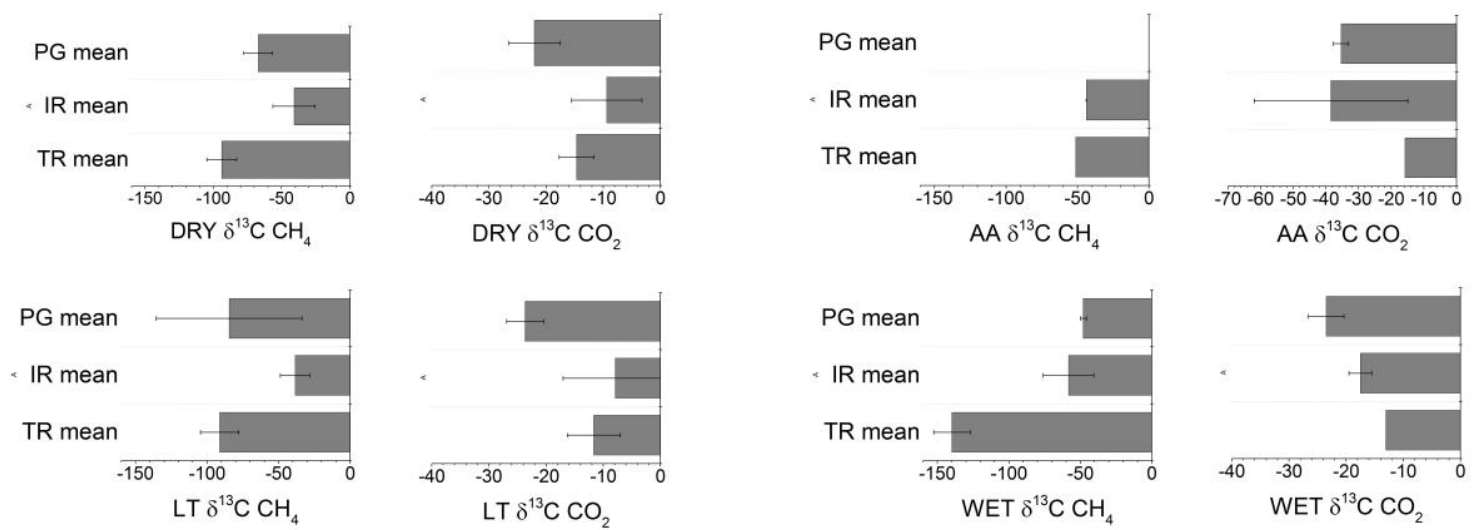

Figure 3 - Carbon isotope $\left(\delta^{13} \mathrm{C}\right)$ results for $\mathrm{CH}_{4}$ and $\mathrm{CO}_{2}$. Produced in dry sample tests (DRY), long term tests (LT), acetic acid addition tests (AA) and wet sample tests (WET). 

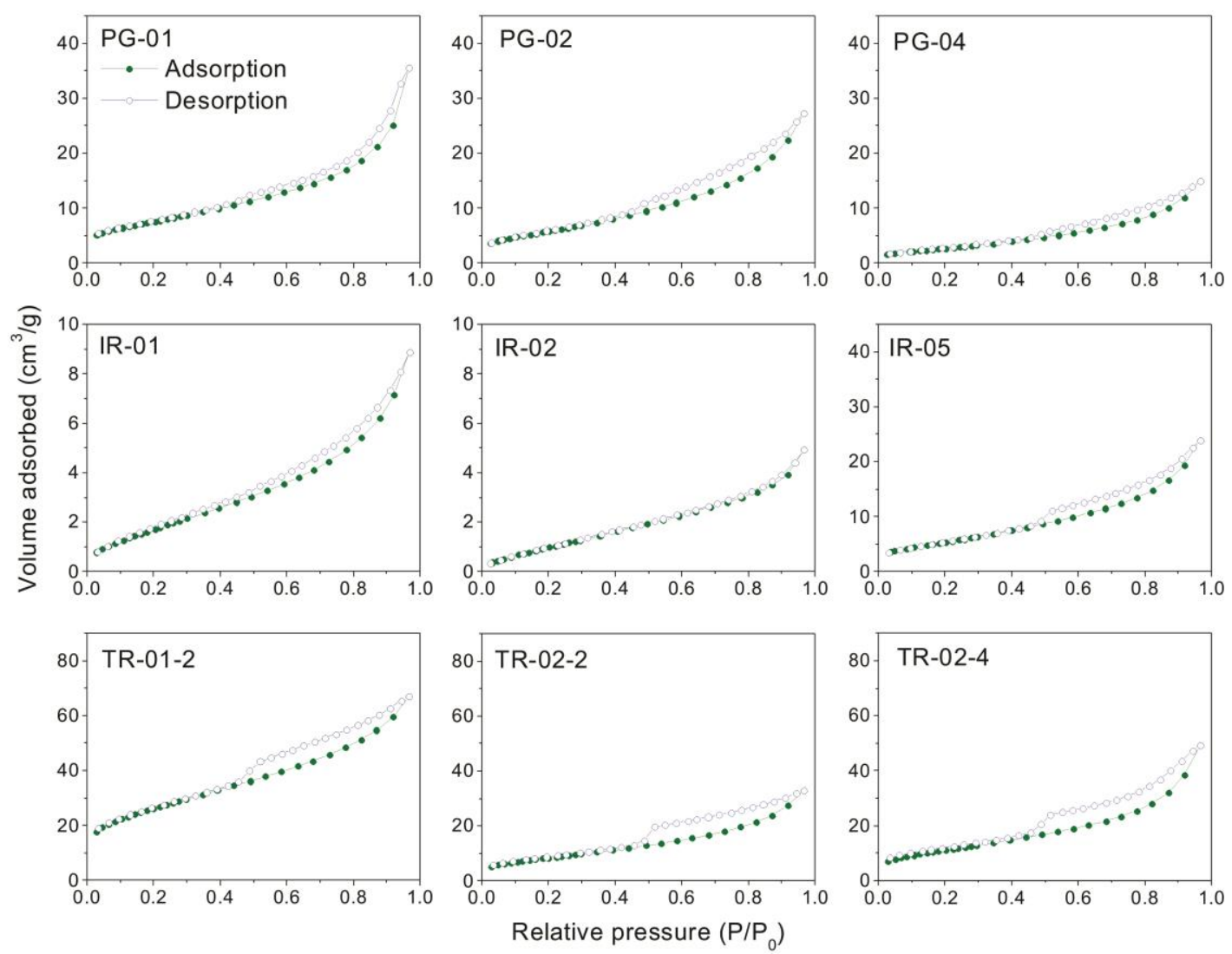

Figure 4 - Isotherms from subcritical nitrogen adsorption analysis of samples for the Ponta Grossa (PG), Irati (IR) and Tremembé (TR) Formations. Three samples of each unit were selected in order to summarize the full spectrum of the studied units.

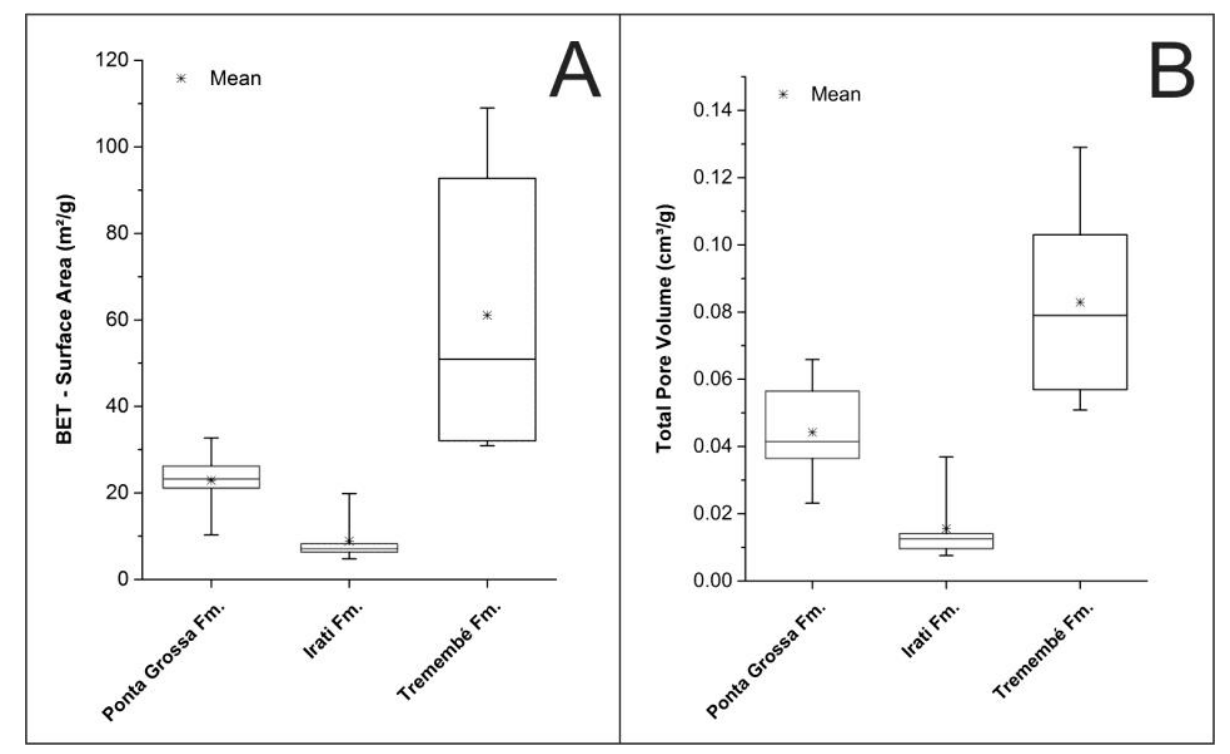

Figure 5 - Boxplots of surface area results from BET analysis $(A, n=24)$ and total pore volume $(B, n=24)$ calculated at the highest point of $P / P_{0}$. 


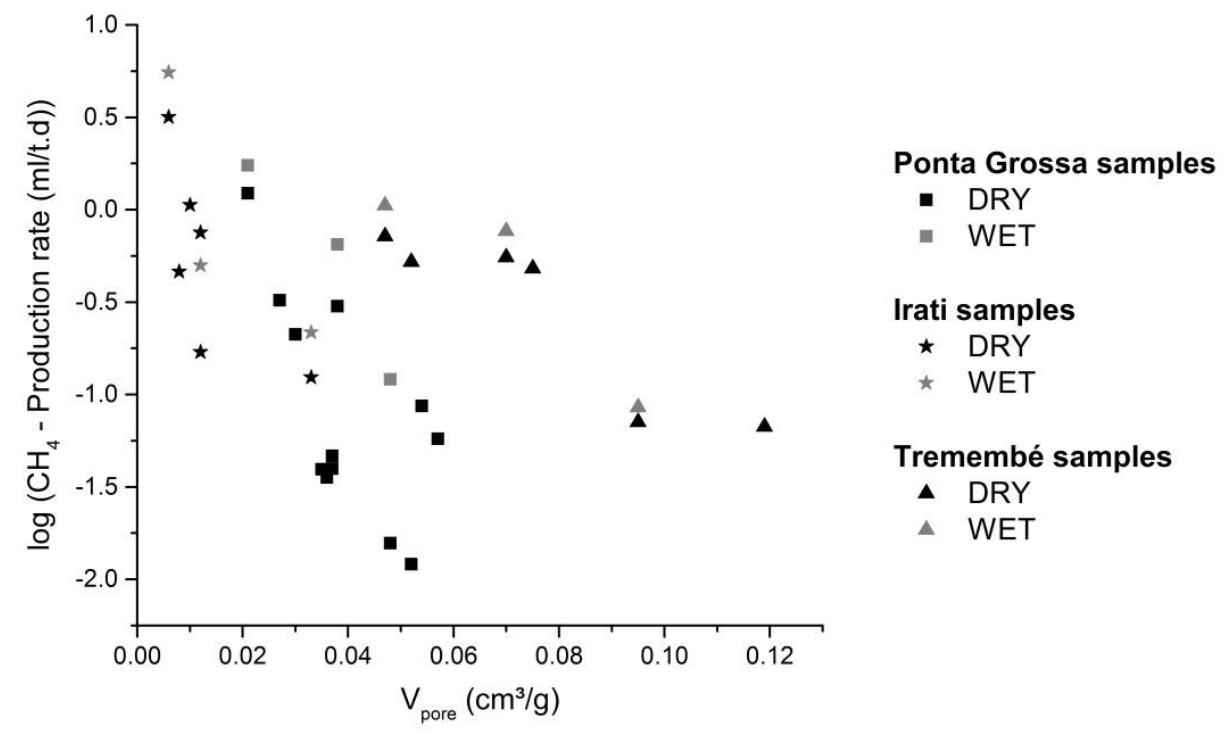

Figure 6 - Relationship between $\mathrm{CH}_{4}$ production and pore volume $(\mathrm{r}=-0.42 ; \mathrm{p}=0.01)$ for samples of Ponta Grossa, Irati and Tremembé Formations. Data obtained for production experiments under dry and wet conditions.
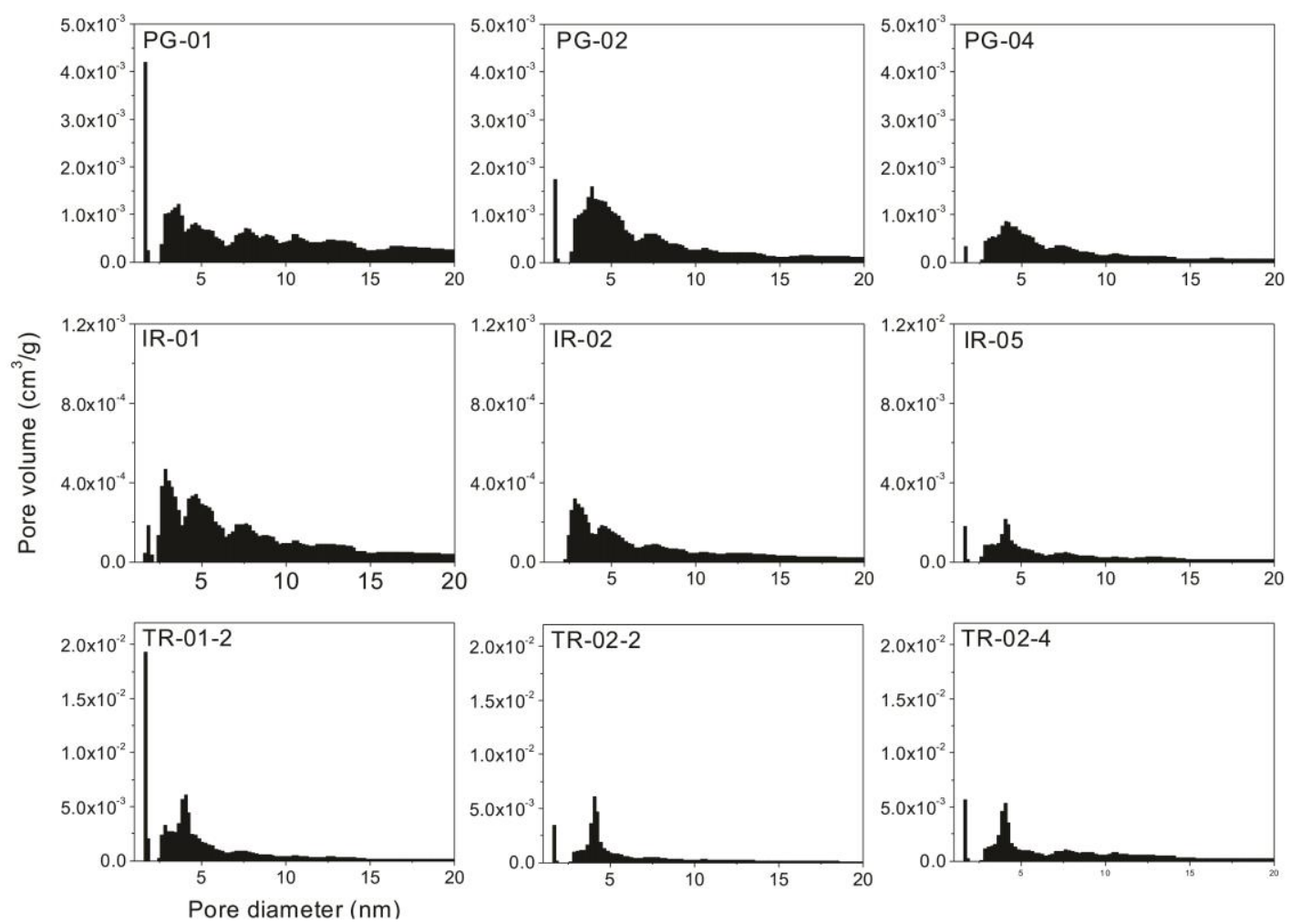

Figure 7 - PSD histograms of nine samples representing average patterns of each studied unit. Evaluation of pore size distribution from DFT analysis demonstrates wider distribution for Ponta Grossa and Irati samples than for Tremembé samples. 

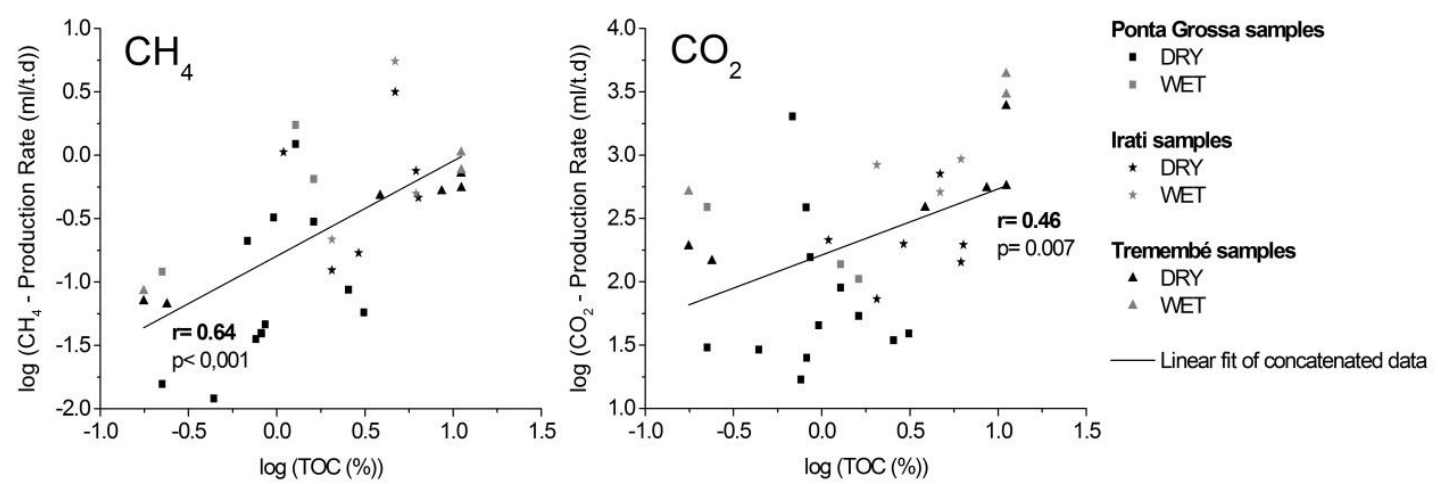

Figure 8 - Comparison between $\mathrm{CH}_{4}$ and $\mathrm{CO}_{2}$ production and TOC for dry (DRY) and wet (WET) samples tests. Linear regression and correlation coefficient is showed for grouped data.

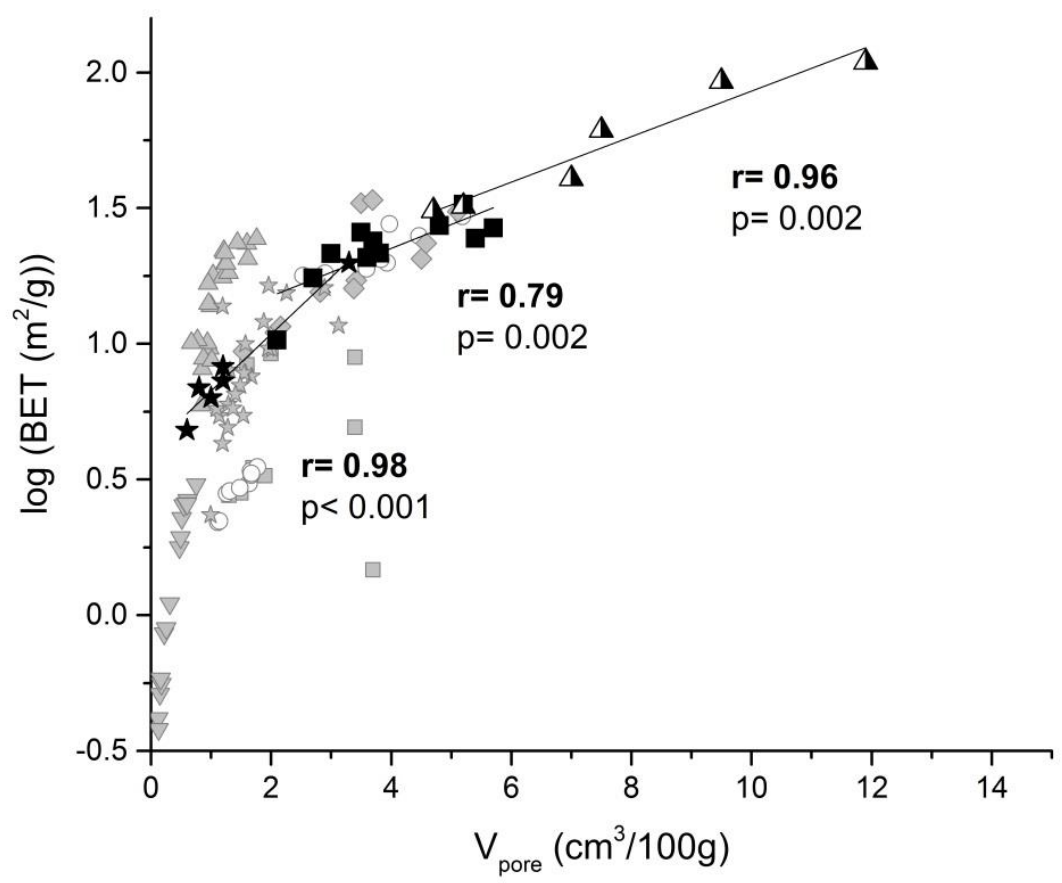

Figure 9 - Comparison between nitrogen adsorption results obtained in this study (black symbols) and in previous studies from the literature (gray symbols). Positive linear correlation is observed between pore volume $\left(\mathrm{V}_{\text {pore }}\right)$ and $\mathrm{S}_{\text {area }}$ for Ponta Grossa (black squares), Irati (black stars) and Tremembé (black triangles) samples. Data from previous studied obtained from organic rich shales of Lower Yangtze Region (up triangles; Pan et al., 2015), Ordos Basin (squares; Ji et al., 2014 and down triangles; Liu et al., 2015), Sichuan Basin (diamonds; Yang et al., 2014 and open circles; Cao et al., 2015), Perth Basin and Canning Basin (stars; Labani et al., 2013). 
Table 1 - Location of the outcrops of Ponta Grossa (12 samples), Irati (6 samples) and Tremembé (6 samples) Formations.

\begin{tabular}{|c|c|c|c|c|}
\hline Sampling site & Formation & № of samples & Latitude & Longitude \\
\hline PG-01 & Ponta Grossa & 1 & $24^{\circ} 4^{\prime} 47^{\prime \prime S}$ & $49^{\circ} 46^{\prime} 30^{\prime \prime W}$ \\
\hline PG-02 & Ponta Grossa & 1 & $24^{\circ} 4^{\prime} 48^{\prime \prime S}$ & $49^{\circ} 46^{\prime} 24^{\prime \prime W}$ \\
\hline PG-03 & Ponta Grossa & 4 & $24^{\circ} 14^{\prime} 30^{\prime \prime S}$ & $49^{\circ} 43^{\prime} 55^{\prime \prime} \mathrm{W}$ \\
\hline PG-04 & Ponta Grossa & 1 & $24^{\circ} 14^{\prime} 24^{\prime \prime S}$ & $49^{\circ} 43^{\prime} 57^{\prime \prime} \mathrm{W}$ \\
\hline PG-05 & Ponta Grossa & 1 & $24^{\circ} 14^{\prime} 24^{\prime \prime S}$ & $49^{\circ} 43^{\prime} 57^{\prime \prime} \mathrm{W}$ \\
\hline PG-06 & Ponta Grossa & 4 & $24^{\circ} 30^{\prime} 03^{\prime \prime S}$ & $50^{\circ} 26^{\prime} 45^{\prime \prime} \mathrm{W}$ \\
\hline IR-01 & Irati & 1 & $25^{\circ} 53^{\prime} 35^{\prime \prime S}$ & $50^{\circ} 22^{\prime} 42^{\prime \prime} \mathrm{W}$ \\
\hline IR-02 & Irati & 1 & $25^{\circ} 49^{\prime} 34^{\prime \prime S}$ & $50^{\circ} 24^{\prime} 52 " \mathrm{~W}$ \\
\hline IR-03 & Irati & 2 & $22^{\circ} 32^{\prime} 41^{\prime \prime S}$ & $47^{\circ} 26^{\prime} 26^{\prime \prime} \mathrm{W}$ \\
\hline IR-04 & Irati & 1 & $23^{\circ} 10^{\prime} 16^{\prime \prime S}$ & $47^{\circ} 57^{\prime} 06^{\prime \prime} \mathrm{W}$ \\
\hline IR-05 & Irati & 1 & $25^{\circ} 47^{\prime} 12^{\prime \prime S}$ & $50^{\circ} 25^{\prime} 58^{\prime \prime} \mathrm{W}$ \\
\hline TR-01 & Tremembé & 2 & $23^{\circ} 00^{\prime} 15^{\prime \prime S}$ & $45^{\circ} 35^{\prime} 05^{\prime \prime} \mathrm{W}$ \\
\hline TR-02 & Tremembé & 4 & $22^{\circ} 57^{\prime} 01^{\prime \prime S}$ & $45^{\circ} 32^{\prime} 26^{\prime \prime} \mathrm{W}$ \\
\hline
\end{tabular}

Table 2 - Means $(\bar{x})$, minima $(\min )$, maxima $(\max )$ and standard deviations $(\sigma)$ of the obtained values of total organic carbon (TOC) and biogenic gas production rates (PR) for dry sample tests (DRY), headspace refilling (R), acetic acid addition (AA) and wet sample tests (WET) from Ponta Grossa, Irati and Tremembé samples

\begin{tabular}{|c|c|c|c|c|c|c|c|c|c|c|}
\hline \multirow{3}{*}{\multicolumn{2}{|c|}{$\begin{array}{l}\text { Geologic } \\
\text { Formation }\end{array}$}} & \multirow{3}{*}{$\begin{array}{c}\text { TOC } \\
\% \\
\end{array}$} & \multicolumn{2}{|c|}{ DRY PR } & \multicolumn{2}{|c|}{ R PR } & \multicolumn{2}{|c|}{ AA PR } & \multicolumn{2}{|c|}{ WET PR } \\
\hline & & & $\mathrm{CH}_{4}$ & $\mathrm{CO}_{2}$ & $\mathrm{CH}_{4}$ & $\mathrm{CO}_{2}$ & $\mathrm{CH}_{4}$ & $\mathrm{CO}_{2}$ & $\mathrm{CH}_{4}$ & $\mathrm{CO}_{2}$ \\
\hline & & & \multicolumn{8}{|c|}{$\mathrm{ml} / \mathrm{t} . \mathrm{d}$} \\
\hline \multirow{4}{*}{$\begin{array}{l}\text { Ponta } \\
\text { Grossa }\end{array}$} & $\overline{\mathbf{x}}$ & 1.18 & 0.20 & 244.00 & 0.00 & 19.53 & 0.17 & 840.65 & 0.84 & 211.07 \\
\hline & $\sigma$ & 0.82 & 0.33 & 544.94 & 0.00 & 27.62 & 0.12 & 352.57 & 0.67 & 126.91 \\
\hline & $\min$ & 0.22 & 0.01 & 16.93 & 0.00 & 0.00 & 0.03 & 437.86 & 0.12 & 105.61 \\
\hline & $\max$ & 3.11 & 1.23 & 2021.43 & 0.00 & 58.59 & 0.32 & 1296.56 & 1.74 & 389.57 \\
\hline \multirow{4}{*}{ Irati } & $\overline{\mathbf{x}}$ & 3.87 & 0.96 & 256.19 & 0.19 & 38.56 & 1.35 & 1836.60 & 2.08 & 758.97 \\
\hline & $\sigma$ & 2.00 & 1.04 & 209.46 & 0.14 & 54.53 & 1.27 & 683.53 & 2.44 & 179.64 \\
\hline & $\min$ & 1.09 & 0.12 & 73.11 & 0.00 & 0.00 & 0.39 & 1234.19 & 0.22 & 510.60 \\
\hline & $\max$ & 6.34 & 3.17 & 712.46 & 0.35 & 115.68 & 3.15 & 2792.51 & 5.53 & 929.43 \\
\hline \multirow{4}{*}{ Tremembé } & $\overline{\mathbf{x}}$ & 5.84 & 0.40 & 716.27 & 0.07 & 1418.82 & 2.10 & 3974.15 & 0.64 & 2644.80 \\
\hline & $\sigma$ & 4.66 & 0.25 & 792.25 & 0.10 & 1087.88 & 1.89 & 2701.03 & 0.41 & 1606.74 \\
\hline & $\min$ & 0.18 & 0.07 & 146.58 & 0.00 & 293.98 & 0.05 & 854.57 & 0.09 & 516.52 \\
\hline & $\max$ & 11.11 & 0.72 & 2450.80 & 0.21 & 2890.24 & 4.61 & 7442.99 & 1.05 & 4398.32 \\
\hline
\end{tabular}


Table 3 - Pore structure parameters of shale samples from Ponta Grossa, Irati and Tremembé Formations. Besides $S_{\text {area }}, V_{\text {pore }}$ and $\mathrm{M}_{\text {ratio }}$, average pore diameter $\left(D_{\text {pore }}\right)$, micropore volume $\left(\mathrm{V}_{\text {micropore }} \mathrm{DFT}\right)$ and pore volume calculated by DFT ( $\left.\mathrm{V}_{\text {total }} \mathrm{DFT}\right)$ are also presented.

\begin{tabular}{|l|c|c|c|c|c|c|c|}
\hline \multicolumn{1}{|c|}{ Sample } & $\begin{array}{c}\mathbf{S}_{\text {area }} \\
\mathbf{B E T}\left(\mathrm{m}^{2} / \mathrm{g}\right)\end{array}$ & $\begin{array}{c}\mathbf{D}_{\text {pore }} \\
\mathbf{B J H} \\
(\mathrm{nm})\end{array}$ & $\begin{array}{c}\mathbf{D}_{\text {pore }} \\
\mathbf{D F T} \\
(\mathrm{nm})\end{array}$ & $\begin{array}{c}\mathbf{V}_{\text {pore }} \\
\left(\mathrm{cm}^{3 / 100} \mathrm{~g}\right)\end{array}$ & $\begin{array}{c}\mathbf{V}_{\text {micropore }} \mathbf{D F T} \\
\left(\mathrm{cm}^{3 / 100} \mathrm{~g}\right)\end{array}$ & $\begin{array}{c}\mathbf{V}_{\text {total }} \\
\text { DFT } \\
\left(\mathrm{cm}^{3 / 100} \mathrm{~g}\right)\end{array}$ & $\begin{array}{c}\mathbf{M}_{\text {ratio }} \\
\text { DFT } \\
(\%)\end{array}$ \\
\hline PG-01 & 27 & 3.35 & 3.63 & 5.49 & 0.44 & 4.8 & 9.2 \\
\hline PG-02 & 22 & 3.35 & 3.79 & 4.21 & 0.18 & 3.8 & 4.7 \\
\hline PG-03-1 & 21 & 3.37 & 3.79 & 4.04 & 0.19 & 3.6 & 5.4 \\
\hline PG-03-2 & 21 & 3.35 & 3.79 & 3.33 & 0.27 & 3 & 8.8 \\
\hline PG-03-3 & 23 & 3.36 & 3.79 & 4.17 & 0.31 & 3.7 & 8.4 \\
\hline PG-03-4 & 26 & 3.36 & 3.79 & 3.96 & 0.38 & 3.5 & 10.8 \\
\hline PG-04 & 10 & 3.4 & 4.15 & 2.32 & 0.03 & 2.1 & 1.6 \\
\hline PG-05 & 33 & 3.38 & 3.79 & 5.81 & 0.52 & 5.2 & 10.0 \\
\hline PG-06-1 & 24 & 3.35 & 3.63 & 4.12 & 0.39 & 3.7 & 10.6 \\
\hline PG-06-2 & 17 & 3.04 & 3.79 & 3.02 & 0.19 & 2.7 & 6.9 \\
\hline PG-06-3 & 27 & 3.36 & 3.63 & 6.59 & 0.24 & 5.7 & 4.3 \\
\hline PG-06-4 & 24 & 3.37 & 4.75 & 6.00 & 0.19 & 5.4 & 3.5 \\
\hline IR-01 & 7 & 3.04 & 2.77 & 1.37 & 0.03 & 1.2 & 2.2 \\
\hline IR-02 & 5 & 3.09 & 2.77 & 0.76 & 0.00 & 0.6 & 0.0 \\
\hline IR-03-1 & 6 & 3.05 & 4.15 & 1.15 & 0.06 & 1 & 6.4 \\
\hline IR-03-2 & 7 & 3.05 & 2.77 & 0.96 & 0.13 & 0.8 & 15.7 \\
\hline IR-04 & 8 & 3.38 & 4.15 & 1.41 & 0.09 & 1.2 & 7.7 \\
\hline IR-05 & 20 & 3.4 & 4.15 & 3.69 & 0.19 & 3.3 & 5.7 \\
\hline TR-01-1 & 109 & 3.35 & 4.15 & 12.9 & 2.24 & 11.9 & 18.8 \\
\hline TR-01-2 & 93 & 3.34 & 4.15 & 10.3 & 2.13 & 9.5 & 22.4 \\
\hline TR-02-1 & 61 & 3.34 & 4.34 & 8.15 & 1.21 & 7.5 & 16.1 \\
\hline TR-02-2 & 31 & 3.34 & 4.15 & 5.09 & 0.35 & 4.7 & 7.5 \\
\hline TR-02-3 & 32 & 3.34 & 4.15 & 5.69 & 0.31 & 5.2 & 6.0 \\
\hline TR-02-4 & 41 & 3.36 & 3.97 & 7.64 & 0.58 & 7 & 8.3 \\
\hline
\end{tabular}

